\title{
Methods to improve the sample quality of macromolecular complexes for structure determination by 3D Electron Cryo-Microscopy
}

\author{
$\mathrm{D}$ is se r t a $\mathrm{t}$ io $\mathrm{n}$ \\ zur Erlangung des mathematisch-naturwissenschaftlichen \\ Doktorgrades \\ "Doctor rerum naturalium" \\ der Georg-August-Universität Göttingen \\ vorgelegt von \\ Florian Peter Platzmann \\ aus Nürnberg
}




\section{Mitglieder des Betreuungssausschusses:}

Prof. Dr. Holger Stark (Referent)

Prof. Dr. Reinhard Lührmann (Koreferent)

Prof. Dr. Ralf Ficner

Tag der mündlichen Prüfung: ～24. Februar 2012 


\section{Affidavit}

Hiermit erkläre ich an Eides statt, dass ich die vorliegende Arbeit selbständig angefertigt habe und nur die angegebenen Hilfsmittel verwendet wurden. 

Für meine Eltern, die mir stets Wurzeln und Flügel gaben

und für Dr. Volker Thorn, der mich dazu inspirierte,

Chemie zu studieren. 


\section{List of Publications}

Z.Warkocki, P. Odenwälder, J. Schmitzová, F. Platzmann, H. Stark, H. Urlaub, R. Ficner, P. Fabrizio, and R. Lührmann. Reconstitution of both steps of saccharomyces cerevisiae splicing with purified spliceosomal components. Nat Struct Mol Biol, 16(12):1237-1243, 2009. 


\section{Contents}

$\begin{array}{ll}\text { Abstract } & 1\end{array}$

1 Introduction 3

1.1 Transmission Electron Microscopy . . . . . . . . . . 3

1.1.1 Image Processing in single-particle EM $\ldots \ldots$

1.1.2 General procedure ............. 8

1.1.3 Angular Reconstitution . . . . . . . . . . 8

1.1.4 Projection matching $\ldots \ldots \ldots . \ldots 9$

1.1.5 Supervised classification . . . . . . . . . 11

1.2 Sample heterogeneity . . . . . . . . . . . . 11

1.2.1 Structural and conformational heterogeneity . . . . 12

1.2.2 The GraFix preparation protocol . . . . . . . . . 13

1.2.3 Macromolecular Crystallization additives . . . . 16

1.3 Macromolecular complexes . . . . . . . . . . 16

1.3.1 The spliceosome $\ldots \ldots \ldots 17$

1.3.2 The ribosome . . . . . . . . . . . . . 19

1.3.3 Glutamate dehydrogenase .......... 20 
1.4 Aim of the work . . . . . . . . . . . . . . . . . 21

2 Materials \& Methods $\quad 23$

2.1 Materials ..................... 23

2.1 .1 Chemicals ................. 23

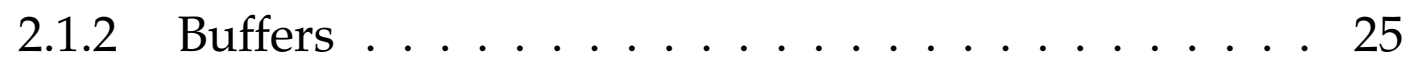

2.1.3 Laboratory materials . . . . . . . . . 26

2.1.4 Special Equipment ............. 26

2.1 .5 Software ................... 27

2.2 Biochemical Methods . . . . . . . . . . . . . . . 28

2.2.1 Assembly and purification of the human spliceosomal B-complex . . . . . . . . . . . . . 28

2.2.2 Polyacrylamide gel electrophoresis . . . . . . 28

2.3 GraFix sample-preparation . . . . . . . . . . 28

2.3.1 Spliceosomal remodeling . . . . . . . . . . 29

2.3.2 Chemical modification . . . . . . . . . . 29

2.3.3 Cryo-Preparation .............. 30

2.3.4 Glutamate dehydrogenase .......... . 30

2.3 .570 ribosome $\ldots \ldots \ldots \ldots$

2.4 Sample preparation for single-particle EM . . . . . . . 31

2.4.1 Preparation of carbon film ......... . 31

2.4.2 Preparation of EM-grids coated with holey carbon film ...................... 31

2.4.3 Preparation of negative stain samples . . . . . . 32 
2.4.4 Preparation of unstained cryo-samples . . . . . . 33

2.5 Electron microscopy analysis . . . . . . . . . 33

2.5.1 Transmission electron microscopy . . . . . . . . 33

2.5.2 Raw image processing . . . . . . . . . . 34

2.5 .3 Image Processing . . . . . . . . . . . . . 34

3 Results $\quad 35$

3.1 Spliceosomal remodeling EM-studies . . . . . . . . . 35

3.2 Chemical modification . . . . . . . . . . . 38

3.2.1 GraFix Preparation . . . . . . . . . . . . 38

3.2.2 Image aquisition $\ldots \ldots . \ldots . \ldots 39$

3.2.3 Image Processing . . . . . . . . . . . . . . 39

3.2.4 Fourier Ring Correlation . . . . . . . . . . . . . 43

3.3 Cryo-Fixation .................... 46

3.3.1 Adapting the GraFix protocol for cryo-fixation . . . 46

3.3.2 Validation with the $70 \mathrm{~S}$ ribosome . . . . . . 49

4 Discussion $\quad 57$

4.1 Spliceosomal remodeling EM-studies . . . . . . . . . 57

4.1 .1 Outlook .................... 58

4.2 Chemical modification . . . . . . . . . . 58

4.2 .1 Image level $\ldots \ldots . \ldots 59$

4.2.2 FRC-analysis . . . . . . . . . . . . . 59

4.2 .3 Outlook ..................... 59 


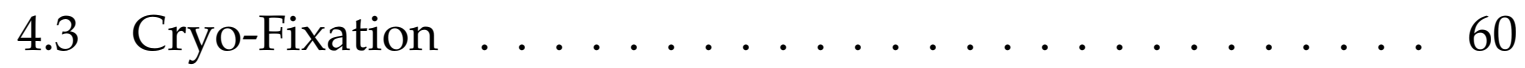

4.3.1 The CryoFix protocol . . . . . . . . . . . 61

4.3.2 The ribosome as a model system . . . . . . . . 61

4.4 Conclusion and Outlook . . . . . . . . . . . . . 63

Abbreviations

Bibliography

66

Acknowledgements 
I am among those who think that science has great beauty. A scientist in his laboratory is not only a technician: he is also a child placed before natural phenomena which impress him like a fairy tale.

- Marie Curie (1867 - 1934) 



\section{Abstract}

Macromolecular assemblies often undergo substantial structural rearrangements. Not only do functional states differ from each other, a single functional state also exhibits inherent conformational heterogeneity. A typical example is the yeast spliceosomal B-complex before and after catalytic activation by Prp2.

Electron microscopy datasets of both states were compared and it could be verified that the complex undergoes a significant structural remodelling upon activation. Conformational heterogeneity limits the resolution of the 3D electron microscopy reconstruction or necessitates the solution of several distinct substructures - an often time consuming process that also requires large datasets. For many cases, reduction of conformational freedom within the sample would be advantageous: More structural information could be gained from a smaller dataset.

The GraFix preparation protocol significantly improves the sample quality of macromolecules by stabilizing the sample chemically with glutaraldehyde, a crosslinking reagent, in a density gradient. Both chemical and physical modifications of the protocol were tested. Several compounds were added to the gradient and their effects on heterogeneity were evaluated on the 2D-level. However, the modifications did not lead to a definitive improvement in sample quality. Efforts were refocused on the temperature at which the sample is exposed to the crosslinking reagent. It was evaluated whether a fixation at temperatures below $0{ }^{\circ} \mathrm{C}$ would affect the sample to adopt a thermodynamically favoured conformation and basically "freeze" movement within the structure. To account for reduced glutaraldehyde activity at lower temperatures, the fixation conditions were optimized using glutamate dehydrogenase as a model assembly. As a proof of principle, $70 S$ ribosome samples were subjected to cryo-fixation. Their distribution of intersubunit rotation angles was compared to that of data recorded with standard-GraFix-prepared samples as well as unfixated samples. The cryo-fixated samples showed a noticeably higher homogeneity in their conformational distribution. 
Abstract

It was proven that glutaraldehyde fixation can stabilize a conformational state at lower exposure temperature and retain it even upon subsequent warming to higher preparation temperatures. Thus, the "CryoFix protocol" was established, which further limits heterogeneity. This is one more step towards higher quality EM-structures. 


\section{Introduction}

For scientific understanding, visualization has always been crucial. Diagrams, charts and graphs help to understand vast columns of numbers. Abstract topics can often be reduced to simple concepts with the right illustration. And it is one thing to have a complex mechanism explained and supported by numeric data - but it is a completely different thing to see it come to life in the form of an image or, in recent times, as an animation. Even before the processes inside the cell could be accurately portrayed, scientists tried to describe them, often with abstract but sometimes surprisingly accurate approximations. But to fully understand the processes that govern life, where form and function are tightly intertwined, visual understanding plays a key role.

Through techniques like cryo-electron microscopy (cryo-EM) or X-ray crystallography we are able to look inside the cells and see the very building blocks of life - not with our own eyes but with the help of devices that correctly depict their shape and let us glimpse even the smallest details. Armed with this knowledge, we might able to comprehend the fundamental processes that constitute the molecular basis of live. This work is a small step in that venture: By decreasing structural heterogeneity in cryo-EM samples more details may become visible, and the resolution boundary for imaging macromolecular assemblies is pushed a little further.

\subsection{Transmission Electron Microscopy}

Since ancient times, lenses have been used to magnify small objects and study them. From glass globes filled with water to the intricate array of precision lenses in compound microscopes, optical magnification has come a long way and allowed ever more detailed insight into the microscopic world. But even with perfect lenses, classical light microscopy reaches its resolution limit - i.e. the distance at which two neighbouring features cannot be clearly distinguished from 


\section{Introduction}

each other - at a resolution of about $200 \mathrm{~nm}$. This diffraction limit is named after Ernst Karl Abbe, who discovered in 1873 that the resolvable feature size $d$ is dependent on the wavelength $\lambda$ and the numerical aperture $N A$, which contains the refractive index $n$ of the medium the light travels through and $\vartheta$, the half-angle of the maximum cone of light that can enter or exit the lens.

$$
d=\frac{\lambda}{2 \cdot N A}=\frac{\lambda}{2 \cdot(n \cdot \sin \vartheta)}
$$

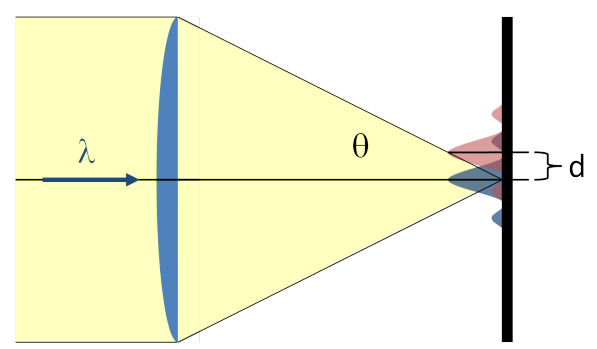

Figure 1.1: Visualization of the Abbe diffraction limit.

Louis-Victor de Broglie postulated the wave character of electrons in 1924 according to the formula

$$
\lambda=\frac{h}{p}
$$

where $h$ is Planck's constant and $p$ the momentum of the particle. Since electrons have a significantly smaller wavelength than visible light (depending on the velocity of the electrons, but below $0.01 \mathrm{~nm}$ ), the feature size that could be resolved with electrons is equally smaller. Resolution would no longer be limited by the wavelength but by the quality of the lens.

The first transmission electron microscope (TEM) was built in 1931 by Ernst Ruska (19061988, Nobel Prize in Physics in 1986) and had a magnification of only 17x - comparable to a magnifying glass. It proved, however, that an electron beam can be refracted and focussed with electromagnetic lenses as light with conventional lenses. In 1933 the Abbe limit was surpassed when another prototype reached a magnification of 12,000x. With significant improvements in the following years, the magnification and quality of electron microscopic images could be further increased. Modern TEMs achieve a resolution below $0.1 \mathrm{~nm}$ and can thus resolve atomic details, for example in materials science. Biological samples cannot sustain the high electron beam energy required to achieve atomic resolution and would decompose in the electron beam. 


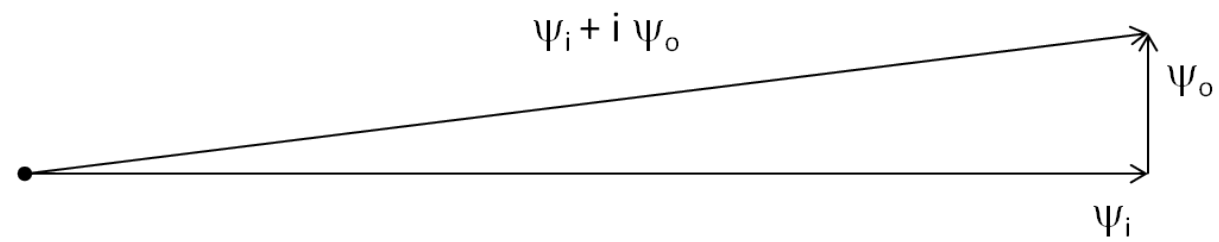

a) No phase contrast

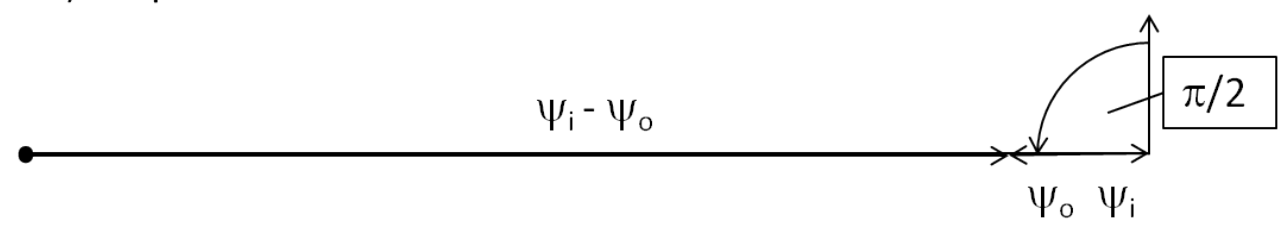

b) Positive phase contrast

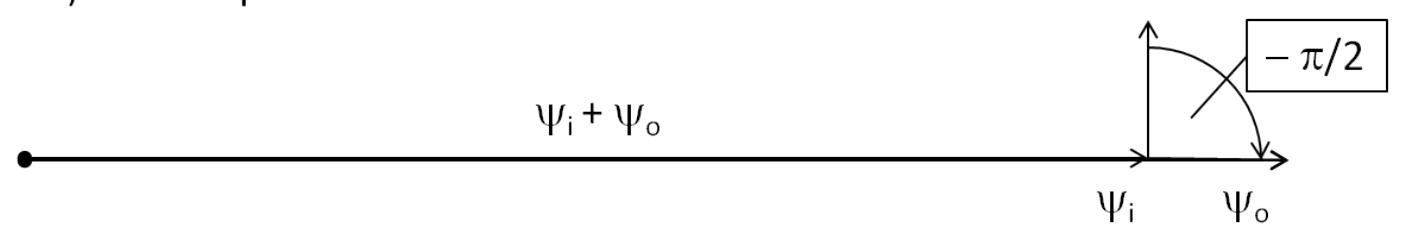

c) Negative phase contrast

Figure 1.2: (a) Vector addition of the image amplitude $\Psi_{i}$ and the scattered amplitude $\Psi_{o}$ phase shifted by $\pi / 2$ or $90^{\circ}$, (b) positive phase contrast produced by an additional phase shift of $+\pi / 2$, (c) negative phase contrast produced by an additional phase shift of $-\pi / 2$ or $+3 \pi / 2$

Image contrast in TEM comes from two sources: Amplitude contrast and phase contrast. The former is caused by electrons being inelastically scattered into the aperture or absorbed by the sample, which thus directly decreases the intensity of the electron beam. With the thin samples used in biological TEM, amplitude contrast plays only a minor role, since the light nuclei of biomolecules (mainly $\mathrm{C}, \mathrm{H}, \mathrm{O}, \mathrm{N}, \mathrm{S}, \mathrm{P}$ ) scatter only weakly. However, the absorbed electrons still deposit energy on the sample and cause radiation damage, effectively limiting the beam intensity that can be employed without damaging the specimen too much while the images are taken [Henderson, 1995]. Electron microscopy of biological macromolecules is therefore performed under "low-dose" conditions of $<30 e^{-} / \AA^{2}$. This results in blurry images with low signal-to-noise ratio and necessitates image processing methods to obtain the desired details. Phase contrast accounts for the majority of the image contrast in electron microscopy of biomolecules. It is caused by an interaction of electrons in the Coulomb field of the atomic nucleus. The emerging object wave has its phase shifted compared to the undiffracted initial wave. The resulting phase contrast is almost invisible in the focal plane. It can be visualized by defocusing the objective lens and thereby introducing an additional phase shift through the 


\section{Introduction}

spherical aberration $C_{s}$ of the electron lens, so that the amplitudes of the initial wave and the object wave produce a constructive interference. By varying the defocus the phase contrast can be modified. The optimal defocus with a maximized positive phase contrast was discovered to be a very weak underfocus of $\left(\sqrt{C_{S} \lambda}\right)$ and is termed "Scherzer focus" after its discoverer Otto Scherzer [Reimer, 1997]. Typically, larger defoci are used for biological specimen.

\subsubsection{Image Processing in single-particle EM}

The images obtained from TEM are two-dimensional projections of three-dimensional objects. During preparation, the sample is plunged into liquid ethane and rapidly cooled down [Adrian et al., 1984]. The high cooling rate leads to vitrification of the water, where it freezes without forming ice crystals [Brüggeller and Mayer, 1980]. The sample particles are immobilized and encased in a thin layer of vitreous ice where they are randomly distributed with regard to translation and rotation. Alternatively, the sample can be prepared at room temperature with the negative stain method [Leberman, 1965]. Here, the sample is exposed to a $2 \%$ uranyl formate solution for about 2 minutes (for a detailed description, see section 2.4 .3 on page 32 ). The heavy metal salt (the "stain") adsorbs to the particles and the grid surface. The heavy atoms of the stain increase the contribution of amplitude contrast, which is clearly visible, and allow the imaging of "protein shaped holes" in the salt (hence the term "negative" stain).

The projected single-particle images are extracted from digital micrographs and processed as a dataset from which one or more three-dimensional structures can be reconstructed. For image analysis in Fourier space, the images are band-pass-filtered and normalized. By this, very low spatial frequencies are removed, which only represent image properties unrelated to the particle structure (e.g. varying ice thickness or stain artifacts). Very high spatial frequencies, which are susceptible to interference from noise, are also dampened. Additionally, a circular mask is applied to facilitate rotational alignment and reduce the information to mostly particle information. The dataset is then processed in several cycles on the 2D level before the actual 3D reconstruction. The general procedure for reconstruction employing "reference-free" alignment [Penczek et al., 1992] is presented in the following section. 


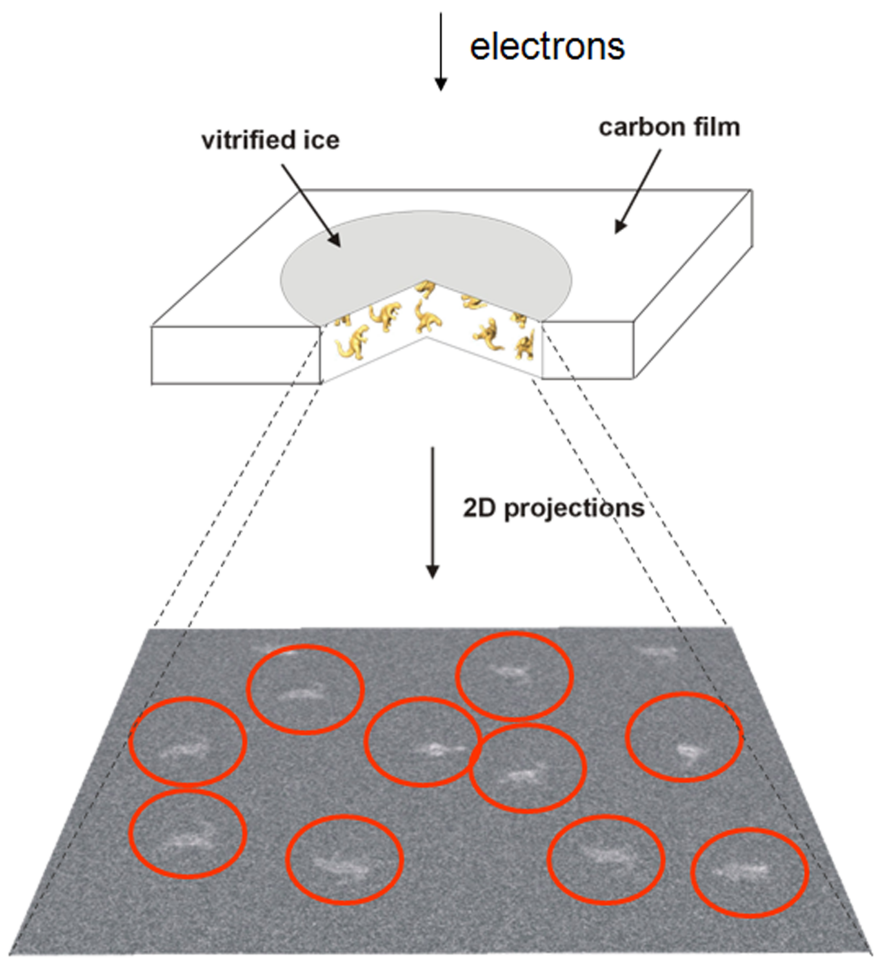

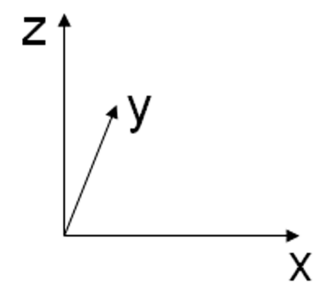

3 translational parameters

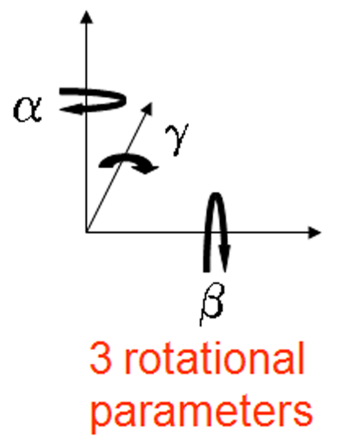

Figure 1.3: Left: 2D Image formation from 3D samples in electron microscopy. Right: Translational and rotational degrees of freedom. Image courtesy of Holger Stark.

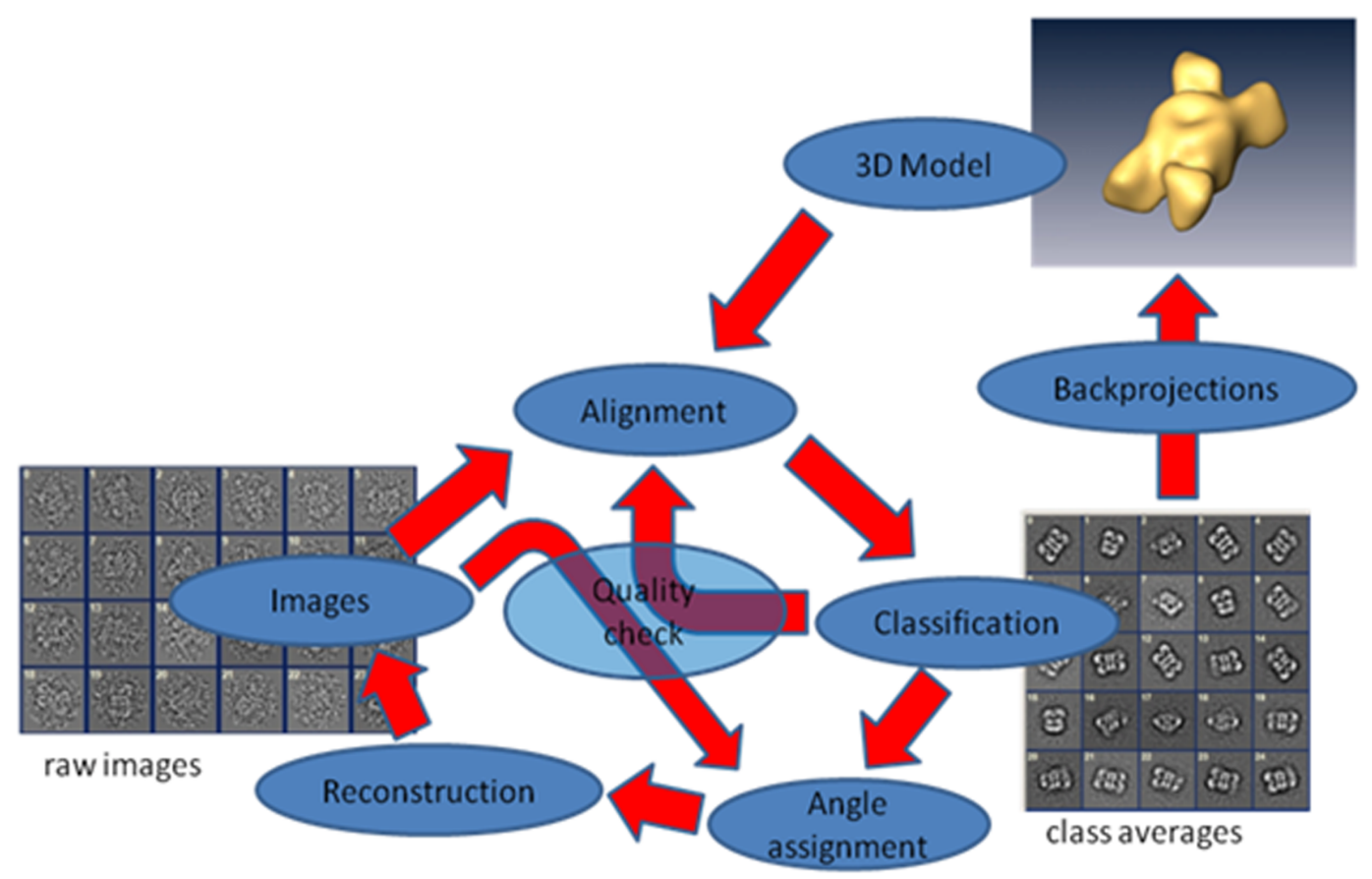

Figure 1.4: Refinement cycles in image processing. Filtered images are aligned to a set of references and classified via multivariate statistical analysis. After Euler angle assignment, a $3 \mathrm{D}$ reconstruction is possible from which new references can be projected. Image courtesy of Martin Schmeisser. 


\subsubsection{General procedure}

Due to the low-dose of electrons employed during recording, the images obtained by TEM show a poor contrast and a low signal-to-noise ratio (SNR). This ratio can be improved by averaging images of particles with identical two-dimensional projections in the same orientation, or "views". To reduce the number of random orientations, a translational and rotational alignment [Sander et al., 2003b] is applied along with subsequent multivariate statistical analysis and classification [van Heel and Frank, 1981, van Heel, 1984] to find identical projections that belong to the same class of views. First, a rotational average of the entire dataset is used as a reference to properly center the particles. Identical views are classified and summed into class averages, which can be used as references in further cycles of alignment and classification to improve the quality of the resulting class averages. After the angular relationships of the final class averages have been determined, a 3D structure can be backprojected. The result is a 3D volume data or "EM-map". This reconstruction serves as a source of reference for further refinement by gaining new 2D views from the 3D structure, which are again used in a cycle of alignment and classification to improve the quality of the 3D model.
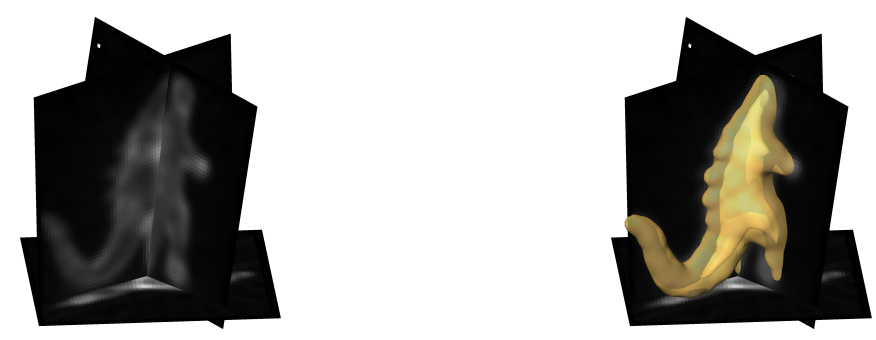

Figure 1.5: 2D projections arranged according to their angular relationship and the resulting 3D structure. Image courtesy of Florian Hauer.

\subsubsection{Angular Reconstitution}

In order to reconstruct a $3 \mathrm{D}$ volume from $2 \mathrm{D}$ projection images, the angular relations between the two-dimensional projections have to be determined. This is usually done via Euler angles, which describe the orientation in 3D space. The Euler convention uses three angles, $\alpha, \beta$ and $\gamma$, where $\alpha$ signifies a rotation around the $\mathrm{z}$-axis, followed by a rotation by $\beta$ around the new $\mathrm{x}$-axis and a final rotation by $\gamma$ around the $z$-axis resulting from the second step $\left(z, x, z^{\prime}\right)$. One method to 


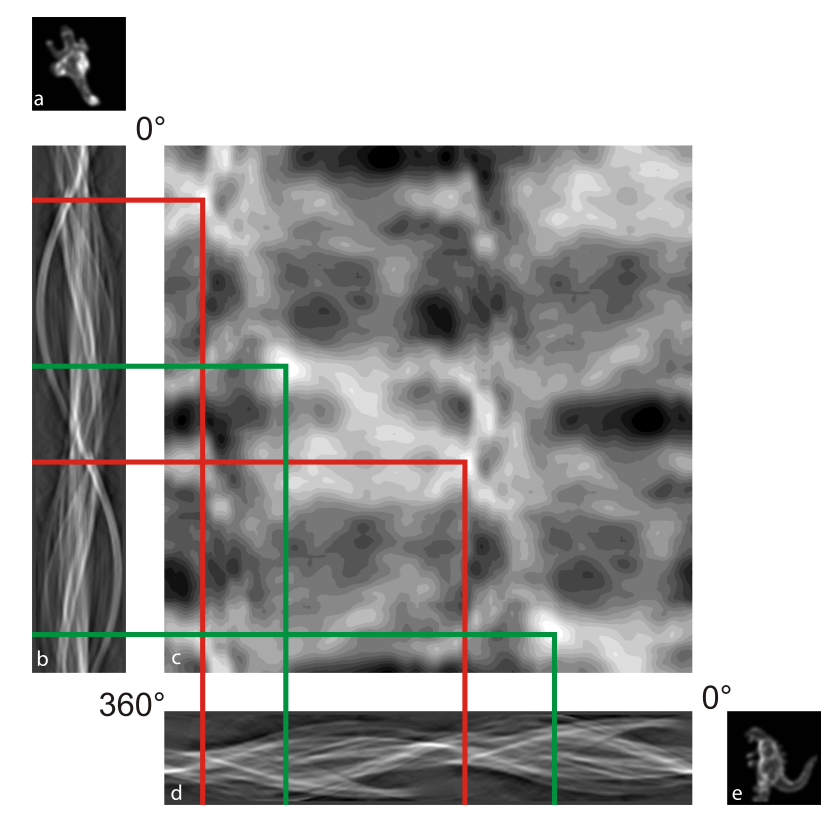

Figure 1.6: Angular reconstitution via the common line theorem. Two class averages $(\mathrm{a}, \mathrm{e})$ are projected into $1 \mathrm{D}$ lines which form sinograms $(b, d)$. The peaks in the sinogram correlation function (c) identify possible angular relations which have to be cerified by cross-referencing further images. Note the even number of peaks (with matching peaks being $180^{\circ}$ apart) due to an introduced symmetry during the sinogram projection. Image courtesy of Florian Hauer.

obtain these angles is angular reconstitution by employing the common line projection theorem. This utilises the assumption that two 2D projections of the same 3D object share at least one common 1D projection line [van Heel, 1987a]. In this method, two 2D images are projected separately to a $1 \mathrm{D}$ line, rotated by $1^{\circ}$ respectively and again projected. This is repeated over the full range of $360^{\circ}$ and all $1 \mathrm{D}$ projections of one image are appended below each other. The result is an image that, due to its sinoidal appearance, is called a sinogram. By correlating two sinograms line by line, a sinogram correlation function is obtained which can be displayed as a 2D greyscale map. The peaks of this map signify maximum correlation and the corresponding angles allow the determination of the angular relationship between the images.

\subsubsection{Projection matching}

If a reference 3D structure (e.g. from X-ray crystallography) already exists, initial angles can be be obtained by the so-called projection matching method [Penczek et al., 1994]. Instead of a reference-free reconstruction, the datasets are aligned to projections from an existing 3D structure. Since the angular relationships of these references are known from the projection 


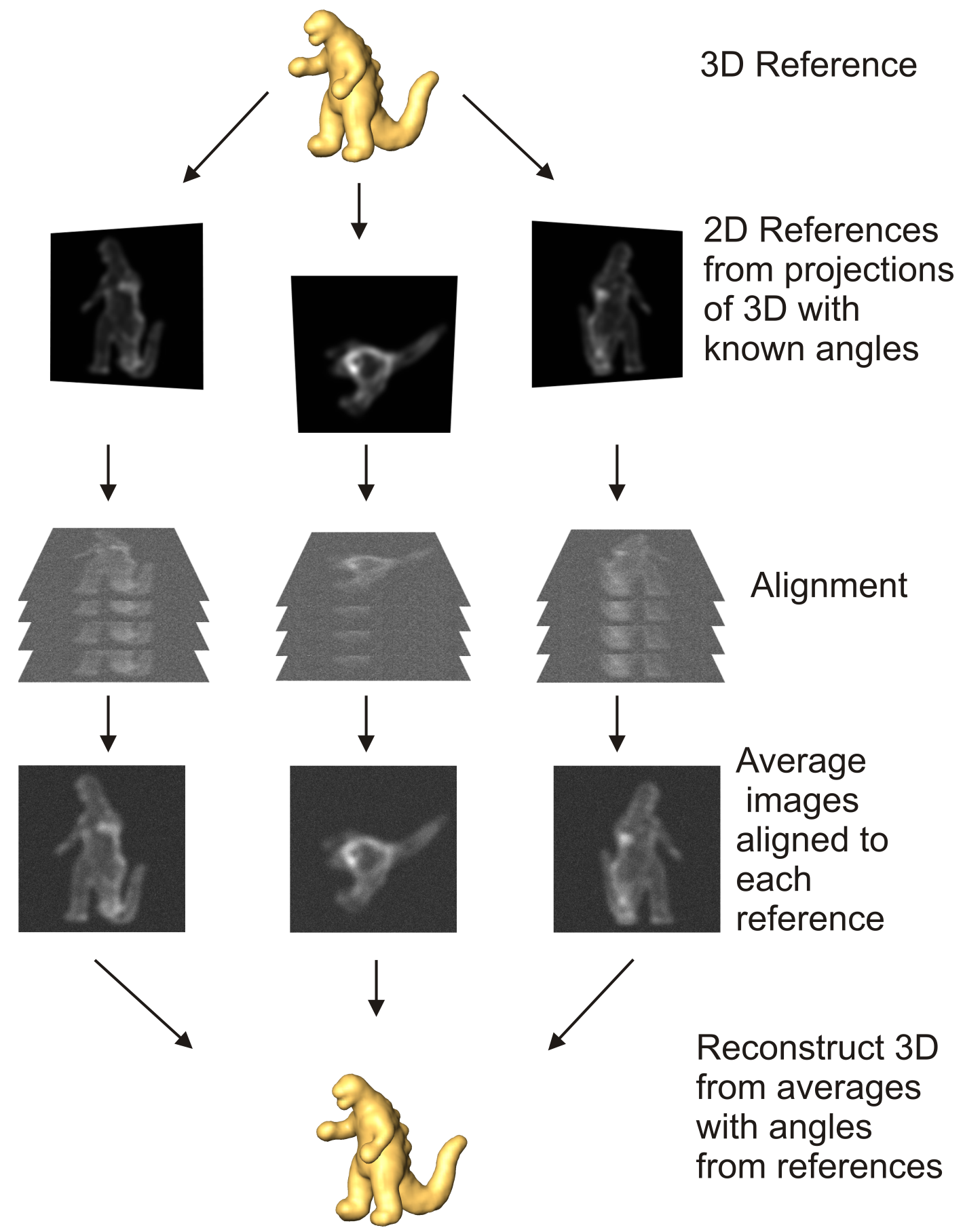

Figure 1.7: Schematic of projection matching. Image courtesy of Florian Hauer. 
source, this allows a direct 3D reconstruction. In single particle electron microscopy, projection matching is used to increase the resolution of the reconstruction once an initial model is available. By iteratively decreasing the angular distance of the projections, the theoretical resolution limit is decreased.

\subsubsection{Supervised classification}

In this work, the supervised classification method was used for the assessment of heterogeneity in the ribosome datasets [Gao et al., 2004]. In this method, a dataset to be evaluated is aligned to several sets of references projected from existing structures of different conformations. Each particle image is assigned to the reference to which it aligned with the best correlation. In this way, the dataset is divided into subsets, which can be individually refined, and the population of the respective heterogeneous states can be mapped according to this correlation.

\subsection{Sample heterogeneity}

The techniques described so far assume a homogeneous sample of identical particles. Images are averaged to improve the signal-to-noise ratio (SNR) of the noisy images and class averages are assigned angles to reconstruct a 3D map. However, with macromolecular assemblies, this ideal assumption is rarely the case. Often, several different functional states are observed or the complex even undergoes major structural rearrangements while exerting its function. Other complexes are assembled from several components and only exist for a short time in the desired state. In addition, the sample is subjected to a considerable amount of stress during biochemical purification and sample preparation, which can lead to particle damage and introduce heterogeneity. Several methods exist to assess and cope with sample heterogeneity in silico during image processing [Leschziner and Nogales, 2007]. Commonly, the heterogeneous features are represented on the 2D or 3D level in the form of several different references. These can be generated from the dataset itself or derived from existing structures, as for example in the aforementioned supervised classification. In all cases, the heterogeneous dataset is partitioned into homogeneous subsets, which are then treated independently.

In contrast, this work focuses on tackling the problem already in the preparation of the sample. 


\section{Introduction}

Reduced sample heterogeneity before image collection will simplify the subsequent image processing. To achieve optimal results, a combination of preparative and computational methods appears to be the most promising approach.

\subsubsection{Structural and conformational heterogeneity}

In electron microscopy, two kinds of heterogeneity have to be considered: Structural heterogeneity is caused by structurally different particles in the sample. These include impurities, oligomerized or degraded particles of the sample specimen. On the image level, it may be difficult to identify these unwanted particles from the residual population. If the images in the dataset are mixed, the resulting structure could be biased. Hence, to prevent structural heterogeneity, proper purification of the macromolecular assemblies is crucial. A preparation protocol named GraFix [Kastner et al., 2008], which is described in section 1.2.2, has proven to be particularly effective in preventing degradation of particles. Together with advanced purification methods it is very helpful in minimizing structural heterogeneity. Conformational heterogeneity on the other hand stems from particles that possess an inner dynamic or flexibility and can adopt different conformations. At the moment of vitrification, these particles may be present in any of their conformations, often only minimally different from each other. If not addressed properly, conformational heterogeneity leads to badly resolved reconstructions as the averaging of similar orientations but different conformations blurs the details in the dynamic region of the macromolecular complex. More often than not, the dynamic regions of macromolecules are functionally relevant and it is therefore of particular interest to reconstruct these regions with maximum detail.

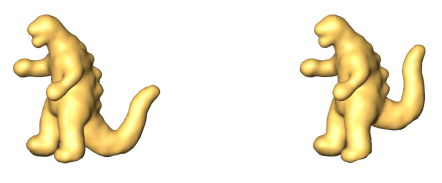

Heterogenous dataset

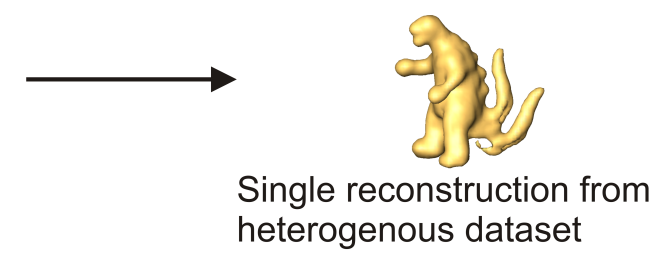

heterogenous dataset

Figure 1.8: Averaging of a conformationally heterogeneous dataset leads to falsified images. Image courtesy of Florian Hauer.

On the other hand, resolving heterogeneous datasets offers multiple advantages. Having different conformational states present in the sample allows visualization of conformational 
dynamics. This may help to better understand the function of a dynamic complex or even enable time-resolved analysis of biochemical processes, as has been successfully shown for the ribosome [Fischer et al., 2010]. However, this also involves a significantly higher workload. Several structures have to be reconstructed from the dataset, which has to be classified into one subset of images for each individual state. This leaves each conformation with only a fraction of the total recorded data contributing to its structure. To ensure sufficient detail and resolution, a correspondingly larger number of initial images has to be recorded and processed. In addition to that, there is the non-trivial task of identifying and assigning the respective conformational substructures. For this reason, conformational heterogeneity remains a challenge and methods to limit or even eliminate it would significantly enhance the possibility to achieve higher resolution in single-particle EM.

\subsubsection{The GraFix preparation protocol}

Biochemical protocols for preparation, isolation and purification of macromolecular complexes in biology are individually optimized; procedures vary greatly, as do conditions. The GraFix protocol (from Gradient Fixation), which has been established in our lab, is a universal final preparation step for single particle electron microscopy, and has been successfully used with a large number of samples [Kastner et al., 2008]. It combines a gradient sedimentation with a mild chemical fixation in a single centrifugation step. The sample is loaded on top of a gradient of increasing density and fixative and then centrifuged. This process not only stabilizes the particle but also serves as a final purification step. It is applicable to a wide variety of samples as well as buffer conditions. GraFix can be used for room temperature negative staining, for cryo negative stain and in non-stained cryo preparation. The schematics of the GraFix preparation are shown in Fig. 1.9 on the next page.

Fixation is typically accomplished with glutaraldehyde, a crosslinking reagent already known for electron microscopy preparation purposes but sometimes prone to generate artefacts [Hayat, 1986]. However, the combination of centrifugation and fixation offers many advantages compared to simply adding a fixation reagent directly to the sample. The particle encounters a slowly increasing concentration of the reagent, thus reducing the chance for inter-particle crosslinking. In addition, the pressure acting on the molecule during centrifugation dissolves weak, unspecific 


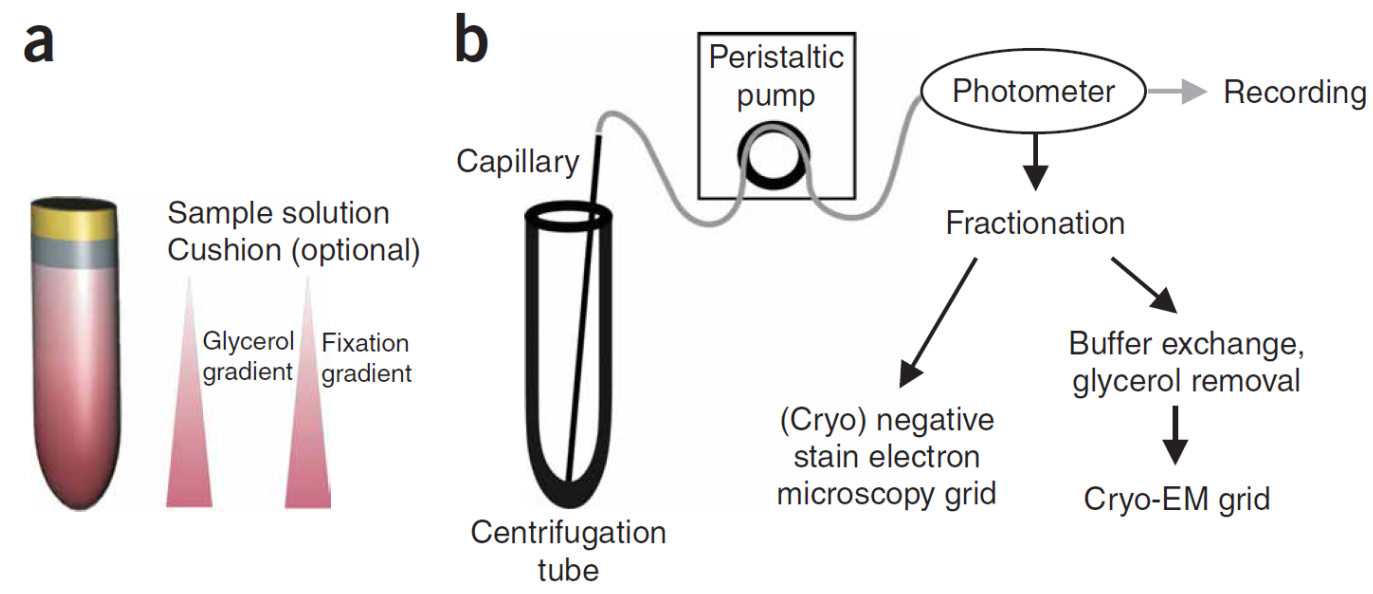

Figure 1.9: Schematics of the GraFix preparation. (a) The sample is applied on top of a density gradient and centrifuged. (b) Fractions are collected and the sample fraction is identified and further treated for EM-preparation. Image courtesy of Kastner et al. [2008].

crosslinking interactions that could otherwise lead to aggregate formation. The buffer conditions in the GraFix gradient can be customized for each individual sample. Buffer components which are incompatible with glutaraldehyde (e.g. Tris) can be exchanged with compatible ones (for example, HEPES). A small cushion of fixative-free buffer can be applied between the sample and the gradient upon loading to ease the transition. GraFix-treated samples show visibly reduced structural heterogeneity (i.e. less aggregated or degraded particles) and increased contrast in the electron microscope. This effect becomes even more prominent upon statistical image analysis. For example, the number of good-quality class averages in a sample of the spliceosomal B-complex increases about fivefold compared to an untreated sample and the signal is considerably higher due to increased structural homogeneity.

In addition, GraFix-treated samples also exhibit a higher stability and improved adsorption behaviour to the carbon support film during sample preparation (see section 2.4 on page 31). Without GraFix, standard adsorption times are generally limited to 1-2 minutes, because extended adsorption times can involve structural degradations of the particles. After GraFix treatment, adsorption time can not only be extended, but also offers a greater increase in the number of adsorbed particles over time compared to untreated samples. The increased stability of the particles is also useful during preparation of unstained cryo-grids. Since glycerol concentrations need to be very low in order to obtain a high contrast from macromolecular complexes embedded in vitrified ice during cryo-EM, the glycerol has to be removed. This is accomplished 
a

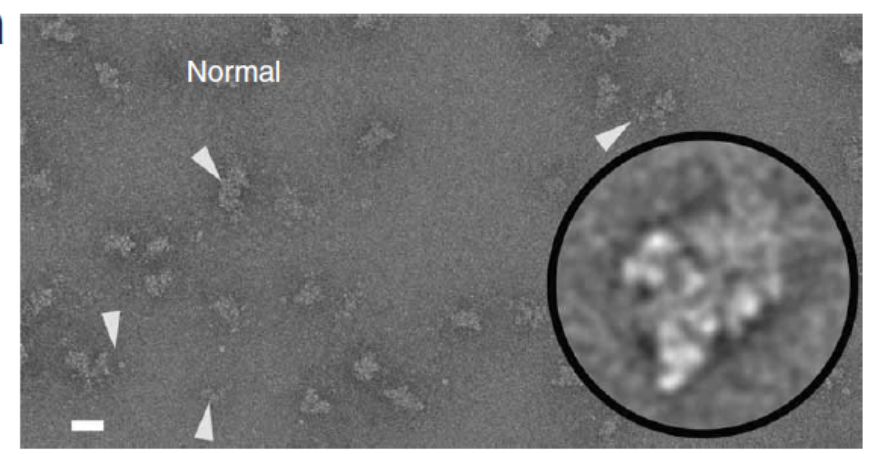

b

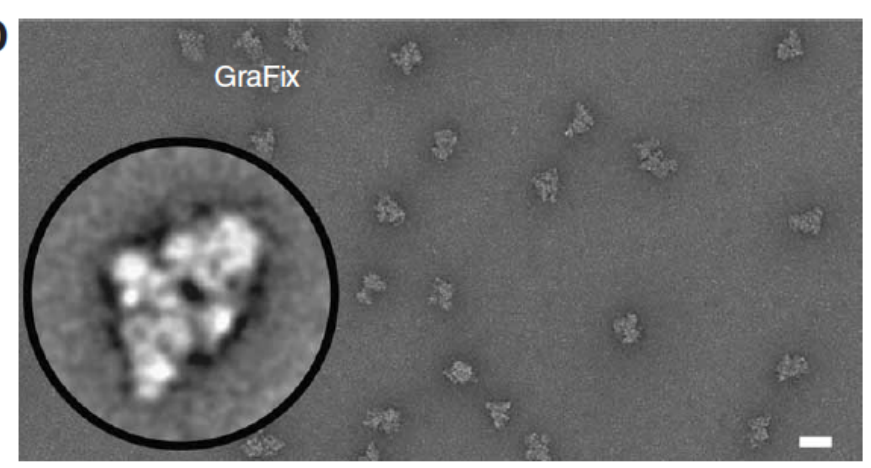

C

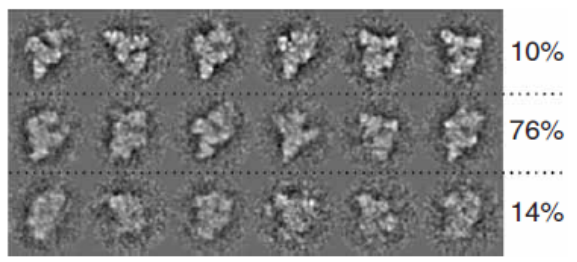

d

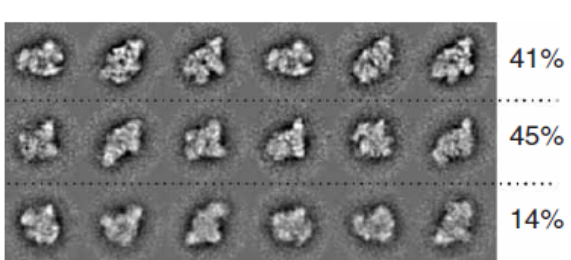

Figure 1.10: Effect of GraFix-based sample preparation on B complex spliceosomes and the $70 S$ ribosome. $(a, b)$ Uranyl formate-stained electron microscopic raw image of spliceosomes prepared by a conventional glycerol gradient (a) or GraFix (b). Scale bars: $40 \mathrm{~nm}$. Arrowheads show smaller broken parts and flexible elements. Insets show a similarly oriented spliceosomal class average. (c,d) Class averages obtained from a set of 5,000 raw images of non-GraFix-prepared (c) or GraFix-prepared (d) samples. Class averages were sorted vertically with respect to contrast and structural definition. GraFix treatment (d) generates computed class averages with improved contrast (top and middle; $86 \%$ of images), as compared to samples prepared by the conventional method (c), where only $10 \%$ of class averages (top) show relatively well defined structural features. Image courtesy of Kastner et al. [2008]. 


\section{Introduction}

by a buffer exchange step via dead volume gel filtration. Unfixated macromolecular complexes can be damaged in this step, but the chemically stabilized samples show no adverse effects with respect to buffer exchange. Because of these numerous advantages, the GraFix protocol has become a standard procedure in single particle electron microscopy. However, even treated by the GraFix protocol, particles still exhibit conformational heterogeneity - which has to be addressed by different methods.

\subsubsection{Macromolecular Crystallization additives}

Various compounds or small molecules can influence the crystallization behaviour of proteins. Among them are small molecules (often with multiple charged and polar groups), metal ions or substrate analogues which can directly interact with the protein, but also cosmostropes or solubilizing agents. Although their exact effects on crystal packing are not fully understood, these additives can significantly promote crystal formation [McPherson and Cudney, 2006, Larson et al., 2007]. Trifluoroethanol (TFE) has been known to stabilize the helical structure of proteins that were otherwise unstable or prone to misfolding [Shiraki et al., 1995]. While the introduction of heavy atoms usually serves to facilitate the solution of the phase problem with experimental phasing methods, such as isomorphous replacement, they may also facilitate crystallization [Lawson et al., 1991]. Large heavy metal clusters can affect the crystal packing by interposing between the protein particles or positioning in intramolecular cavities [Yonath et al., 1998]. Since crystallization requires a high degree of order and homogeneity, crystallization additives might pose promising reagents to lower heterogeneity in cryo-EM samples as well.

\subsection{Macromolecular complexes}

In this work, four macromolecular complexes were used: human and yeast spliceosomes from Saccharomyces cerevisiae, $70 \mathrm{~S}$ ribosomes from E. coli and bovine glutamate dehydrogenase. They are briefly introduced below. 


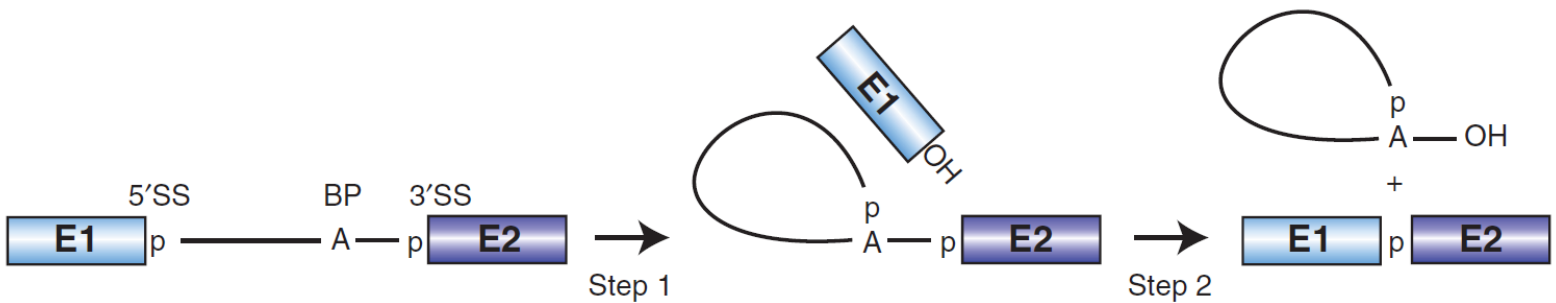

Figure 1.11: Schematic representation of the two-step mechanism of pre-mRNA splicing. Boxes and solid lines represent the exons (E1, E2) and the intron, respectively. The branch site adenosine is indicated by the letter A. The phosphate groups (p) at the $5^{\prime}$ and $3^{\prime}$ splice sites, which are conserved in the splicing products, are also shown. Image courtesy of Will and Lührmann [2011].

\subsubsection{The spliceosome}

Protein biosynthesis consists of transcription of genetic information into messenger RNA (mRNA) followed by translation of that very mRNA into proteins by the ribosome. In eukaryotes, the result of transcription is a precursor mRNA (pre-mRNA) containing coding regions (exons) and non-coding regions (introns).

During the splicing process, the introns are removed and the exons are ligated together in a two-step transesterification. This process is catalyzed and facilitated by the spliceosome.

The spliceosome is a large protein complex composed of five uridine-rich small nuclear ribonucleoproteins (snRNPs), named U1, U2, U4, U5 and U6 snRNP, and numerous non-snRNP-proteins. Each snRNP particle in turn consists of a uridine-rich short nuclear ribonucleic acid (snRNA) molecule and various snRNP-specific proteins. These components do not rigidly fit together but are highly dynamic. Furthermore, the spliceosome undergoes significant compositional and structural changes during the splicing cycle, gaining and losing snRNPs and proteins in the process as well as rearranging its RNA network [Staley and Guthrie, 1998]. These dynamic properties make the spliceosome a challenging target for three-dimensional structure determination because it exhibits a high degree of structural and conformational heterogeneity [Lührmann and Stark, 2009]. Current EM-maps of entire spliceosomes attain only low resolutions which are insufficient for identifying individual components. Labelling subunits and identifying their position via these labels is one way to gain a better understanding of the spatial arrangement of subunits within the spliceosome. And although better resolved structures of isolated snRNPs have been obtained, so far only the relatively small U1 snRNP could be completely mapped 


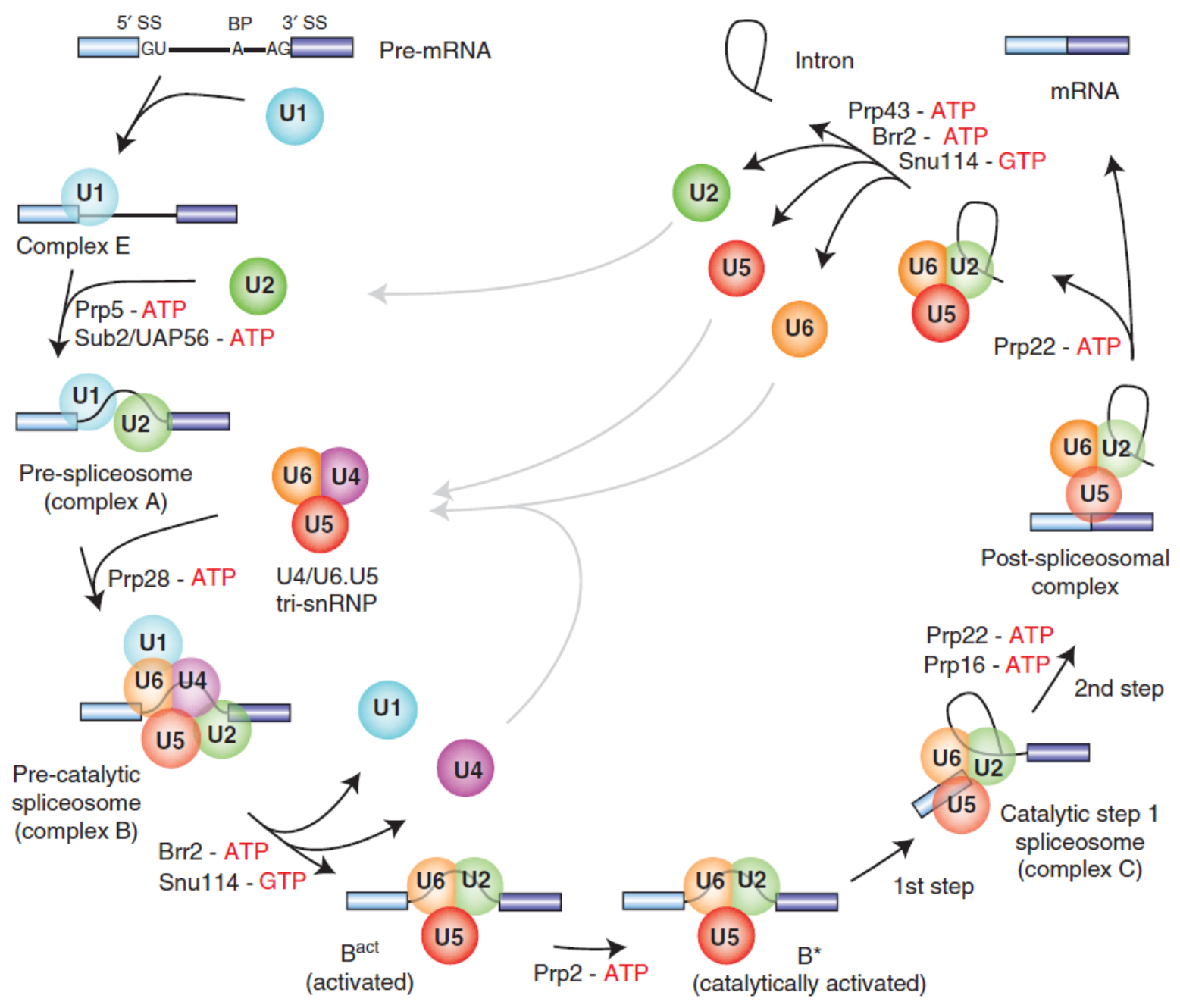

Figure 1.12: Canonical cross-intron assembly and disassembly pathway of the U2-dependent spliceosome. For simplicity, only the ordered interactions of the snRNPs (indicated by circles) but not those of non-snRNP proteins are shown. The various spliceosomal complexes are named according to the metazoan nomenclature. Exon and intron sequences are indicated by boxes and lines, respectively. The stages at which the evolutionarily conserved DExH/D-box RNA ATPases/helicases Prp5, Sub2/UAP56, Prp28, Brr2, Prp2, Prp16, Prp22 and Prp43, or the GTPase Snu114 act to facilitate conformational changes are indicated. Image courtesy of Will and Lührmann [2011]. 
with these techniques [Stark et al., 2001]. Recent crystal structures of the U1 snRNP have allowed this subunit to be elucidated in more detail [Pomeranz Krummel et al., 2009, Weber et al., 2010], but to put those subunit structures into perspective, EM-maps of entire spliceosomal complexes are helpful. One key towards obtaining higher resolution of 3D-EM reconstruction of the spliceosome is to better control its structural heterogeneity.

\section{The splicing cycle}

The spliceosome is assembled in situ onto the pre-mRNA in a stepwise process. In the first step, the $5^{\prime}$ end of the U1 snRNP binds to the 5' splice site of the pre-mRNA via base-pairing interactions. In a subsequent ATP-dependent step, the U2 snRNP associates with the branch site, resulting in the spliceosomal A complex (or pre-spliceosome). The binding of a pre-assembled U4/U6.U5 tri-snRNP to the A complex forms the B complex (or precatalytic spliceosome) which now contains all five snRNPs. However, it is still catalytically inactive and needs to undergo structural rearrangements, including the release of the U1 and U4 snRNPs, resulting in the $\mathrm{B}^{a c t}$ complex (or activated spliceosome). The $\mathrm{B}^{\text {act }}$ complex is then catalytically activated and undergoes further remodelling in a Prp2 and ATP mediated step to yield the B* complex (the catalytically activated spliceosome). The $\mathrm{B}^{*}$ complex catalyzes the first step of splicing, which creates the C complex. Further rearrangements occur before the second splicing step, in which the 5'-and $3^{\prime}$ exons are ligated. After that, the mature mRNA is released and the post-spliceosomal complex disassociates into the U2, U5 and U6 snRNPs, which are reused for further splicing cycles, and the intron-lariat, which is decomposed.

\subsubsection{The ribosome}

The ribosome is a large ribonucleoprotein complex which synthesizes proteins in the cell by translating the information from the messenger RNA into a sequence of amino acids and stringing them together. It consists of two subunits, which can rotate relative to each other in a ratchet-like manner during protein synthesis [Horan and Noller, 2007]. The eukaryotic ribosome, with a sedimentation coefficient of 80 Svedberg units (80S), is about 50\% larger than the prokaryotic ribosome (70S) and both differ significantly in protein composition, with only 


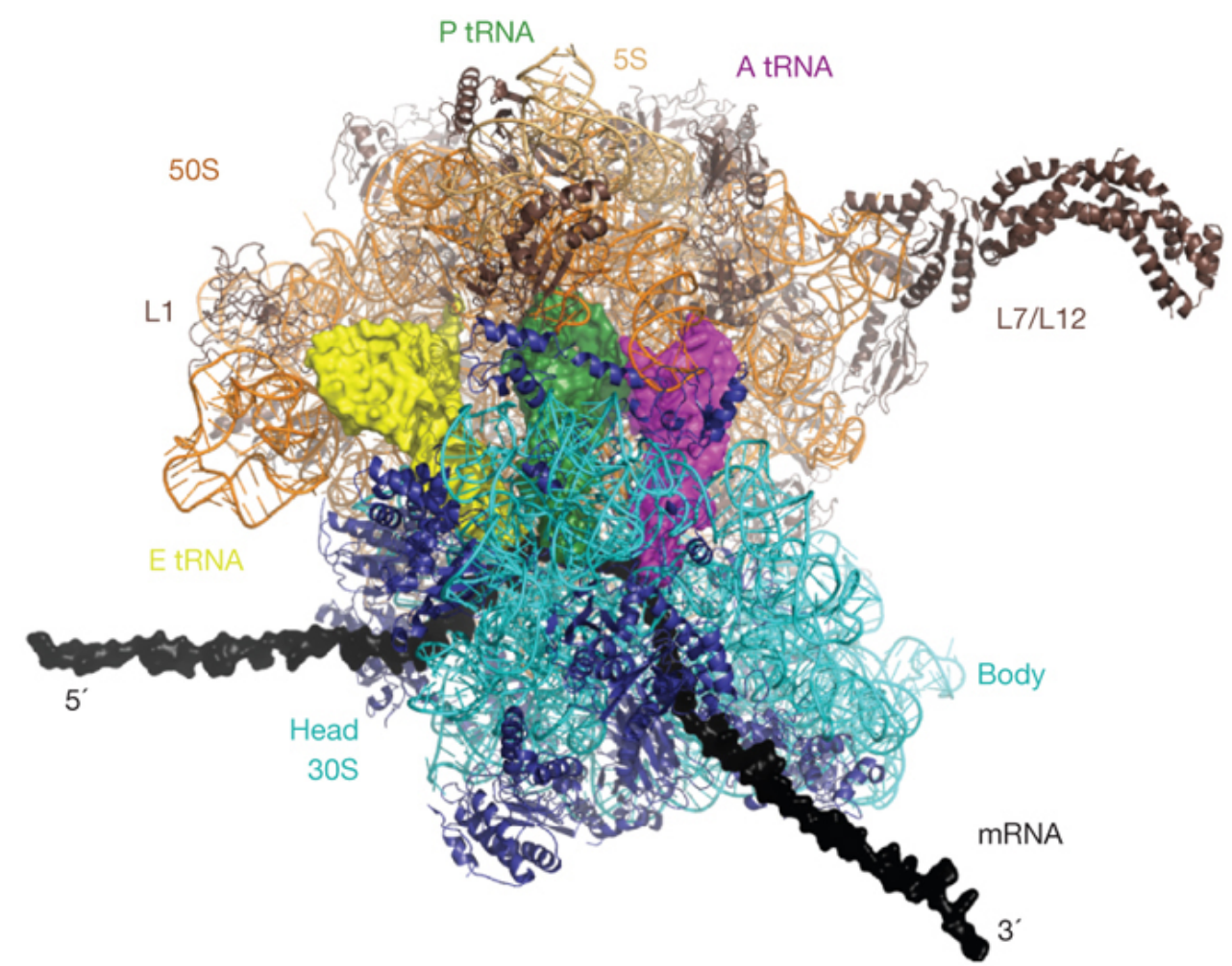

Figure 1.13: Top view of the 70S ribosome with mRNA and A- P- and E-site tRNAs. Image courtesy of Schmeing and Ramakrishnan [2009].

one third of the eukaryotic ribosomal proteins possessing a counterpart in bacteria. Despite these differences, they are quite similar in core shape and function [Schmeing and Ramakrishnan, 2009, Ben-Shem et al., 2010]. The ribosome consists in large parts of RNA strands glued into shape by proteins. The large subunit ( $60 \mathrm{~S}$ in eukaryotes, $50 \mathrm{~S}$ in bacteria) contains the active site. It handles the amino acids brought in by transfer-RNA (tRNA) and creates the peptide bonds between them. The smaller subunit (40S in eukaryotes, $30 \mathrm{~S}$ in bacteria) decodes the information from the mRNA and pairs each codon with the anticodon in the proper tRNA. Besides the intersubunit movement, the head domain of the small subunit has the ability to rotate, which plays a role in controlling the position of the tRNAs in the ribosome [Zhang et al., 2009].

\subsubsection{Glutamate dehydrogenase}

Glutamate dehydrogenase (GDH) is an enzyme present in eukaryotic mitochondria where it plays a crucial role in nitrogen and carbon metabolism. It reversibly catalyzes the oxidative de-amination of L-glutamate to 2-oxoglutarate ( $\alpha$-ketoglutarate) using $\mathrm{NADP}(\mathrm{H})$ or $\mathrm{NAD}(\mathrm{H})$. 


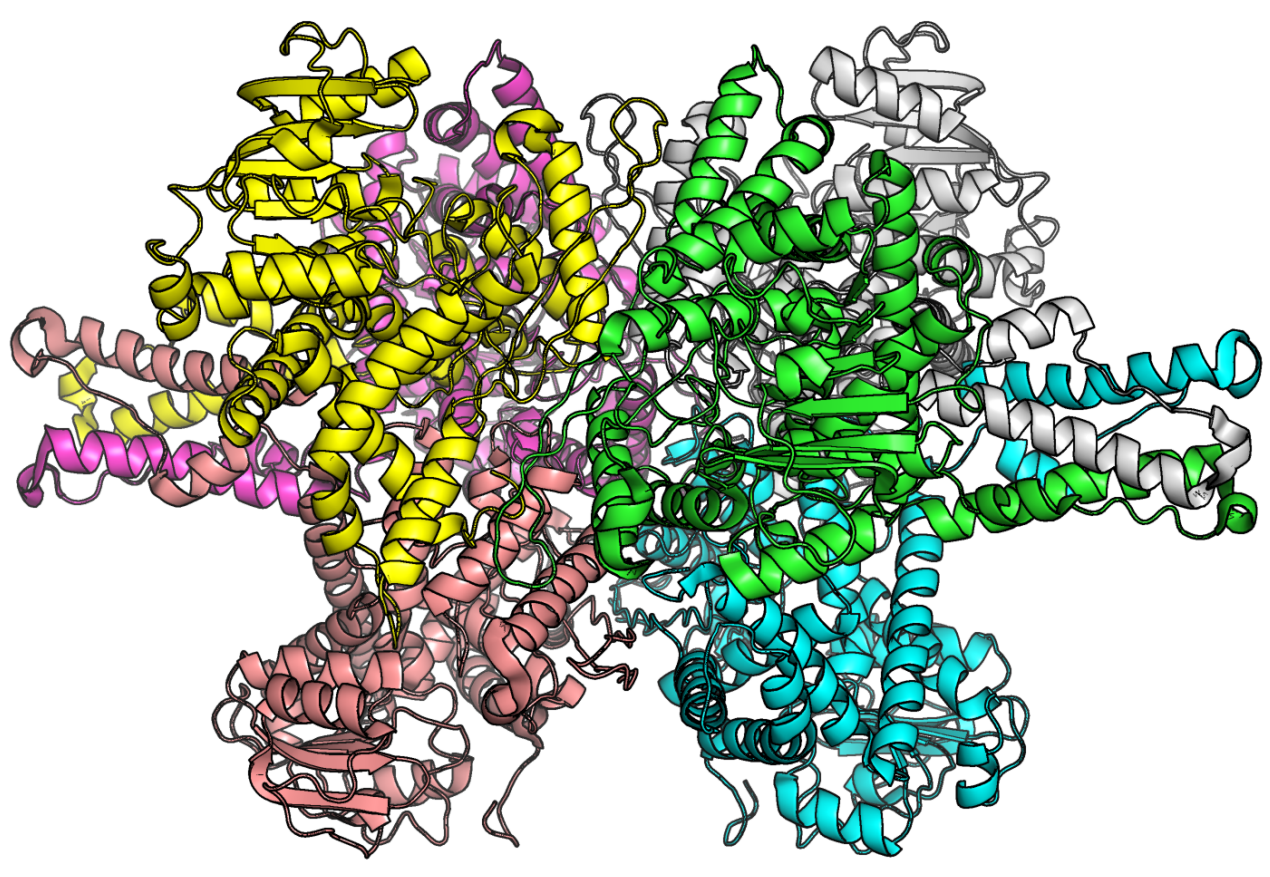

Figure 1.14: Crystal structure of bovine glutamate dehydrogenase with the individual subunits coloured differently. Image generated from PDB entry 3MW9 [Peterson and Smith, 1999].

Its structure consists of six identical associated subunits, arranged in a dimer of two trimers stacked on top of each other. The two subunits are separated by a cleft, giving the protein a 'clam-like' appearance [Peterson and Smith, 1999].

\subsection{Aim of the work}

While sample heterogeneity may offer methods to study conformational dynamics or enable time-resolved analysis of biochemical processes, it remains a challenge that has to be overcome in order to further improve resolution in single-particle EM. The methods to cope with heterogeneity in silico include the separation of unwanted conformations from the dataset, thus limiting the data available for reconstruction. This work approaches the problem from the side of sample preparation. Limiting heterogeneity before image aquisition, for example by enriching the population of a stable conformation, would tremendously benefit the image processing steps. If fewer images had to be sorted out, the recorded data could be used more efficiently and the quality of the reconstruction would be improved. 
First, the structural diversity of macromolecular complexes is emphasized by examining the rearrangement of the spliceosomal $\mathrm{B}^{a c t}$-complex during catalytic activation. Subsequently, two methods are explored to modify the GraFix protocol in order to obtain more homogeneous samples. Chemical modification attempts to impose order in a spliceosome sample by admixing chemical additives to the GraFix gradient and studying their effect on the conformational homogeneity of the sample. The effect is quantified by means of Fourier-ring-correlation analysis. Finally, cryo-fixation is introduced, which applies the GraFix protocol at temperatures below $0{ }^{\circ} \mathrm{C}$ in order to stabilize a thermodynamically favoured conformation. To establish the "CryoFix protocol", preliminary tests are run with glutamate dehydrogenase, before it is applied to ribosome samples. The intersubunit rotation of ribosomes at various fixation and preparation temperatures are analyzed to determine the effect of the new preparation protocol. 


\section{Materials \& Methods}

\subsection{Materials}

\subsubsection{Chemicals}

\begin{tabular}{ll}
\hline Chemical & Supplier \\
\hline 4-(2-hydroxyethyl)-1-piperazineethanesulfonic acid (HEPES) & Sigma-Aldrich \\
Acetic acid ester, ultrapure grade & Fluka-Riedel-de-Haen \\
Acetobutyrate cellulose in ethyl acetate $0.5 \%$ (Triafol) & Sigma-Aldrich \\
Acetone & Merck \\
Adenosinetriphosphate (ATP) & Amersham Biosciences \\
Alkylbenzyldimethylammoniumchlorid $0.5 \%$ (Osvan solution) & Sigma-Aldrich \\
Ammoniumperoxodisulfate (APS) & Merck \\
Cadmium chloride, CdCl 2 & Fluka-Riedel-de-Haen \\
Dithiothreitol (DTT) & Carl Roth \\
Ethanol & Merck \\
Ethylenediaminetetraacetic acid (EDTA) & Sigma-Aldrich \\
Glutaraldehyde & Electron Microscopy Sciences \\
Glycerol & Merck \\
Glycine & Merck \\
Hexatantalum tetradecabromide, [Ta $\left.{ }_{6} \mathrm{Br}_{12}\right]^{2+} \times \mathrm{x}_{2} \mathrm{Br}^{-}$ & Jena Bioscience \\
Hydrofluoric acid & Fluka-Riedel-de-Haen \\
Magnesium chloride & Merck \\
Millipore water & Millipore GmbH \\
Polyethyleneglycol (PEG) 4000 & Fluka-Riedel-de-Haen \\
\hline
\end{tabular}




\begin{tabular}{ll}
\hline Chemical & Supplier \\
\hline Polyethyleneglycol (PEG) 8000 & Merck, Darmstadt \\
Potassium chloride & Merck \\
Potassium hexachloroplatinate, $\mathrm{K}_{2}\left[\mathrm{PtCl}_{6}\right]$ & Hampton Research \\
Potassium hydroxide & Sigma-Aldrich \\
Rotiphorese Gel 40 & Carl Roth \\
Sodium chloride & Merck \\
Sodium sulfate, $\mathrm{Na}_{2} \mathrm{SO}_{4}$ & Sigma-Aldrich \\
Spermidine & Fluka-Riedel-de-Haen \\
Spermine & Fluka-Riedel-de-Haen \\
Sucrose & Merck \\
SYPRO Ruby stain & Invitrogen \\
Tetramethylethylenediamine (TEMED) & Sigma-Aldrich \\
Triflouroethanol, $\mathrm{CF}_{3} \mathrm{CH}_{2} \mathrm{OH}$ & Carl Roth \\
Tris(hydroxymethyl)aminomethane (TRIS) & Sigma-Aldrich \\
Uranyl formate & Polyscience Inc. \\
\hline
\end{tabular}

Unless otherwise noted, water was always used Milipore-filtered. 


\subsubsection{Buffers}

All buffers were prepared using Millipore-filtered water.

\begin{tabular}{|c|c|}
\hline Buffer & Composition \\
\hline \multirow[t]{3}{*}{ Gradient buffer for human B-complex preparation } & 20 mM Hepes-KOH pH 7.9 \\
\hline & $150 \mathrm{mM} \mathrm{KCl}$ \\
\hline & $20 \mathrm{mM} \mathrm{MgCl}_{2}$ \\
\hline \multirow[t]{2}{*}{ Gradient buffer for glutamate dehydrogenase } & 20 mM Hepes-KOH pH 7.9 \\
\hline & $150 \mathrm{mM} \mathrm{KCl}$ \\
\hline \multirow[t]{2}{*}{ Quenching solution for glutamate dehydrogenase } & 2 M glycin \\
\hline & $200 \mathrm{mM}$ Tris $\mathrm{pH} 7.4$ \\
\hline \multirow[t]{3}{*}{ HKM-7 } & 20 mM Hepes-KOH pH 7.5 \\
\hline & $100 \mathrm{mM} \mathrm{KCl}$ \\
\hline & $7 \mathrm{mM} \mathrm{MgCl}_{2}$ \\
\hline \multirow[t]{5}{*}{ HKM-7PA } & 20 mM Hepes-KOH pH 7.5 \\
\hline & $100 \mathrm{mM} \mathrm{KCl}$ \\
\hline & $7 \mathrm{mM} \mathrm{MgCl}$ \\
\hline & 20.6 mM Spermine \\
\hline & 0.4 mM Spermidine \\
\hline \multirow[t]{4}{*}{ HAKM-7 } & 20 mM Hepes-KOH pH 7.5 \\
\hline & $70 \mathrm{mM} \mathrm{NH}_{4} \mathrm{Cl}$ \\
\hline & $30 \mathrm{mM} \mathrm{KCl}$ \\
\hline & $7 \mathrm{mM} \mathrm{MgCl} 2$ \\
\hline \multirow[t]{6}{*}{ HAKM-7PA } & 20 mM Hepes-KOH pH 7.5 \\
\hline & $70 \mathrm{mM} \mathrm{NH}_{4} \mathrm{Cl}$ \\
\hline & $30 \mathrm{mM} \mathrm{KCl}$ \\
\hline & $7 \mathrm{mM} \mathrm{MgCl}_{2}$ \\
\hline & $0.6 \mathrm{mM}$ Spermine \\
\hline & 0.4 mM Spermidine \\
\hline
\end{tabular}




\subsubsection{Laboratory materials}

\begin{tabular}{|c|c|}
\hline Item & Supplier \\
\hline Amicon Centrifugal Filter Units & Milipore GmbH \\
\hline Carbon rods, highest grade & Ringsdorff Werke GmbH \\
\hline Centrifuge Tubes & Beckmann \\
\hline Copper EM grids, 3.05 mm, 300 mesh, square & Plano \\
\hline Quantifoil grids & Quantifoil Micro Tools \\
\hline Ethane (liquid) & Messer \\
\hline Filter paper $90 \mathrm{~mm}$, No. 4 and 5 & Whatman \\
\hline Glass slides (76 x 26 mm) & Gerhard Menzel GmbH + Co. KG \\
\hline Mica, $75 \times 25 \mathrm{~mm}$ & Plano \\
\hline Nitrogen (liquid) & Messer \\
\hline PD MidiTrap G25 columns & GE Healthcare \\
\hline \multirow[t]{2}{*}{ Teflon preparation block (volume: $25 \mu \mathrm{l}-200 \mu \mathrm{l}$ ) } & Manufactured by the precision \\
\hline & engineering team, MPI-BPC \\
\hline
\end{tabular}

\subsubsection{Special Equipment}

\begin{tabular}{ll}
\hline Item & Source \\
\hline Cryo electron microscope CM200 FEG & Philips \\
Cryo electron microscope Titan Krios & FEI \\
Edwards E12E vacuum coating unit & BOC Edwards \\
Fraction Collector FRAC-200 & Amersham Pharmacia Biotech \\
FLA-7000 bio-imaging system & Fujifilm \\
Gradient Master & BioComp Instruments, Inc. \\
Heraeus Desk Centrifuge Biofuge Fresco & Heraeus Sepatech GmbH \\
Nanodrop ND-1000 Spectrophotometer & Peqlab Biotechnologie \\
Peristaltic Pump LKB-Pump P-1 & Amersham Pharmacia Biotech \\
Room temperature EM specimen holder & Philips, \\
Sorvall Micro-Ultracentrifuge Discovery M150 & Thermo Scientific
\end{tabular}




\begin{tabular}{ll}
\hline Item & Source \\
\hline Sorvall Rotor S55S & Thermo Scientific \\
Sorvall Rotor Surespin 630 & Thermo Scientific \\
Sorvall Rotor TH660 & Thermo Scientific \\
Sorvall Ultracentrifuge Discovery 90SE & Thermo Scientific \\
Sorvall Ultracentrifuge WX Ultra 90 & Thermo Scientific \\
TemCam F415 (slow scan 4K x 4K CCD camera) & Tietz Video Systems \\
Vacuum glow discharge apparatus & Manufactured by the precision \\
& engineering team, MPI-BPC \\
Vitrobot Mark IV & FEI \\
\hline
\end{tabular}

\subsubsection{Software}

\begin{tabular}{ll}
\hline Program & Source \\
\hline ActivePerl & ActiveState, Vancouver \\
BOXER from EMAN Suite & Steven Ludtke, NCMI, Houston \\
ClassTest & Mario Lüttich, Stark lab \\
Compass Run Simulation Software & Thermo Scientific \\
CowHide & Mario Lüttich, Stark lab \\
IMAGIC-5 & Image Science, Berlin \\
John Henry & Boris Busche, Stark lab \\
Python & Python Software Foundation, Delaware \\
TVIPS EM-MENU & Tietz Video System, Gauting \\
\hline
\end{tabular}




\subsection{Biochemical Methods}

\subsubsection{Assembly and purification of the human spliceosomal B-complex}

Samples of the human spliceosomal B-complex were assembled in collaboration with Elmar Wolf. In a pre-incubation step, the $\left[{ }^{32} \mathrm{P}\right]$ labeled MS2-tagged pre-mRNA was treated with a 20-fold excess of MS2 coat protein fused to maltose binding protein (MS2-MBP) for 30 minutes at $4{ }^{\circ} \mathrm{C}$. The splicing reaction contained $40 \%$ [v/v] HeLa cell nuclear extract, $20 \mathrm{mM} \mathrm{KCl}, 3$

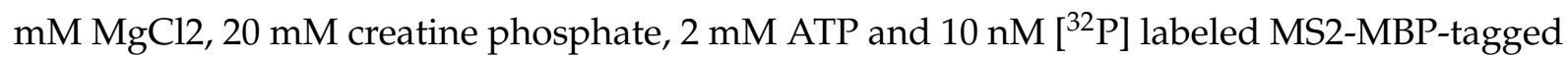
pre-mRNA. Spliceosomal complexes were allowed to form at $30^{\circ} \mathrm{C}$ in a standard heating block. After 7 minutes, the splicing reaction was stopped by chilling the samples on ice. The splicing reactions were loaded onto $10-30 \%$ [v/v] glycerol gradients in $36 \mathrm{~mL}$ cups and centrifuged for $16 \mathrm{~h}$ at $25,000 \mathrm{rpm}$ and $4{ }^{\circ} \mathrm{C}$ in a Surespin 630 rotor. The gradients were harvested manually in $1,500 \mu \mathrm{L}$ fractions. The peak fractions were identified in a scintillation counter, pooled and loaded onto a buffer-equilibrated column containing $400 \mu \mathrm{L}$ amylose beads. The matrix was washed with 4 column volumes of buffer. The complexes were eluted dropwise with $1 \mathrm{~mL}$ of elution buffer (buffer containing $15 \mathrm{mM}$ maltose) and subsequently loaded on a GraFix gradient.

\subsubsection{Polyacrylamide gel electrophoresis}

Native polyacrylamide gel electrophoresis (PAGE) was used to analyze the degree of glutaraldehyde fixation in the glutamate dehydrogenase samples. Gels contained Tris buffer $(0.375 \mathrm{M}), 10 \%$ Rotiphorese Gel40 and 18.75\% sucrose. Polymerization was initiated by addition of $3.3 \mu \mathrm{L} / \mathrm{mL}$ 10\% APS solution and $3.3 \mu \mathrm{L} / \mathrm{mL}$ TEMED. Samples were concentrated in Amicon centrifugal filter units before loading. Gels were stained with Sypro-Ruby and analyzed via fluorescence scanning.

\subsection{GraFix sample-preparation}

The GraFix protocol [Kastner et al., 2008] was used on all samples. The GraFix protocol combines an ultracentrifugation step with an increasing gradient of glutaraldehyde as a crosslinking reagent. The gradients were prepared by underlayering a phase with low density buffer 
(glycerol or sucrose) with a phase of high density buffer that also contained the glutaraldehyde. The gradients are formed in a gradient former (Gradient Master, BioComp Instruments, Canada) with preprogrammed parameters. After forming, the gradients were stored at $4{ }^{\circ} \mathrm{C}$ for one hour. Before loading, a cushion of low density buffer without glutaraldehyde can be layered onto the gradient to eliminate possible interaction between glutaraldehyde and the sample buffer. The samples in this work were loaded without cushion. The glutamate dehydrogenase gradients were run in $2.2 \mathrm{~mL}$ cups in a Sorvall Discovery M150 micro-ultracentrifuge in a Sorvall S55S rotor. Spliceosome fixations were performed in $4.4 \mathrm{~mL}$ cups in a Sorvall Discovery $90 \mathrm{SE}$ ultracentrifuge with a Sorvall TH660 rotor. Ribosome fixations were performed in $4.4 \mathrm{~mL}$ cups in a Sorvall WX Ultra 90 ultracentrifuge with a Sorvall TH660 rotor. After centrifugation, the gradients were fractionated from the bottom with an automatic fraction collector or by hand from the top (glutamate dehydrogenase) and stored at $4{ }^{\circ} \mathrm{C}$.

\subsubsection{Spliceosomal remodeling}

Purified samples that had already been fixated with $0.1 \%$ glutaraldehyde in a $10 \%-30 \%$ [v/v] glycerol gradient were kindly provided by Zbigniew Warkocki, department of cellular biochemistry, MPI-BPC Göttingen.

\subsubsection{Chemical modification}

For the chemical modification of the GraFix-protocol, additives as listed below were added to the high density buffer of GraFix gradients at a final glutaraldehyde concentration of $0.1 \%$. The gradients were centrifuged for $1 \mathrm{~h} 47 \mathrm{~m}$ at $60,000 \mathrm{rpm}$ and $4{ }^{\circ} \mathrm{C}$. Fractions were collected automatically (each $\sim 185 \mu \mathrm{L}$ ). The peak fractions were determined by scintillation count measurement of the samples. An unmodified sample was also prepared as control. 


\begin{tabular}{cc}
\hline Sample modified by & Concentration in high density gradient fraction \\
\hline \hline Tantalum bromide, $\mathrm{Ta}_{6} \mathrm{Br}_{14}$ & $0.5 \mathrm{mM}$ \\
Potassium hexachloroplatinate, $\mathrm{K}_{2}\left[\mathrm{PtCl}_{6}\right]$ & $2.8 \mathrm{mM}$ \\
Sodium sulfate, $\mathrm{Na}_{2} \mathrm{SO}_{4}$ & $20 \mathrm{mM}$ \\
Triflouroethanol, $\mathrm{CF}_{3} \mathrm{CH}_{2} \mathrm{OH}$ & $30 \%[\mathrm{v} / \mathrm{v}]$ \\
Cadmium chloride, $\mathrm{CdCl}_{2}$ & $12 \mathrm{mM}$ \\
Polyethyleneglycol 4000 & $50 \mathrm{mM}(20 \%[\mathrm{v} / \mathrm{v}])$ \\
Polyethyleneglycol 8000 & $25 \mathrm{mM}(20 \%[\mathrm{v} / \mathrm{v}])$ \\
\hline
\end{tabular}

\subsubsection{Cryo-Preparation}

The experiments to establish the protocol to fixate samples at $-9.9{ }^{\circ} \mathrm{C}$ were conducted in collaboration with Ulrich Steuerwald, department of cellular biochemistry, MPI-BPC, who also kindly provided purified samples of bovine glutamate dehydrogenase.

\subsubsection{Glutamate dehydrogenase}

For each run, four gradients (glycerol 5-20\% [v/v], no cushion) with different maximum concentrations of glutaraldehyde were prepared: $0.01 \%, 0.025 \%, 0.05 \%$ and $0.1 \%$. Each gradient was loaded with $19.5 \mu \mathrm{g}$ of glutamate dehydrogenase in $130 \mu \mathrm{L}$ protein dilution buffer. The $+4{ }^{\circ} \mathrm{C}$ gradients were run for $18 \mathrm{~h}$ at $22,000 \mathrm{rpm}$, the $-9.9^{\circ} \mathrm{C}$ gradients were run for $16 \mathrm{~h}$ at 33,000 $\mathrm{rpm}$. The gradients were manually harvested into $200 \mu \mathrm{L}$ fractions and each fraction was treated with $50 \mu \mathrm{L}$ quenching solution. For storage, $200 \mu \mathrm{L}$ glycerol was added to each fraction and the samples were stored at $-20{ }^{\circ} \mathrm{C}$. Another cryo-run was set up with $0.1 \%, 0.3 \%, 0.8 \%$ and $1.5 \%$ glutaraldehyde as well as an unfixated sample. The gradients were run for $18 \mathrm{~h}$ at $35.000 \mathrm{rpm}$ and then treated as above. The sample containing fractions were loaded on a $16 \%$ SDS-PAGE gel and a 4\% native PAGE gel respectively and developed with SYPRO Ruby stain.

\subsubsection{S ribosome}

The experiments to compare the effects of cryo-fixation with the standard GraFix protocol were conducted in collaboration with Niels Fischer. Purified samples of 705 ribosomes from 
Escherichia coli were kindly provided by Frank Peske, department of physical biochemistry, MPI-BPC Göttingen.

\section{Standard fixation}

For the $+4{ }^{\circ} \mathrm{C}$ GraFix preparation three gradients (HKM-7, sucrose $10-40 \%$ [w/v], no cushion) with a maximum glutaraldehyde concentration of $0.15 \%$ and one gradient without glutaraldehyde were prepared. Each gradient was loaded with 70 pmol sample in $20 \mu \mathrm{L}$ HKM-7PA buffer. The gradients were run for $18 \mathrm{~h}$ at 20,000 rpm. The gradients were fractionated from bottom to top and treated with $15 \mu \mathrm{L} 2 \mathrm{M}$ glycine in HKM-7. Nanodrop optical density (OD)-measurement of the glutaraldehyde-free gradient confirmed the sample peak in fractions 10 and 11 of 23.

\section{Cryo-fixation}

For the $-9.9^{\circ} \mathrm{C}$ GraFix preparation three gradients (HKM-7, sucrose $10-40 \%$ [w/v], no cushion) with a maximum glutaraldehyde concentration of $1 \%$ and one gradient without glutaraldehyde were prepared. Each gradient was loaded with 70 pmol sample in $20 \mu \mathrm{L}$ HKM-7PA buffer. The gradients were run for $20 \mathrm{~h}$ at $22,000 \mathrm{rpm}$. The gradients were fractionated from bottom to top and treated with $100 \mu \mathrm{L} 2 \mathrm{M}$ glycine in HKM-7. Nanodrop OD-measurement of the glutaraldehyde-free gradient confirmed the sample peak in fractions 13 and 14 of 24.

\subsection{Sample preparation for single-particle EM}

\subsubsection{Preparation of carbon film}

Mica was freshly split and placed into an Edwards E12E vacuum coating unit in which carbon rod electrodes sputter carbon under high voltage. A custom made metal basket, where the carbon was reflected onto the mica by specifically placed object slides, was used to prevent direct carbon aggregation and achieve indirect coating.

\subsubsection{Preparation of EM-grids coated with holey carbon film}

A light microscopy object slide was cleaned with ethanol and immersed for 15 minutes in Osvan solution (alkylbenzyldimethylammonium chloride $0.5 \%$ ) to render the glass surface 


\section{Materials \& Methods}

hydrophobic. The slide was rinsed in water and left to dry. An atmosphere of high humidity was generated in a repurposed glove box with boiling water. In this atmosphere, the slide was incubated on a cooled aluminium block so that water condensed on the glass in small droplets. The slide was lifted on one side and $1 \mathrm{~mL}$ of $0.5 \%$ Triafol (acetobutyrate cellulose) in ethyl acetate was pipetted over it. After evaporation of the ethyl acetate, the Triafol formed a thin film with small holes where the water droplets had been. This film was detached from the slide by treating it with $0.02 \%$ hydrofluoric acid for $30 \mathrm{~s}$ and was then floated on water. Copper grids were cleaned in ethanol in an ultrasonic water bath and then placed on the film with the shiny side up. Once covered with copper grids, the Triafol film was transferred onto a new object slide covered with a paper sticker so that the copper grids were between the Triafol film and the paper. After air-drying, the slide was then sputtered with carbon as described above, albeit this time directly. Finally, the Triafol was removed by placing the grids on a filter paper soaked with ethyl acetate in a closed glass jar overnight. The grids were inspected under a light microscope and sorted for hole-size and quality.

\subsubsection{Preparation of negative stain samples}

A $2.5 \%$ uranyl formate solution was prepared by dissolving $25 \mathrm{mg}$ of uranyl formate in 1,000 $\mu \mathrm{L}$ water, adding $25 \mu \mathrm{L} 1 \mathrm{M} \mathrm{NaOH}$ and vortexing vigorously for several minutes. The solution was centrifuged at $13,000 \mathrm{rpm}$ and $4{ }^{\circ} \mathrm{C}$ for 10 minutes to sediment possibly remaining uranyl formate crystals and impurities. The stain is light sensitive and had to be used freshly. The sample was filled into a well in a teflon preparation block that holds $25 \mu \mathrm{L}$. A small piece of carbon coated mica (approximately $3 \times 4 \mathrm{~mm}$ ) was cut and inserted into the well in a $45^{\circ}$ angle so that the carbon film partially detached and floated on the surface. After a variable adsorption time - mainly dependent on sample concentration from 5 minutes to several hours - the mica was taken out of the well and excess buffer was removed by blotting with a filter paper from the side. The mica was then inserted into a larger well $(120 \mu \mathrm{L})$ containing uranylformate solution and the carbon film was floated completely. After 2 minutes the carbon film was taken up with a holey carbon coated copper grid, excess stain was blotted and the grid was dried under a light bulb. 


\begin{tabular}{cc}
\hline Sample & Adsorption time \\
\hline \hline Human spliceosomal B-complex & 2 hours \\
Yeast B ${ }^{\text {act }}$ and B & 16 hours \\
\hline
\end{tabular}

\subsubsection{Preparation of unstained cryo-samples}

Sucrose was removed from the sample with a buffer exchange procedure via dead-volume gel filtration using PD MidiTrap G-25 columns. After equilibrating the columns with 3 column volumes of HAKM-7PA, $1 \mathrm{~mL}$ of sample was loaded and run into the column. Subsequently, 1.6 $\mathrm{mL}$ of buffer were loaded and five drop fractions of the eluate were collected. Fraction number 4 was used for the preparation of the unstained cryo-samples. $5 \mu \mathrm{L}$ of the sample were pipetted onto a carbon-coated and glow-discharged quantifoil grid covered with thin carbon foil and incubated in the Vitrobot at a relative humidity of $100 \%$. The grids were plunged into liquid ethane to vitrify the sample and then transferred to and stored in liquid nitrogen.

\begin{tabular}{lccccc}
\hline Sample name & Centrifuge temp. & Vitrobot temp. & Incubation time & Blot time & Blotforce \\
\hline \hline P17 & No GraFix & $4{ }^{\circ} \mathrm{C}$ & $45 \mathrm{~s}$ & 10 & 12 \\
P10 & No GraFix & $20^{\circ} \mathrm{C}$ & $30 \mathrm{~s}$ & 8 & 12 \\
P12 & $4{ }^{\circ} \mathrm{C}$ & $4{ }^{\circ} \mathrm{C}$ & $30 \mathrm{~s}$ & 8 & 12 \\
P11 & $4{ }^{\circ} \mathrm{C}$ & $20^{\circ} \mathrm{C}$ & $30 \mathrm{~s}$ & 8 & 12 \\
P15 & $-9.9{ }^{\circ} \mathrm{C}$ & $4{ }^{\circ} \mathrm{C}$ & $50 \mathrm{~s}$ & 10 & 10 \\
P16 & $-9.9^{\circ} \mathrm{C}$ & $20^{\circ} \mathrm{C}$ & $50 \mathrm{~s}$ & 10 & 10 \\
\hline
\end{tabular}

\subsection{Electron microscopy analysis}

\subsubsection{Transmission electron microscopy}

TEM images of the spliceosomal complexes were recorded with a Philips CM200 FEG electron microscope at an acceleration voltage of $160 \mathrm{kV}$. Images were recorded on a $4 \mathrm{~K} \times 4 \mathrm{~K}$ Charge Coupled Device (CCD) camera with 2-fold binning at a nominal magnification of 115k-fold. The images were recorded in series of $5 \times 5$ spotscans with a $20 \%$ overlap. TEM images of the 


\section{Materials \& Methods}

ribosomes were recorded with an FEI Titan Krios electron microscope on a 4K x 4K eagle (p10) or falcon detector (all others) with 2-fold binning at a nominal 59k-fold magnification. The images were recorded in stacks of $3 \times 3$ spotscans with non-overlapping images.

\subsubsection{Raw image processing}

Each spotscan of the spliceosomal samples was combined into a large micrograph using the TVIPS EM-MENU software. Single particles were selected from the micrographs using the semi-automated Boxer program from the EMAN software package [Ludtke et al., 1999]. The spot series images of the ribosomal samples were not combined, but directly subjected to a semiautomated picking process with the John Henry software [in-house software, programmed by Boris Busche]. Selected particles were extracted from the micrograph as individual single images and appended into a single file. Cryo-images were subjected to CTF- and astigmatism correction using multivariate statistical analysis, followed by classification of the individual power spectra [Sander et al., 2003a]. The images were pixel-binned, a soft circular mask was applied and the images were bandpass filtered to exclude very high and very low spatial frequencies. Extraction and preparation of the images was performed with the IMAGIC-5 software [van Heel et al., 1996].

\subsubsection{Image Processing}

Image processing of the extracted images was performed with the IMAGIC-5 software [van Heel et al., 1996] and the CowCluster program from the CowHide software package [in-house software, programmed by Mario Lüttich]. Spliceosomal images were subjected to a referencefree alignment on the 2D-level until stable class averages were obtained. These were directly analyzed in the case of the remodeling studies. For the chemical modification studies, the heterogeneity of the dataset was analyzed by Fourier-ring-correlation [van Heel, 1987b]. Ribosome images were aligned to references projected from a library with multiple rotational states of the subunits [Fischer et al., 2010]. Supervised classification was used to analyze the distribution of conformational states with respect to subunit rotation, from which sample homogeneity could be determined. This allowed the effects of glutaraldehyde stabilization at different temperatures to be examined. 


\section{Results}

\subsection{Spliceosomal remodeling EM-studies}

Warkocki et al. [2009] have investigated the catalytic steps of splicing in an in vitro splicing complementation system. One conclusion from the biochemical data was that the protein Prp2 facilitates catalytic activation by remodeling the spliceosome. To verify and visualize this structural change, the yeast spliceosomal B-complex has been examined by negative stain electron microscopy prior to and after catalytic activation.
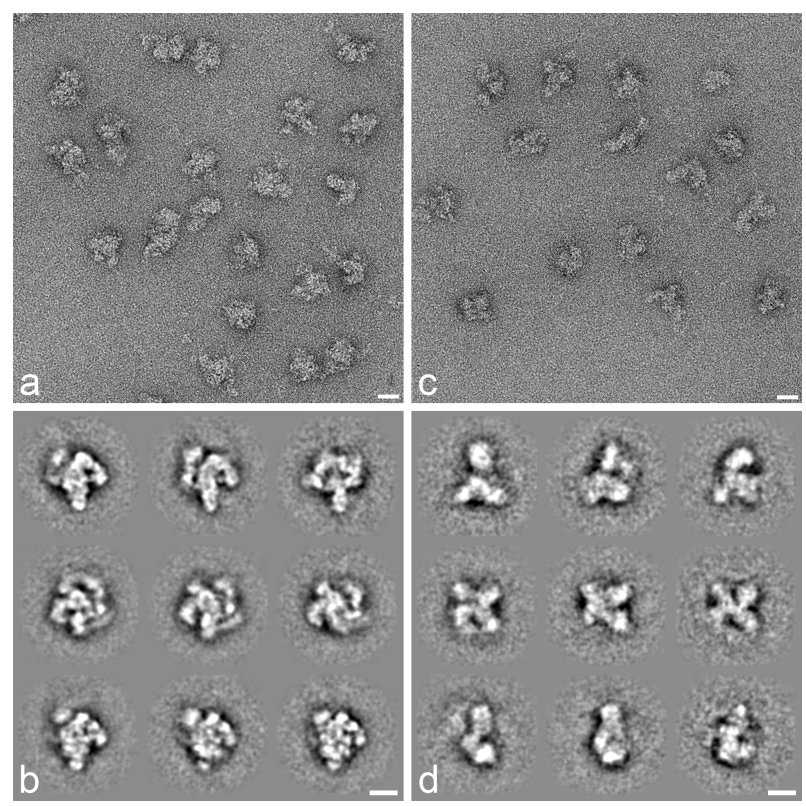

Figure 3.1: EM images of yeast spliceosomes before and after catalytic activation by Prp2. $(a, c)$ Typical raw images of spliceosomes before (a) and after (c) catalytic activation. (b,d) Typical class averages of spliceosomes before (b) and after (d) catalytic activation by Prp2. In $b$ and $d$ the three major views found in the respective datasets are shown. The white scale bar refers to $20 \mathrm{~nm}$. 


\section{Results}

Two datasets have been recorded: $\mathrm{B}^{\text {act }}$ (before activation) with 7,057 images and $\mathrm{B}^{*}$ (after activation) with 6,780 images respectively. Both datasets were subjected to independent reference-free alignment and classification [Penczek et al., 1992] over several rounds until a stable selection of class-averages was obtained. The datasets showed noticeably different class averages, supporting the assumption of a conformational change. Each dataset also had a number of dominant views - characteristic class averages that appeared in notable quantity.

A set of references, consisting of the three dominant views of each respective dataset, was generated and both datasets were aligned separately to the combined references. The resulting class averages were analyzed numerically and it was examined how many images aligned to each particular reference. Unsurprisingly, both datasets contained images that aligned to references from the respective other dataset (termed "foreign" in the following). However, the number of particles that aligned to one of their respective dataset's own references was significantly higher. Furthermore, the class averages obtained from the particles that aligned to foreign references were of visibly lower image quality.

A third dataset with 3,852 images was recorded from a different grid of the activated sample. The images were aligned to the combined references and then classified. Numerical analysis showed a distribution similar to the original activated dataset and confirms the results.

\begin{tabular}{|c|c|c|c|c|c|c|c|}
\hline $\begin{array}{c}\mathbf{B}^{a c t} \\
\text { references }\end{array}$ & $\begin{array}{c}\text { Total } \\
\text { images }\end{array}$ & \# in class & $\%$ & \# in class & $\%$ & \# in class & $\%$ \\
\hline $\mathbf{B}^{a c t}$ & 7057 & 1092 & $15,47 \%$ & 484 & $6,86 \%$ & 430 & $6,09 \%$ \\
\hline $\mathrm{B}^{*}$ & 6780 & 383 & $5,65 \%$ & 123 & $1,81 \%$ & 194 & $2,86 \%$ \\
\hline$B^{*}(2)$ & 3852 & 220 & $5,71 \%$ & 83 & $2,15 \%$ & 135 & $3,50 \%$ \\
\hline $\mathbf{B}^{a c t}+3$ Cycles & 7057 & 779 & $11.04 \%$ & 473 & $6.70 \%$ & 454 & $6.43 \%$ \\
\hline$B^{*}+3$ Cycles & 6780 & 297 & $4.38 \%$ & 0 & $0.00 \%$ & 206 & $3.04 \%$ \\
\hline
\end{tabular}




\begin{tabular}{|c|c|c|c|c|c|c|c|}
\hline $\begin{array}{c}\mathbf{B}^{*} \\
\text { references }\end{array}$ & $\begin{array}{c}\text { Total } \\
\text { images }\end{array}$ & \# in class & $\%$ & \# in class & $\%$ & \# in class & $\%$ \\
\hline $\mathbf{B}^{a c t}$ & 7057 & 406 & $5,75 \%$ & 277 & $3,93 \%$ & 251 & $3,56 \%$ \\
\hline $\mathbf{B}^{*}$ & 6780 & 946 & $13,95 \%$ & 460 & $6,78 \%$ & 523 & $7,71 \%$ \\
\hline$B^{*}(2)$ & 3852 & 499 & $12,95 \%$ & 180 & $4,67 \%$ & 241 & $6,26 \%$ \\
\hline $\mathbf{B}^{a c t}+3$ Cycles & 7057 & 0 & $0.00 \%$ & 0 & $0.00 \%$ & 0 & $0.00 \%$ \\
\hline $\mathrm{B}^{*}+3$ Cycles & 6780 & 865 & $12.76 \%$ & 372 & $5.49 \%$ & 502 & $7.40 \%$ \\
\hline
\end{tabular}

a
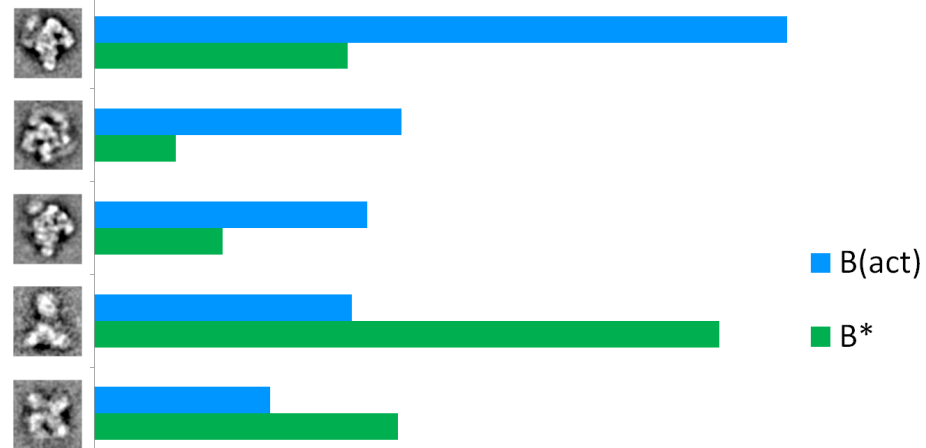

B
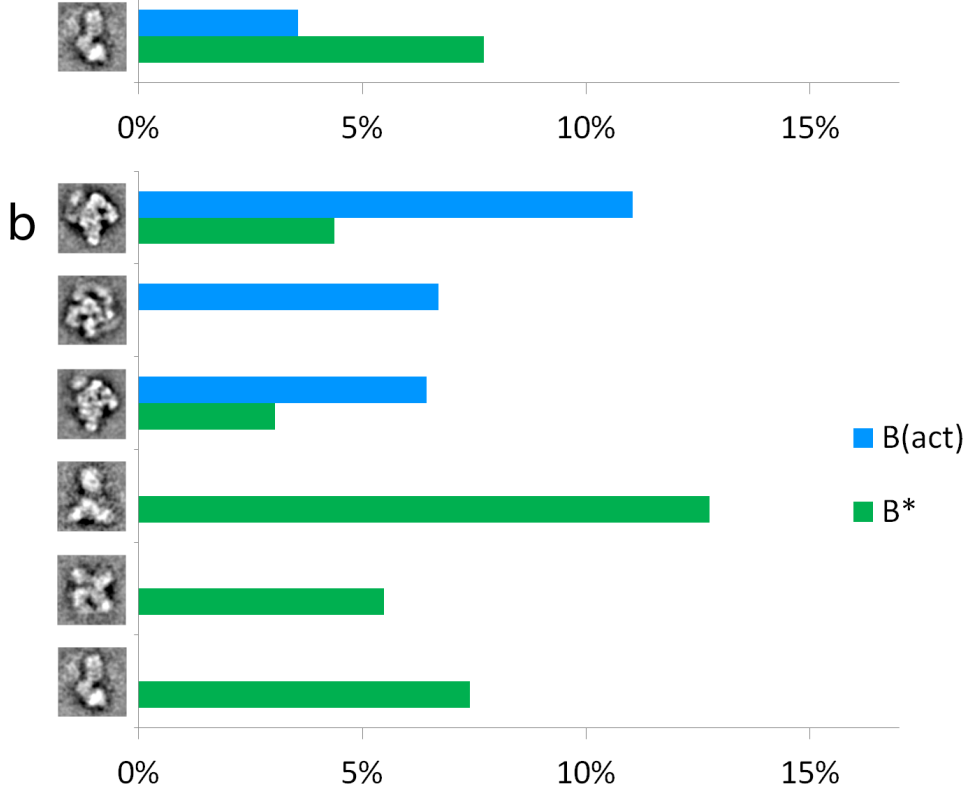

Figure 3.2: (a) Fraction of particles from the datasets aligning to the references after injection of bias. (b) Fraction of particles from the datasets aligning to the references after three additional cycles of alignment and classification. In each chart, the top three references are derived from the dataset before activation, the lower three from the dataset after activation. 


\section{Results}

Subsequently, the initial two datasets were subjected to three more rounds of independent refinement and the composition of the resulting class averages was analyzed again. Predictably, this caused the number of particles that aligned to foreign references to decrease to the point where the pre-activated sample showed no more foreign views at all. The activated sample, however, still included a percentage of particles that align to pre-activated views. This is consistent with experimental data that indicates a non-quantitative conversion upon Prp2 mediated activation [Warkocki et al., 2009].

\subsection{Chemical modification}

\subsubsection{GraFix Preparation}

It was examined whether chemical additives in the GraFix protocol [Kastner et al., 2008] could improve the fixation so that conformational heterogeneity in the sample is further limited. Several additives were used in the GraFix gradient along with the glutaraldehyde. Not only would the sample encounter a steadily increasing concentration of crosslinker, but also of the respective additive. The tested additives and their concentrations in the high density gradient were:

\begin{tabular}{ccc}
\hline & Additive & Concentration \\
\hline \hline A & unmodified & \\
B & Tantalum bromide, $\mathrm{Ta}_{6} \mathrm{Br}_{14}$ & $0.5 \mathrm{mM}$ \\
C & Potassium hexachloroplatinate, $\mathrm{K}_{2}\left[\mathrm{PtCl}_{6}\right]$ & $2.8 \mathrm{mM}$ \\
D & Sodium sulfate, $\mathrm{Na}_{2} \mathrm{SO}_{4}$ & $20 \mathrm{mM}$ \\
E & Triflouroethanol (TFE), $\mathrm{CF}_{3} \mathrm{CH}_{2} \mathrm{OH}$ & $30 \%[\mathrm{v} / \mathrm{v}]$ \\
F & Cadmium chloride, $\mathrm{CdCl}_{2}$ & $12 \mathrm{mM}$ \\
G & Polyethylene glycol (PEG) 4000 & $50 \mathrm{mM} \mathrm{(20 \% [v/v])}$ \\
H & Polyethylene glycol (PEG) 8000 & $25 \mathrm{mM}(20 \%[\mathrm{v} / \mathrm{v}])$ \\
\hline
\end{tabular}

The additives were chosen for their reported effects in crystallography $(B, C, F)[M c P h e r s o n$ and Cudney, 2006], protein stabilizing (E, G, H) [Shiraki et al., 1995, McPherson, 1985] or cosmotropic properties (D) [Ramos and Baldwin, 2002]. During automated fractionation, the PEG-modified gradients showed a reduced flow rate, resulting in $20 \%$ more fractions and a relative shift of the 
sample peak towards the lighter fractions. The TFE-modified gradient even resulted in double the amount of usual fractions. Its relative peak position was largely unaffected. (see Fig. 3.3 on page 39)

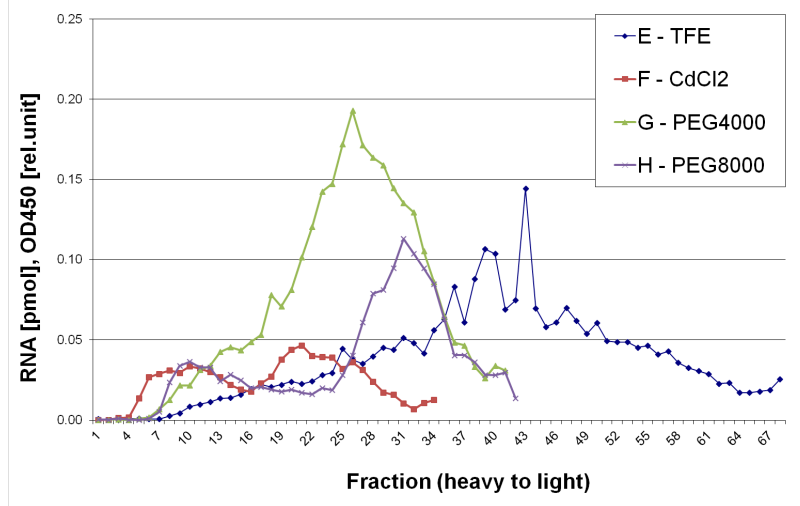

Figure 3.3: Fraction distribution after modified GraFix for conditions E-H.

\subsubsection{Image aquisition}

Of the tested conditions (shown in Fig. 3.4 on the following page), only A-D provided usable image data. Conditions E-H actually had an adverse effect on the preparation: TFE (E) led to severe sample aggregation, $\mathrm{CdCl}_{2}(\mathrm{~F})$ caused the particles to degenerate and both PEG samples $(G, H)$ showed degraded, sparsely adsorbed particles with low contrast (with the observation being even more prominent in $\mathrm{H})$.

\subsubsection{Image Processing}

From the usable conditions, four datasets were recorded and analyzed. From each dataset 5,500 images $\left(4,633\right.$ for $\left.\mathrm{Na}_{2} \mathrm{SO}_{4}\right)$ were used for independent reference-free alignment with subsequent multivariate statistical analysis and classification [van Heel, 1984]. The resulting class averages were used as a basis for reference images in the next cycle of alignment. Each dataset was subjected to six rounds of alignment and classification. The class averages of condition B showed a visibly higher contrast (see figure 3.5 on page 41 ).

All datasets showed the same dominant view to which a large number of particles aligned. These class averages were extracted and their contributing single images counted to determine the percentage of particles that align to the main view (see table on page 41). 

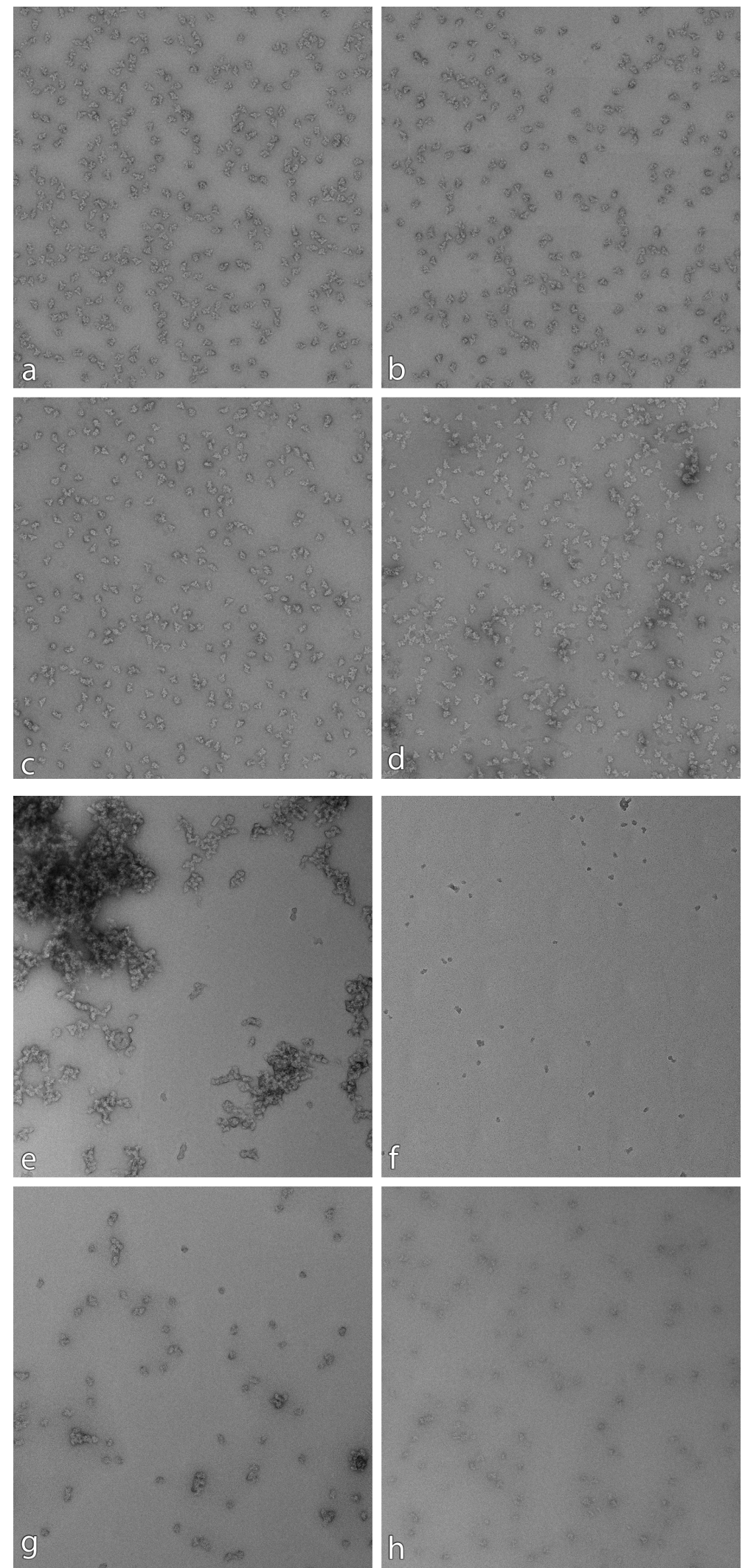

Figure 3.4: Electron micrographs of the spliceosomal B-complex, modified by (a) unmodified GraFix, or modified with (b) $\mathrm{Ta}_{6} \mathrm{Br}_{14}$, (c) $\mathrm{K}_{2}\left[\mathrm{PtCl}_{6}\right]$, (d) $\mathrm{Na}_{2} \mathrm{SO}_{4}$, (e) TFE (f) $\mathrm{CdCl}_{2}$, (g) PEG 4000, (h) PEG 8000. (e) shows the aggregates that were typical for the sample, (f, $\mathrm{g}$, h) show low particle density. Particles in (f) show strong degeneration, while ( $\mathrm{g}, \mathrm{h}$ ) show low contrast and partial degradation. 


\begin{tabular}{cc|ccc}
\hline \multicolumn{2}{c|}{ Condition } & Images aligning to main view & Size of dataset & Percentage \\
\hline \hline A & Unmodified & 1918 & 5500 & $35 \%$ \\
B & $\mathrm{Ta}_{6} \mathrm{Br}_{14}$ & 2242 & 5500 & $41 \%$ \\
C & $\mathrm{K}_{2}\left[\mathrm{PtCl}_{6}\right]$ & 1349 & 5500 & $25 \%$ \\
D & $\mathrm{Na}_{2} \mathrm{SO}_{4}$ & 1276 & 4633 & $28 \%$ \\
\hline
\end{tabular}

The data suggests that condition B causes the particles to adopt a preferred orientation on the carbon foil, while C and D promote more a varied arrangement. For samples like the spliceosome, which tend to bind in a highly preferred orientation, it is actually desirable to have the particle adopt a greater variety of orientations on the grid surface.
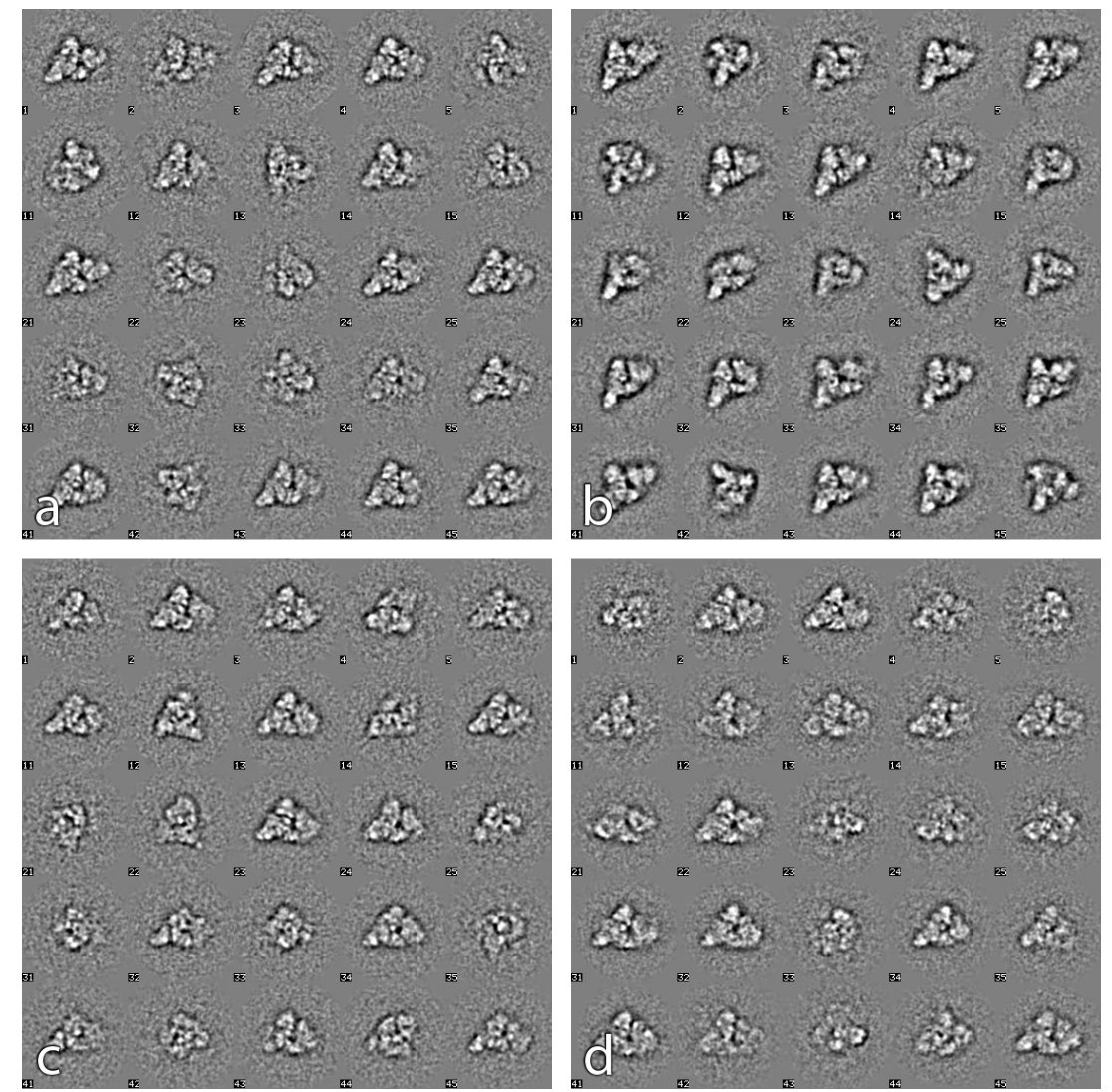

Figure 3.5: Typical class averages of the conditions (a) unmodified GraFix, (b) $\mathrm{Ta}_{6} \mathrm{Br}_{14}$, (c) $\mathrm{K}_{2}\left[\mathrm{PtCl}_{6}\right]$, (d) $\mathrm{Na}_{2} \mathrm{SO}_{4}$. No significant differences are visible, except for an increased contrast of condition (b). 

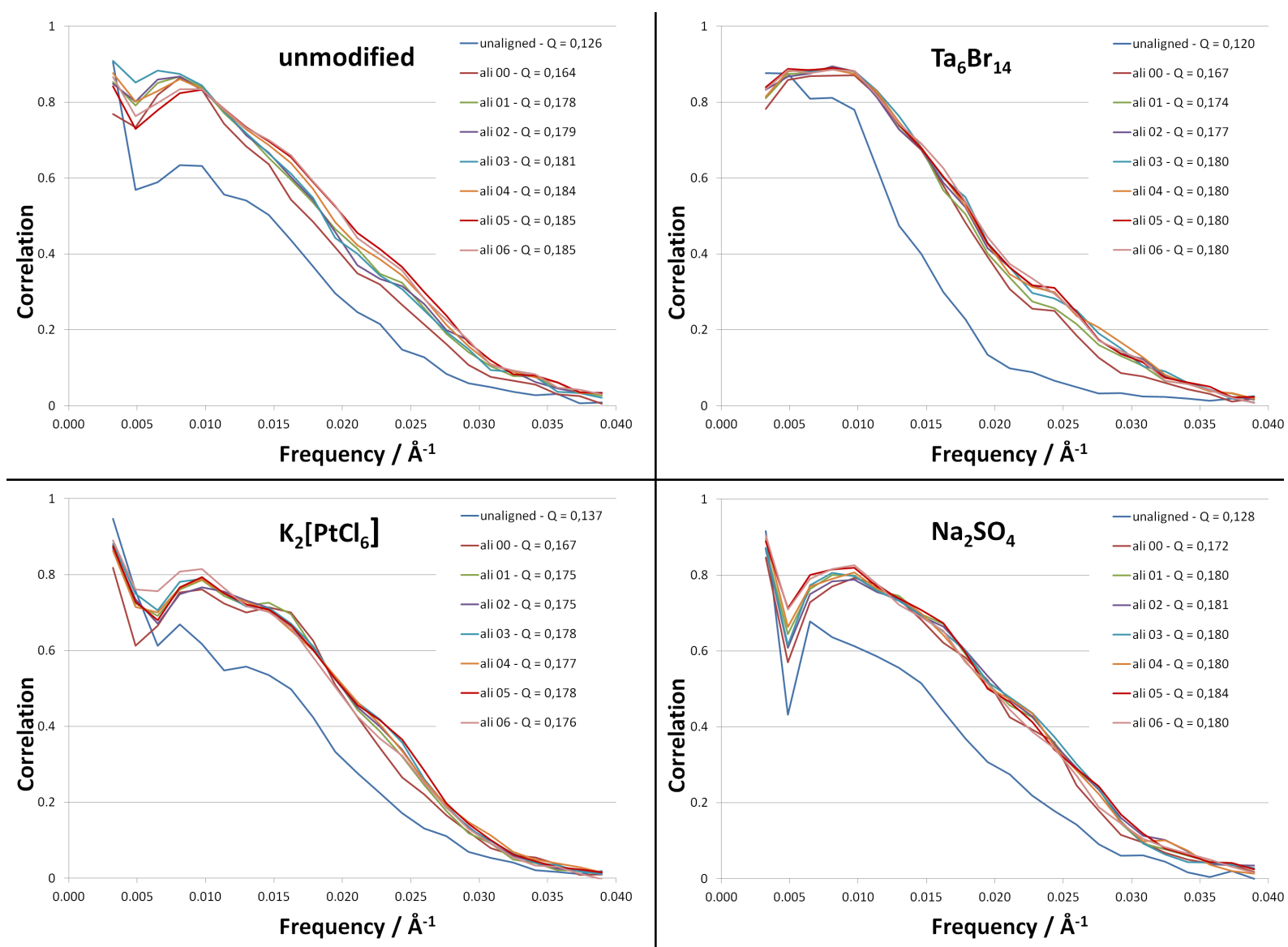

Figure 3.6: FRC plots after various cycles of alignment and classification. Correlation improves significantly upon alignment compared to the unaligned original dataset. Further cycles gradually improve correlation for each condition, mainly in the $0.01-0.03 \AA^{-1}$ region.

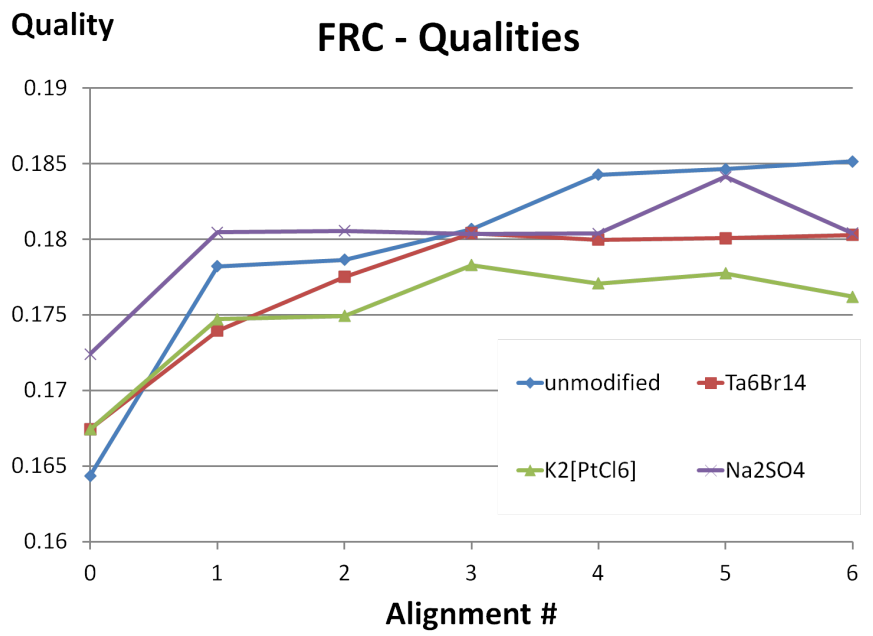

Figure 3.7: Quality of the FRC over multiple cycles of alignment. After an initial jump, quality increases gradually and stabilizes with successive alignments. 


\subsubsection{Fourier Ring Correlation}

To analyze the homogeneity of the datasets, Fourier-ring-correlation (FRC) analysis was used. FRC is a similarity measure between two images by a correlation in Fourier space [van Heel, 1987b]. For each analysis, the input data was classified with the CowCluster algorithm [in-house software, programmed by Mario Lüttich] and 150 class averages were generated. The images that comprise each class average were extracted into a separate file and there divided into two stacks. Each stack was then again averaged so that in the end each file contained two images, each of which comprised half the images that made up one class average. The two subaverages were then compared via Fourier-ring-correlation to determine their similarity. Higher correlation means that the images that make up the class average are more homogeneous, which generally signifies a more homogeneous dataset. The FRC was calculated for each class average, added up into one combined plot and normalized. The ClassTest script and program [in-house software, programmed by Mario Lüttich] were used to largely automate the process. In each dataset, the unaligned images as well as the images after each round of alignment were used as an input for the analysis. The correlation was plotted against spatial frequency (see Fig. 3.6 on the preceding page).

The integral of the plot between the spatial frequencies $\mathrm{x}=0.0032 \AA^{-1}$ and $\mathrm{x}=0.0390 \AA^{-1}$ served as an index for the general correlation and therefore the quality of the dataset. For the calculation of the integral, the first two data points $\left(\mathrm{x}<0.0032 \AA^{-1}\right)$ were omitted because the data for $\mathrm{x}=0$ varied erratically between 0 and 1 and would distort the integral. Data for $\mathrm{x}>0.04 \AA^{-1}$ was omitted for lack of relevance. The quality-index of the dataset increases significantly after the first alignment and then increases slightly over subsequent alignments with the unmodified condition gaining the most in the process.

A comparison of the plots after six alignments shows an increased correlation in the 0.005 $0.015 \AA^{-1}$ region for condition B (see figure 3.8 on the following page). Since these lower spatial frequencies are on the range of 60 - $200 \AA$ detail, this coincides with the observed higher contrast of the particles in this preparation.

To evaluate the relevance of these differences in quality, a second dataset of 3,500 images was recorded and analyzed for conditions A and B. It was then compared to a subset of 3,500 images from the original datasets. While both curves for the unmodified condition progressed similarly, 


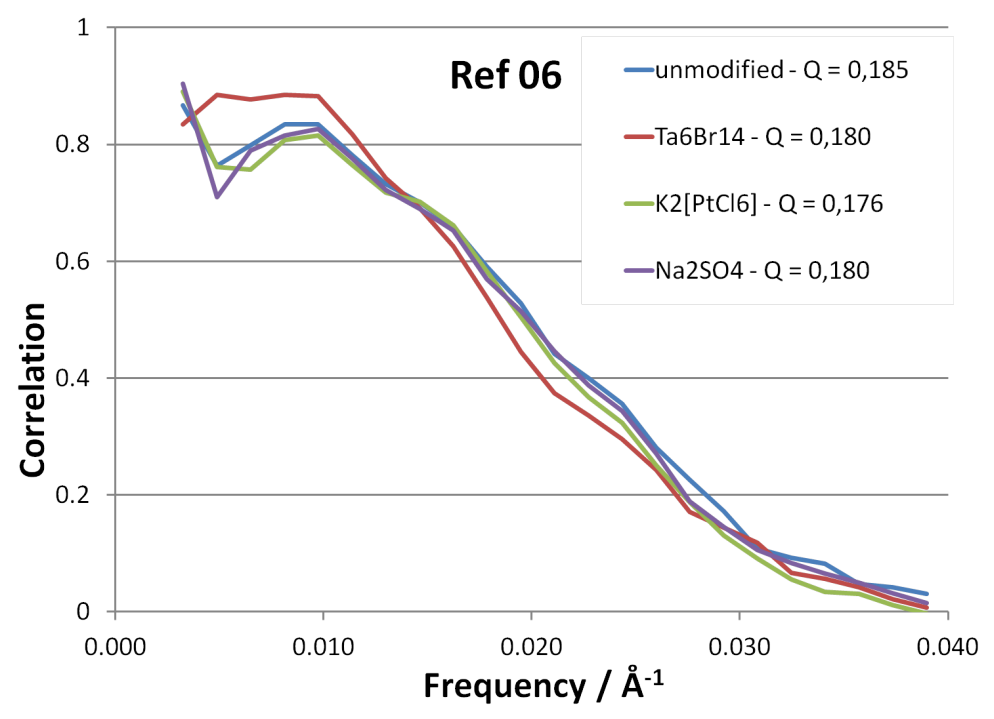

Figure 3.8: FRC plot of the 4 conditions after 6 cycles of alignment and classification. The graph for $\mathrm{Ta}_{6} \mathrm{Br}_{14}$ shows a noticeable increased correlation between $0.005-0.015 \AA^{-1}$, while correlation is lower between $0.015-0.028 \AA^{-1}$ compared to the other conditions.

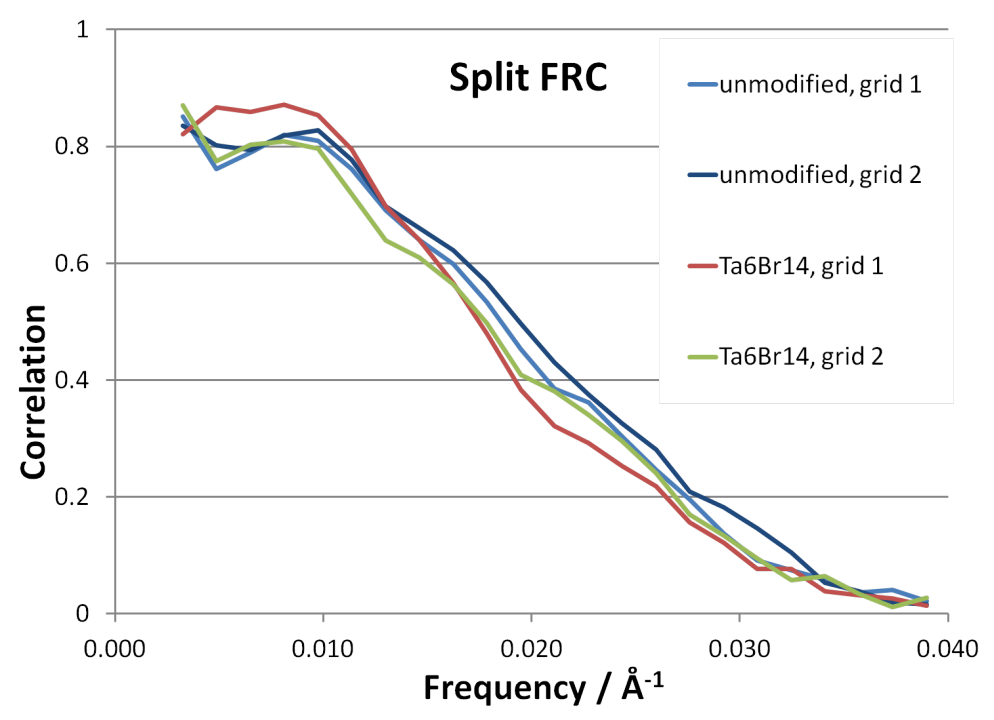

Figure 3.9: FRC of the first and second dataset for conditions $A$ and $B$. Noticeable is the absence of the increased correlation between $0.005-0.015 \AA^{-1}$ in the graph for $\mathrm{Ta}_{6} \mathrm{Br}_{14}$, grid 2 . 
the second $\mathrm{Ta}_{6} \mathrm{Br}_{14}$-dataset lacked the previously noticed increased correlation in the 0.005 $0.015 \AA^{-1}$ region. Instead it even showed a reduced correlation in that region (see figure 3.9 on the preceding page). To investigate this discrepance further, each set of 3,500 images was split into two subsets of 1,750 images by randomised sorting. Again, FRC-analysis via ClassTest was used to determine the homogeneity of the data.

\begin{tabular}{c|cccc}
\hline & \multicolumn{2}{|c}{ A (unmodified) } & \multicolumn{2}{c}{$\mathbf{B}\left(\mathbf{T a}_{6} \mathbf{B r}_{14}\right)$} \\
& Original dataset & Second dataset & Original dataset & Second dataset \\
\hline \hline Full set & 0.172 & 0.180 & 0.169 & 0.167 \\
Subset 1 & 0.166 & 0.174 & 0.165 & 0.165 \\
Subset 2 & 0.167 & 0.177 & 0.169 & 0.160 \\
Standard deviation & 0.004 & 0.003 & 0.003 & 0.003 \\
\hline
\end{tabular}

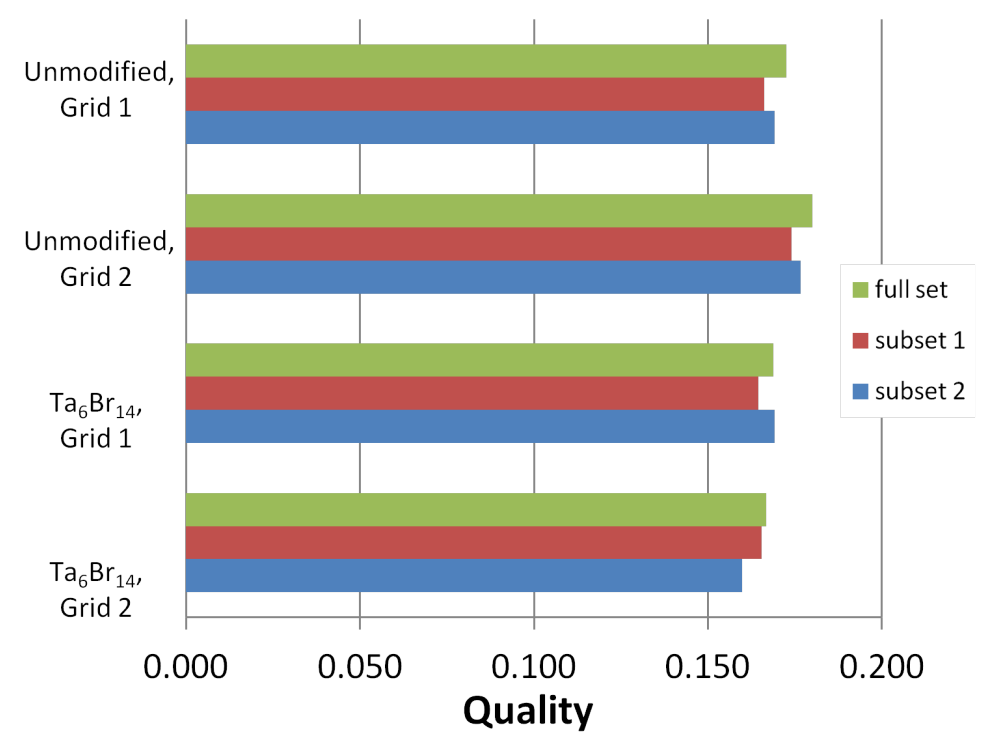

Figure 3.10: Bar chart showing the qualities of the validation datasets, calculated as the integral of the FRC-plot.

The values of the quality-indices are well within range of two standard deviations. This means that the differences between different fixation conditions are on the same order of magnitude as the variance observed from recording and picking between two datasets of the same condition or even the subsets. This implies that the originally noticed increase in correlation for condition $\mathrm{B}$ was an outlier. 


\section{Results}

According to the statistical data, the preparation additives show no significant effect. If at all, the tested modifications seem to affect sample homogeneity rather negatively compared to the unmodified sample.

\subsection{Cryo-Fixation}

Fischer et al. [2010] have examined the temperature-dependence of ribosome dynamics and have shown that the rotation of the ribosomal subunits relative to each other is more pronounced at higher temperatures. This leads to increased heterogeneity in the data, which could be visualized in reconstructions (see figure 4.1 on page 60) and analyzed quantitatively. At $4{ }^{\circ} \mathrm{C}$ there is a distinct preferred rotation angle that the $30 \mathrm{~S}$ subunit adopted relative to the $50 \mathrm{~S}$ subunit. This distribution broadens with increasing temperatures until at $37^{\circ} \mathrm{C}$ the thermal energy is sufficient for the subunit to rotate with almost no preferred rotation angle. The aim of this investigation was to examine whether this effect could be extended into the other direction by preparing samples at temperatures below $0{ }^{\circ} \mathrm{C}$ and stabilizing the particles in a thermodynamically preferred conformation adopted at these lowered temperatures in order to further limit heterogeneity.

After modification by a service technician, the Ultracentrifuges could be set to temperatures as low as $-9.9^{\circ} \mathrm{C}$, which was the temperature used for the cryo-fixation experiments. Actual temperature measurements with an electronic thermometer inside the gradients after centrifugation showed $-6^{\circ} \mathrm{C}$. However, during the measurement the gradients were already warming rapidly since they had been removed from the centrifuge.

\subsubsection{Adapting the GraFix protocol for cryo-fixation}

Three factors had to be considered when carrying out a GraFix fixation at cryo conditions: Glutaraldehyde activity, centrifuge speed and gradient freezing. The concentration of glutaraldehyde had to be increased in order to compensate for the reduced activity at lower temperatures. At the same time, the gradient would be more viscous, so the centrifuge speed had to be increased in order to achieve a comparable migration of the sample without increasing the time of the centrifugation run. Finally, at temperatures below $0{ }^{\circ} \mathrm{C}$, there is the risk of the 
gradients freezing.

\section{Glutaraldehyde activity}

Ulrich Steuerwald and Jürgen Wawrzinek have developed a gel-screen for the glutaraldehyde fixation rate on a sample [Chari et al., 2012]. The migration of apoferritin or glutamate dehydrogenase in a native PAGE gel corresponds directly with the glutaraldehyde concentration the respective sample encountered in the gradient and therefore the grade of fixation it underwent.

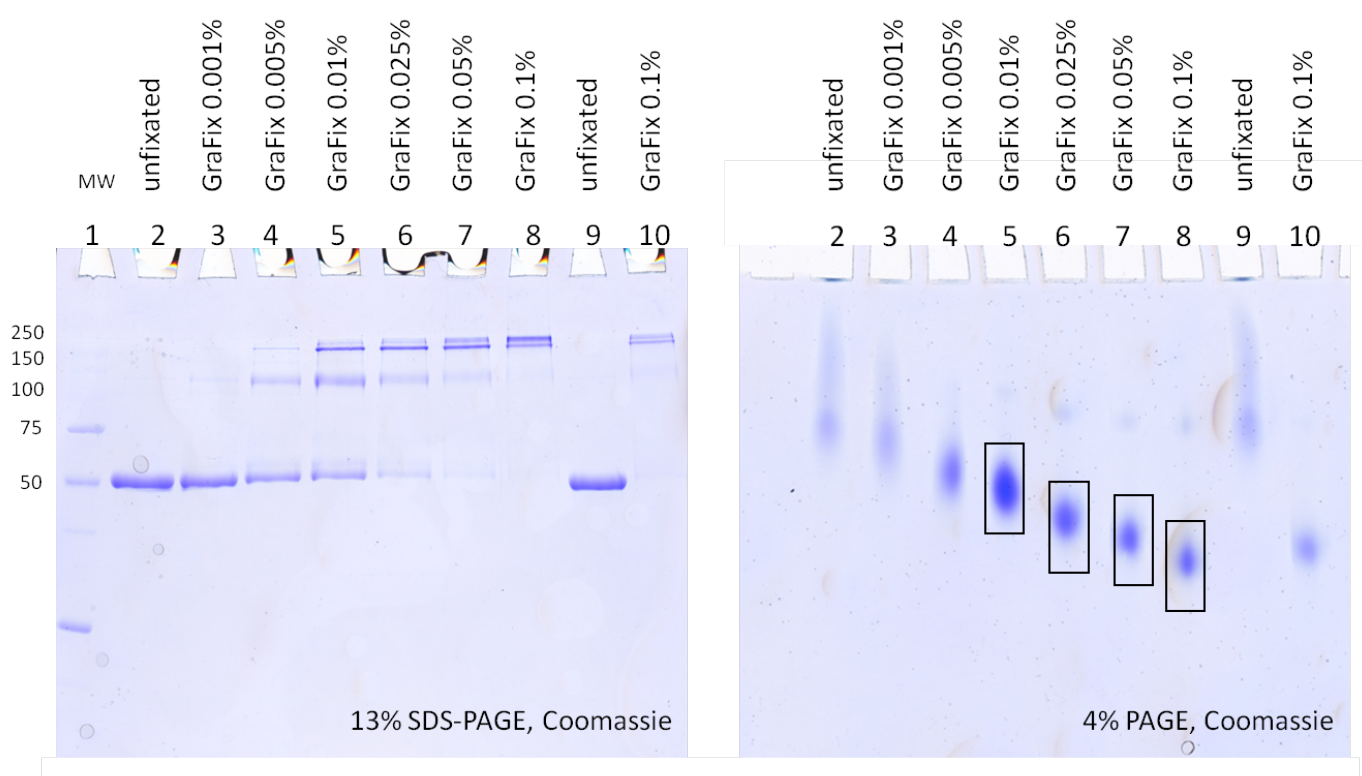

Figure 3.11: SDS- (left) and native PAGE (right) gels of GDA fixated at $4{ }^{\circ} \mathrm{C}$ with different concentrations of glutaraldehyde. Increased glutaraldehyde concentration leads to stronger migration in the native gel. Image courtesy of Ulrich Steuerwald.

By using glutamate dehydrogenase as a model system, different glutaraldehyde concentrations were used for cryo-fixation at $-9.9{ }^{\circ} \mathrm{C}$ and their migration behaviour compared to that of samples fixated at $+4{ }^{\circ} \mathrm{C}$ (see Fig. 3.12 on the next page). The data shows clearly that fixation occurs at the lowered temperature but, as expected, with greatly reduced activity. In a second experiment, several glutaraldehyde concentrations at cryo-fixation conditions were tested against $0.1 \%$ at $+4{ }^{\circ} \mathrm{C}$, the usual standard condition (see Fig. 3.13 on the following page). Migration of the standard lies between the cryo-fixated samples with $0.8 \%$ and $1.5 \%$ glutaraldehyde. From these results, an eight- to tenfold increase of crosslinker for cryo-fixation is recommended for future experiments. 


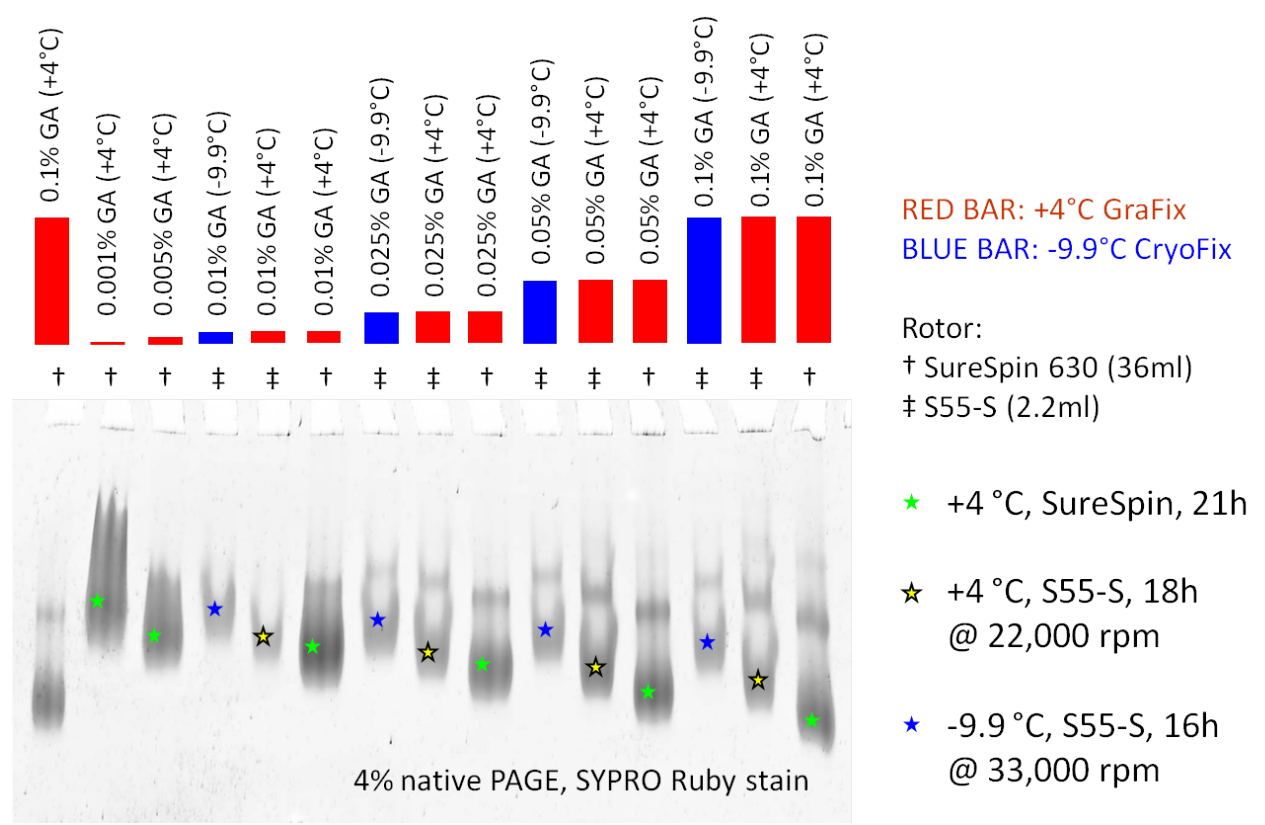

Figure 3.12: Comparison of early cryo-fixation with regular GraFix-conditions. Bar length is proportional to glutaraldehyde concentration. Increased glutaraldehyde fixation leads to stronger migration. The migration pattern for the cryo-fixated sample (blue bars and stars) is not as distinct as the pattern at the same concentration, but at $4{ }^{\circ} \mathrm{C}$ (yellow stars), implying less fixation due to the reduced temperatures. The green stars refer to an initial run in a larger rotor and is not relevant for this comparison.

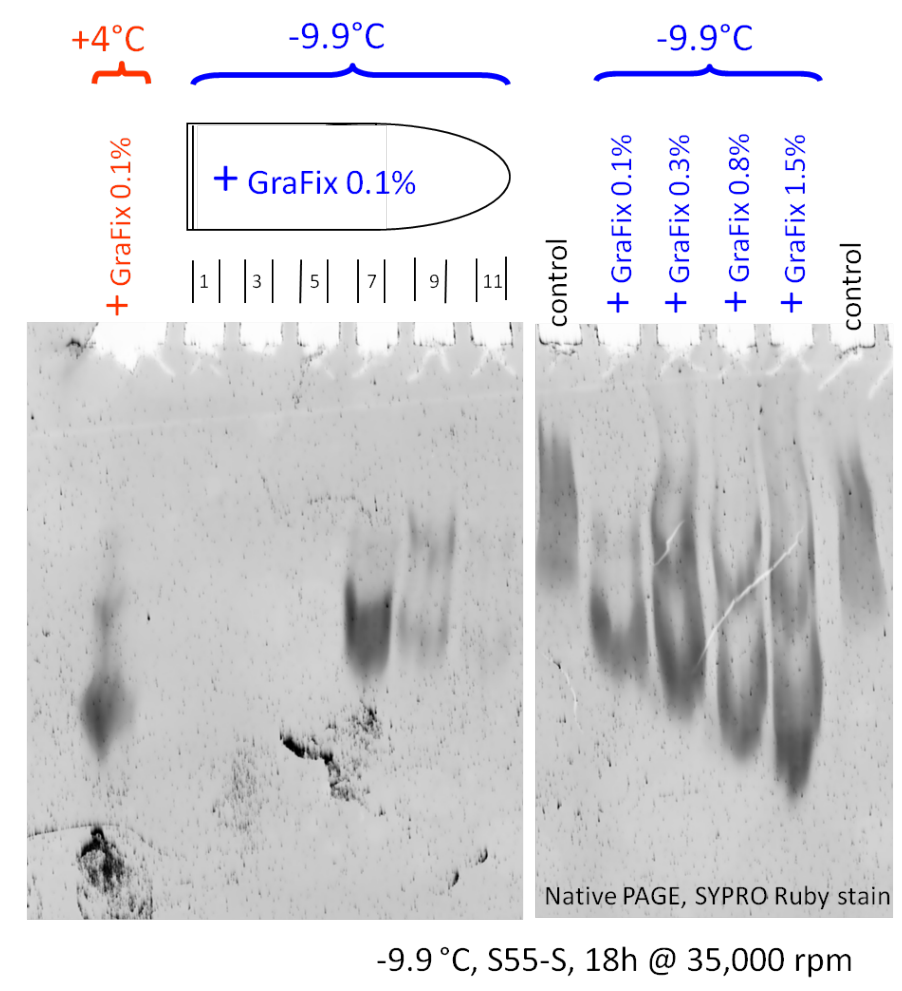

Figure 3.13: Native PAGE gel of cryo-fixated GDA. Standard GraFix control in lane 1, gradient fraction analysis, unfixated control and $0.1 \%-1.5 \%$ glutaraldehyde samples. The effect from inceased fixation under cryo-conditions is clearly visible. Migration of the $0.1 \%$ standard condition at $4{ }^{\circ} \mathrm{C}$ lies between $0.8 \%$ and $1.5 \%$ of the cryo-fixated sample. 


\section{Centrifuge speed}

The required rotations per minute were initially estimated with the help of the Compass run simulation software. Due to the software not being designed for negative temperature conditions (the lowest temperature that can be simulated is $0{ }^{\circ} \mathrm{C}$ ), the predictions were just an educated extrapolation and proved inadequate. The centrifugation conditions had to be optimized by experiment to attain sufficient migration of the sample into the gradient. For glutamate dehydrogenase in a 5-20\% glycerol gradient, a 50\% increase in required rpm proved suitable. The viscosity of the $10-40 \%$ sucrose gradient for the ribosomes did not increase as much and initial experiments suggested an increase of $35 \%$ in rpm. However, to avoid the possibility of the subunits disassociating under high pressure [Infante and Baierlein, 1971], the rpm were only increased by $10 \%$ and instead a longer runtime was chosen to compensate partially.

\section{Gradient freezing}

Usually, gradient freezing was not a problem. Occasionally, a gradient would be found half frozen in the rotor after centrifugation. When thawed and harvested, those gradients showed a uniform distribution of sample in the bottom (i.e. unfrozen) fractions, suggesting a top-down freezing process that stirred the gradient. This occasional and unpredictable event was rare enough that it did not receive further attention.

\subsubsection{Validation with the 70 S ribosome}

With the results obtained from the model system glutamate dehydrogenase, the 70S ribosome was selected for a proof of principle. As a measure for the effects of cryo-fixation, the intersubunit rotation between its $50 \mathrm{~S}$ and $30 \mathrm{~S}$ subunits was examined. A homogeneous sample should exhibit a distinct peak at a preferred rotation angle, whereas a dynamic, heterogeneous sample would show a broader distribution of rotation angles. To see whether GraFix and Cryo-fixation could affect and stabilize the conformational distribution of the particle and retain it upon subsequent temperature changes, the samples were prepared for cryo-EM at two different temperatures. The standard ambient temperature for vitrification is $4{ }^{\circ} \mathrm{C}$, which is also the lowest possible setting for the Vitrobot. Therefore, cryo-fixated gradients will have to be rewarmed after centrifugation. 


\section{Results}

The second selected preparation temperature was $20^{\circ} \mathrm{C}$, to better compare the effects of increased temperature on the fixation.

\section{CryoFix and GraFix preparation}

Six conditions were examined: unfixated (control), GraFix treated $\left(+4{ }^{\circ} \mathrm{C}, 0.15 \%\right.$ glutaraldehyde) and Cryo-fixated $\left(-9.9{ }^{\circ} \mathrm{C}, 1 \%\right.$ glutaraldehyde) samples were prepared for cryo-EM at $4{ }^{\circ} \mathrm{C}$ and $20{ }^{\circ} \mathrm{C}$ respectively.

\begin{tabular}{ccc}
\hline Sample name & Centrifuge temperature & Vitrobot temperature \\
\hline \hline P17 & No GraFix & $4{ }^{\circ} \mathrm{C}$ \\
P10 & No GraFix & $20^{\circ} \mathrm{C}$ \\
P12 & $4{ }^{\circ} \mathrm{C}$ & $4{ }^{\circ} \mathrm{C}$ \\
P11 & $4{ }^{\circ} \mathrm{C}$ & $20^{\circ} \mathrm{C}$ \\
P15 & $-9.9{ }^{\circ} \mathrm{C}$ & $4{ }^{\circ} \mathrm{C}$ \\
P16 & $-9.9{ }^{\circ} \mathrm{C}$ & $20^{\circ} \mathrm{C}$ \\
\hline
\end{tabular}

No freezing of the cryo-gradients was observed. To verify the migration of the sample, the gradient fractions were analyzed in a Nanodrop spectrophotometer. Optical density measurement is disturbed by the presence of glutaraldehyde, as it absorbs in the same range as the ribosome [Kawahara et al., 1992, Sperrazza et al., 1980]. Therefore, for each condition one gradient without glutaraldehyde was run in the centrifuge and analyzed to identify the sample fractions. The GraFix gradient showed the sample peak in fractions 10 and 11 of 23 fractions (with a dead volume of 6 fractions from the fractionator), which translates to $75 \%$ migration. The CryoFix gradient showed the sample peak in fractions 13 and 14 of 24 fractions, signifying a migration of $60 \%$. Particle density on the grids of the cryo-fixated samples was observed as very low, despite the increased adsorption times. Images of GraFix treated or unfixated samples showed on average 50-70 particles per image, whereas the cryo-fixated samples only yielded around 15-20 particles per image. The reason for this is still unkown. Loading the double amount of sample remedies this (Ashwin Chari, personal communication). 


\section{Supervised Classification}

To examine the effect of cryo-fixation on a heterogeneous sample, the 70S ribosome was selected as a model system. The angular distribution of the intersubunit rotation between the $30 \mathrm{~S}$ and $50 S$ subunits could serve as an indicator for heterogeneity. Earlier studies in our lab have reconstructed the $70 S$ ribosome in multiple states of subunit rotation [Fischer et al., 2010]. These structures allowed the projection of references for 2D alignment. 19 such reference-sets were generated with rotation angles from $-20^{\circ}$ to $+25^{\circ}$ (with $0^{\circ}$ being equivalent to the rotational state in the crystal structure) and a stepsize of $2.5^{\circ}$. Furthermore, one reference set was generated from a structure of the $50 \mathrm{~S}$ large subunit. This was done to sort out free $50 \mathrm{~S}$ subunits, which would otherwise disturb the analysis of intersubunit angular orientation. Each image from a dataset was then aligned to all 20 reference sets and assigned to the reference set with which it showed the best cross-correlation and therefore to the respective rotation angle. The angular distribution, along with the number of images that aligned to the $50 \mathrm{~S}$ reference, the total number of images and the percentage of $50 \mathrm{~S}$ particles is shown in Table 3.1 on page 55. For the analysis, only the angular range from $-12.5^{\circ}$ to $+17.5^{\circ}$ was used, because reconstructions from subgroups sorted outside of this range have yielded scattered densities for the 305 body, which is a sign for bad particle quality. Table 3.2 on page 55 shows the fraction of images with the respective angles along with the total number of particles inside the angular range. Figures 3.14 and 3.15 on the next page show plots of the angular distribution of samples vitrified in the Vitrobot at ambient temperatures of $4{ }^{\circ} \mathrm{C}$ and $20^{\circ} \mathrm{C}$, respectively. The $4{ }^{\circ} \mathrm{C}$ graphs (Fig. 3.14) show the unfixated sample with a distinct peak at $0^{\circ}$ rotation, a secondary peak at $5^{\circ}$ and a tertiary peak at $-5^{\circ}$. Table 3.3 on page 55 shows the proportion of the $0^{\circ}$ peak compared to the $5^{\circ}$ and $-5^{\circ}$ peaks. Application of GraFix at $4{ }^{\circ} \mathrm{C}$ narrows the angular distribution, increasing the primary peak while reducing the secondary one. However, the tertiary peak is enlarged. The $-9.9^{\circ} \mathrm{C}$ CryoFix sample continues this trend, slightly increasing the main and tertiary peaks further while decreasing the secondary one. The $20^{\circ} \mathrm{C}$ graphs (Fig. 3.15) show the unfixated sample with a wide angular distribution, indicating a heterogeneous dataset, with the main and secondary peak almost equal in intensity. Stabilizing the sample with GraFix at $4{ }^{\circ} \mathrm{C}$ before warming to $20^{\circ} \mathrm{C}$ for the preparation significantly increases the main peak. While the secondary peak even increases slightly, the ratio between the $0^{\circ}$ and $5^{\circ}$ peaks increases from 1.08 to 1.27 . 


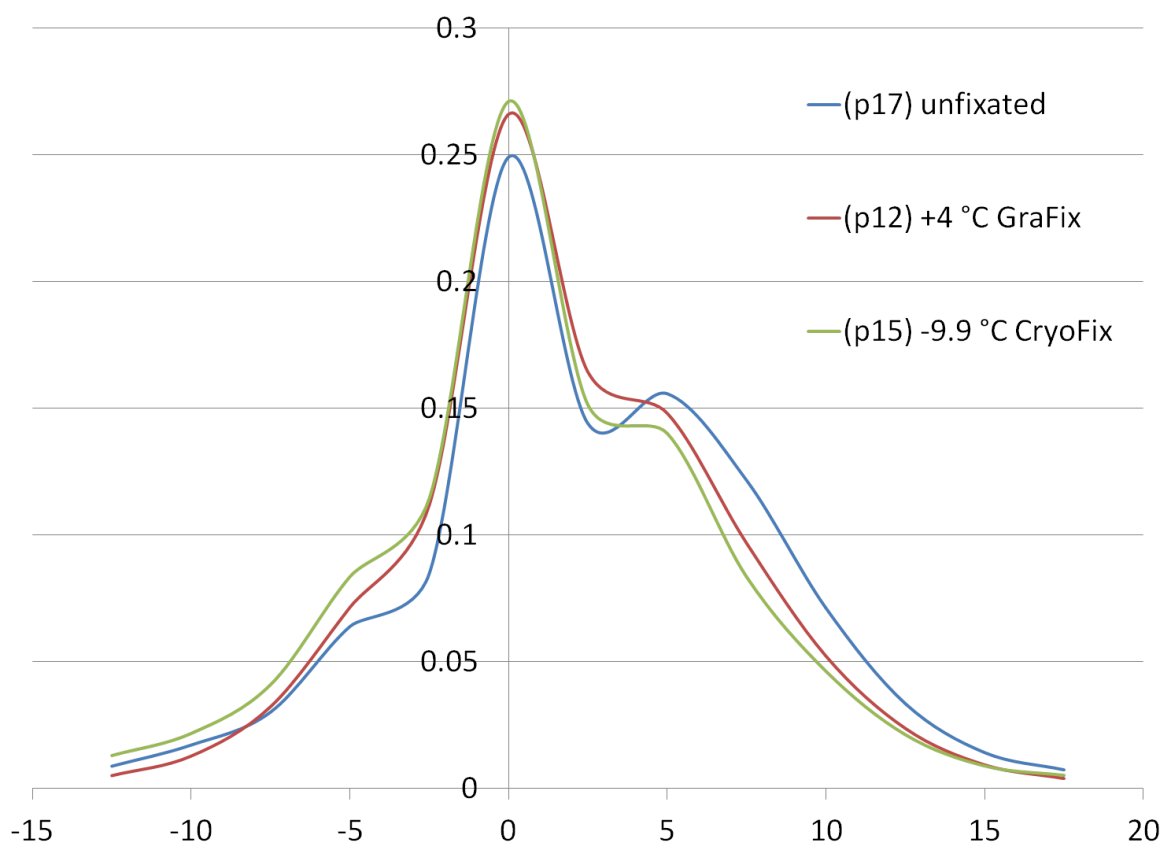

Figure 3.14: Angular distribution of ribosomal subunit rotation at $4{ }^{\circ} \mathrm{C}$ preparation temperature. GraFix treatment increases the population of the dominant configuration at $0^{\circ}$, while decreasing the $5^{\circ}$ population and slightly increasing the $-5^{\circ}$ population. Cryo-fixation increases this effect.

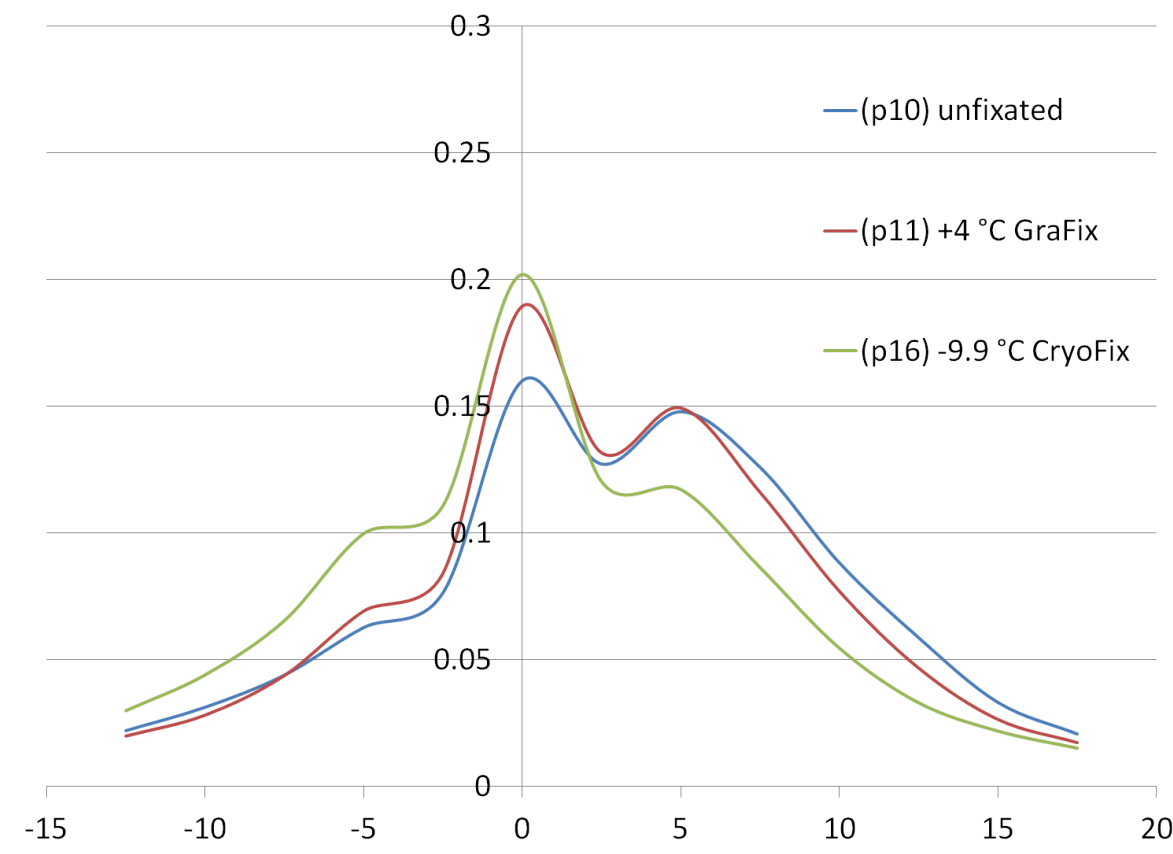

Figure 3.15: Angular distribution of ribosomal subunit rotation at $20^{\circ} \mathrm{C}$ preparation temperature. GraFix treatment increases the population at $0^{\circ}$, making it dominant in comparison with the $5^{\circ}$ population and also slightly increasing the $-5^{\circ}$ population. Cryo-fixation increases this effect significantly. 
Cryo-fixation at $-9.9{ }^{\circ} \mathrm{C}$ prior to $20^{\circ} \mathrm{C}$ preparation further stabilizes the main conformation and has a surprisingly drastic effect on the $+5^{\circ}$ and $-5^{\circ}$ side peaks, but again continues the trend. The data suggests a steadily increasing conformational stability of the datasets that culminates in the cryo-fixated condition prepared at $4{ }^{\circ} \mathrm{C}$ as the most homogeneous.

To evaluate the significance of the data, each dataset was split into three random subsets by extracting the correlation information from the already aligned images and sorting it into the first subset for images with the numbers (3n), into the second for images with the numbers $(3 n+1)$ and into the third subset for images with the numbers $(3 n+2)$. The relative angular distribution of the subset was then plotted and compared to its parent set (see figure 3.16 on the next page). From this, the standard deviation and relative standard deviation could be calculated. Tables 3.4 and 3.5 on page 56 show the numerical values. Already at first glance it can be noticed that the graphs of the series of $20^{\circ} \mathrm{C}$ preparations show a greater variance than their $4{ }^{\circ} \mathrm{C}$ preparation counterparts, which have their most noticeable divergence on the $5^{\circ}$ data point. In both data series, it seems like the relative standard deviation in the $-5^{\circ}$ to $5^{\circ}$ region decreases from unfixated over GraFix-treated to cryo-fixated conditions - which is consistent with a reduction of the visible variance of the graphs. The increase of the main peak was statistically significant (i.e. more than two standard deviations from each data point apart) in all cases except for the cryo-fixated $4{ }^{\circ} \mathrm{C}$ preparation, where only a slight increase could be observed. Still, the general trend indicates that the peak increase is consistent with the change in conditions from unfixated to cryo-fixated sample and suggests a narrowing of the conformational distribution towards a more homogeneous dataset. 

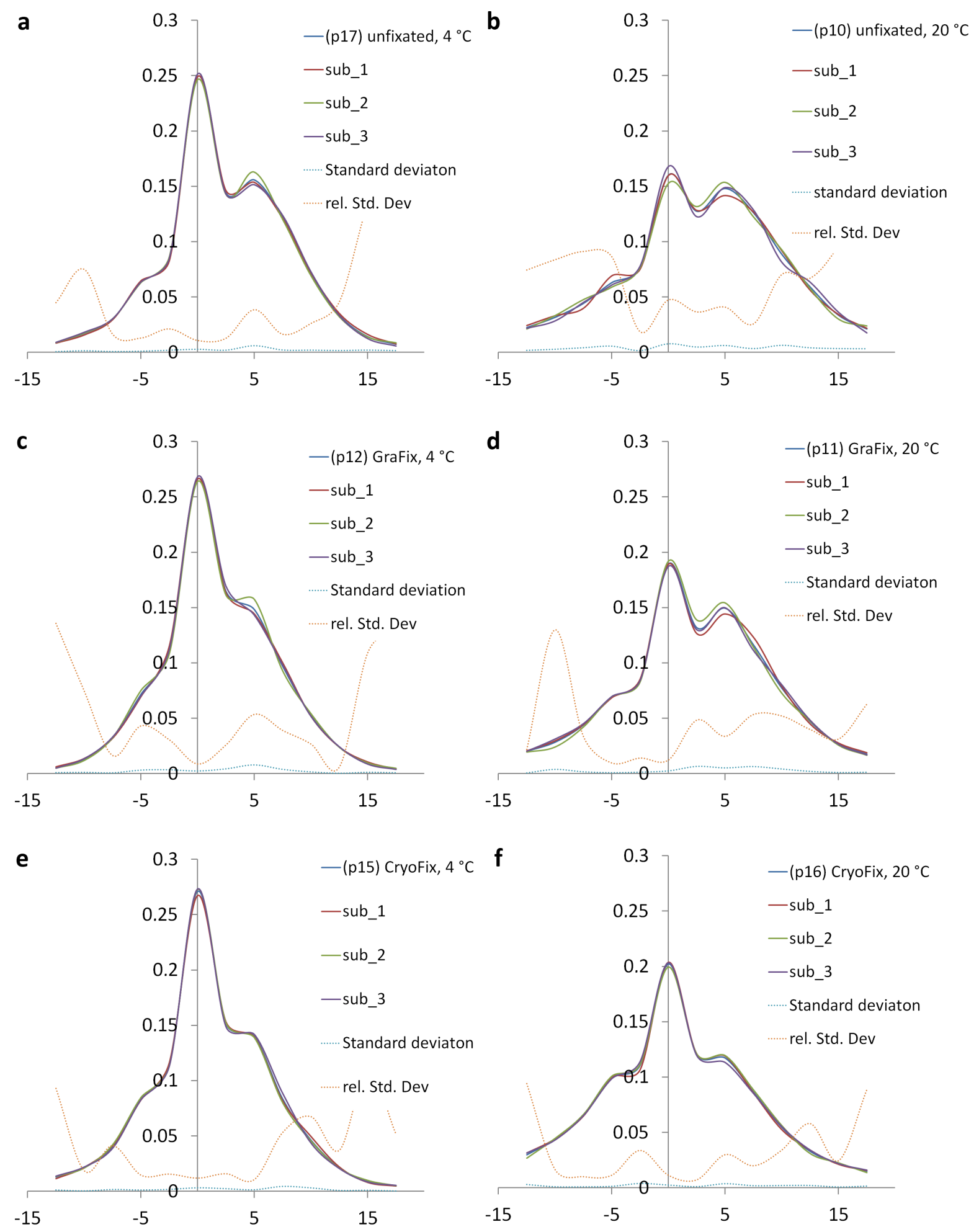

Figure 3.16: Angular distribution for all conditions including the split into three subsets. Standard deviation and relative standard deviation added as dotted lines. $(a, b)$ are the unfixated controls, $(\mathrm{c}, \mathrm{d})$ were fixated at $4{ }^{\circ} \mathrm{C}$, (e, f) were fixated at $-9.9^{\circ} \mathrm{C}$. Ambient temperature for cryo-EM preparation was $4{ }^{\circ} \mathrm{C}(\mathrm{a}, \mathrm{c}, \mathrm{e})$ or $20^{\circ} \mathrm{C}(\mathrm{b}, \mathrm{d}, \mathrm{f})$. 


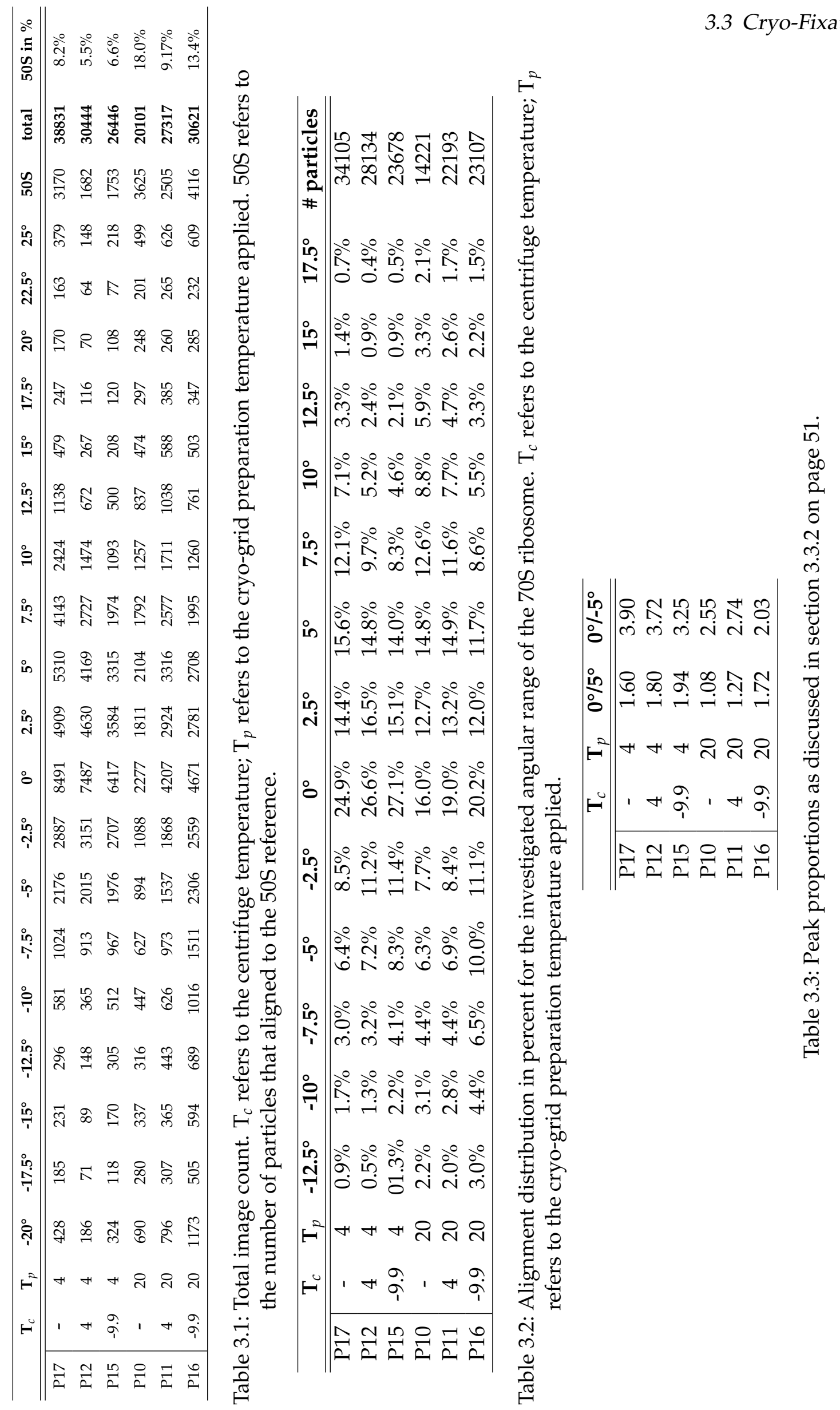




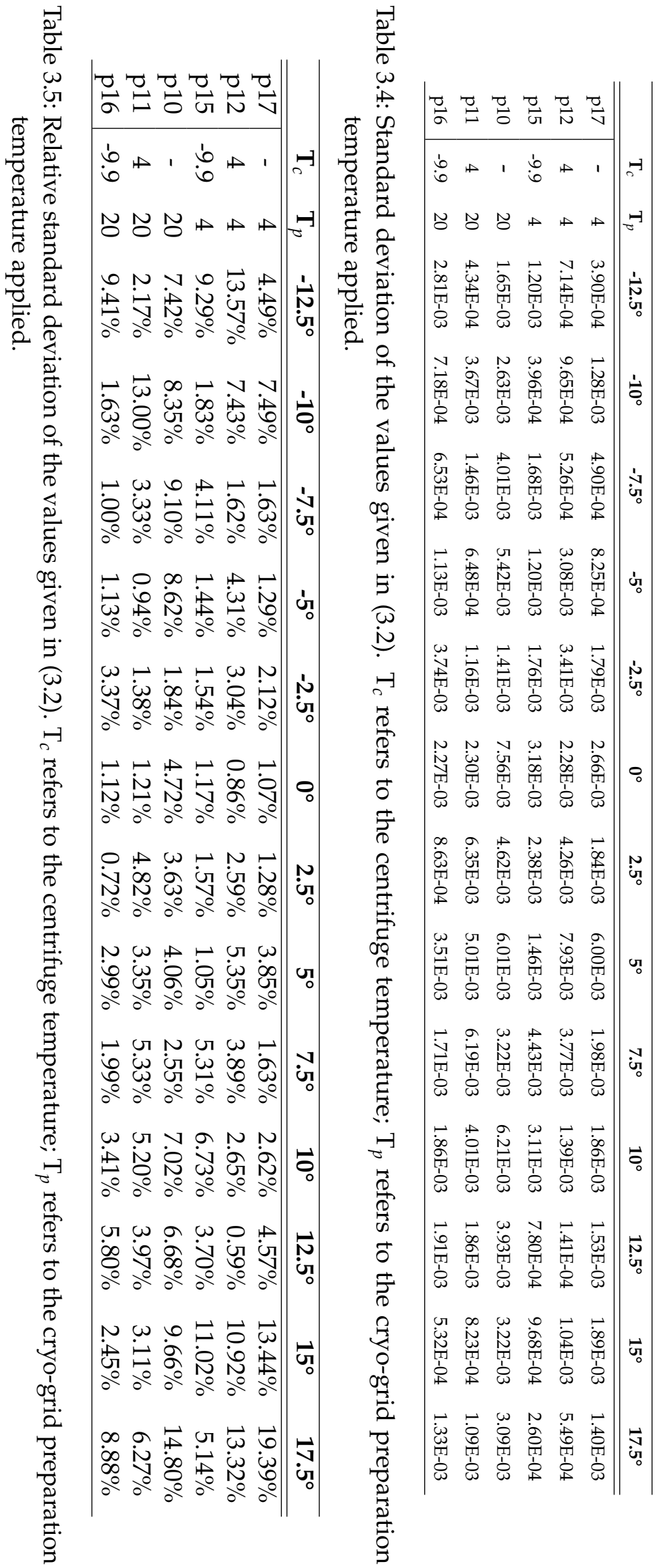




\section{Discussion}

\subsection{Spliceosomal remodeling EM-studies}

The spliceosome is a highly dynamic macromolecular complex. Not only does it undergo significant structural and compositional changes during the splicing cycle, but the intermediate complexes also possess conformational flexibility that induces further heterogeneity. One such rearrangement takes place during the catalytic activation of the $\mathrm{B}^{\text {act }}$ to the $\mathrm{B}^{*}$ spliceosomal complex [Kim and Lin, 1996, Fabrizio et al., 2009, Warkocki et al., 2009]. This rearrangement was examined with negative-stain electron microscopy on the 2D level to support the biochemical data and verify the assumed structural change. The structural difference was immediately visible in the class averages obtained from reference-free alignment. The validity of these differences was verified by injecting an artificial bias into the dataset. By aligning the images to a combined set of references obtained from the dominant views of both datasets, foreign structures were introduced. These caused some particles to align to the foreign references, resulting in corresponding class averages. This reference bias occurs mainly when only one or few different references are available [Boekema et al., 1986]. Since the alignment algorithm attempts to find the best possible match with the references, even random noise can be aligned and result in features of the original reference [Penczek et al., 1992]. The relatively small number of particles aligning to the foreign references already suggested that these observations were the result of model bias or - in the case of the sample after catalytic activation - structural heterogeneity caused by a non-quantitative conversion. Subsequent further alignment cycles supported this assumption by removing the foreign class averages from the $\mathrm{B}^{\text {act }}$-dataset while reducing them in the $\mathrm{B}^{*}$-dataset. The results are shown graphically in Fig. 3.2 on page 37. This shows that the initial class averages are indeed stable representatives of their respective dataset and verifies the structural rearrangement of the spliceosomal B-complex upon catalytic 


\section{Discussion}

activation. Furthermore, the presence of stable pre-activated class averages in the catalytically activated dataset corresponds with biochemical data that indicates a conversion rate of about 80-90\% [Warkocki et al., 2009].

\subsubsection{Outlook}

With the structural change confirmed and a good indication of it being quite significant, further research could focus on the visualization of this structural change and its intermediary steps on the 3D level. This would involve taking samples at various time points of the conversion reaction to sample a broad distribution of intermediary conformations, recording a large dataset and performing a hierarchical sorting analogous to Fischer et al. [2010]. High resolution structures of the starting and final configurations would be an essential first step in this project and could then be complemented by the transition structures. Structural diversity (i.e. heterogeneity) and high resolution are often at opposite ends of a spectrum, especially when the additional inner dynamic of the particles are considered. Controlling heterogeneity will be invaluable in facilitating such projects by allowing easier access to better resolved structures of defined states.

\subsection{Chemical modification}

The spliceosomal B-complex is a very dynamic particle that shows movement between a flexible head domain and a relatively rigid triangular body region. To account for the different orientations the head can adopt relative to the body, the dataset has to be subclassified into several substructures. This limits the resolution for three-dimensional reconstruction because each substructure only comprises a fraction of the images of the original dataset. To compensate for this loss in resolution, a correspondingly larger initial dataset is required. But with increased particle dynamics a disproportionately large number of images is needed. Limiting these dynamics would allow for fewer, higher resolved substructures from the same basic dataset. The significant improvement in sample quality gained by the application of the GraFix protocol [Kastner et al., 2008] suggests that further optimization on the preparation level is possible. This was pursued by modifying the fixation with secondary additives, namely: $\mathrm{Ta}_{6} \mathrm{Br}_{14}$ (experiment B), $\mathrm{K}_{2}\left[\mathrm{PtCl}_{6}\right](\mathrm{C}), \mathrm{Na}_{2} \mathrm{SO}_{4}(\mathrm{D})$, triflouroethanol (E), $\mathrm{CdCl}_{2}$ (F), PEG 4000 (G), PEG $8000(\mathrm{H})$. In experiment A, no additive was used. 


\subsubsection{Image level}

Examining the raw images and class averages, there was no immediately visible difference, apart from a higher contrast in the images of the class averages of condition $\mathrm{B}\left(\mathrm{Ta}_{6} \mathrm{Br}_{14}\right)$. This may be due to secondary staining effects from the additive, which is a relatively large heavy metal cluster [Knäblein et al., 1997]. The apparent effects on particle orientation and the occurrence of a dominant view were noted, but initially not further pursued. An objective method was required to assess the quality and homogeneity of the datasets beyond the visual inspection on the image level.

\subsubsection{FRC-analysis}

The ClassTest script and executable allowed a fast analysis of the image data. The premise was that a more homogeneous dataset would also produce more homogeneously composed class averages, which could be measured. The Imagic-file [van Heel et al., 1996] with the aligned particle images served as input and was classified, correlated and analyzed. The results for unaligned input images are predictably heterogeneous but have been included in the graphs in Fig. 3.6 on page 42 for the sake of completeness. The quality of the datasets stabilized after about 3 to 4 cycles of alignment and classification. Among the tested conditions, the plot of the qualities over the number of alignments points to the unmodified GraFix protocol actually having the best measurable effect on the sample. The validation of the results with the second datasets for conditions $\mathrm{A}$ and $\mathrm{B}$ (unmodified and $\mathrm{Ta}_{6} \mathrm{Br}_{14}$ respectively) showed the differences in quality to be not significant (see Fig. 3.10 on page 45). Apparently, the added reagents had no effect on sample heterogeneity. The hope for a silver bullet was not fulfilled and a larger scale screening in this manner was deemed unfeasible with the methods at hand. Therefore this approach was discontinued and other modifications of the preparation protocol were examined - leading to the CryoFix protocol described in section 4.3 on the following page.

\subsubsection{Outlook}

Recent research in our lab has established a screening method for preparation buffers utilizing ThermoFluor ${ }^{\circledR}$-based differential scanning fluorimetry [Chari et al., 2012]. This method allows a large number of conditions to be tested in a short time for their effect on protein stability. The 


\section{Discussion}

approach could revitalize the research on chemical additives for sample preparation and the way they affect structural heterogeneity. While the thermofluor method itself cannot directly assess the effect on conformational heterogeneity, it could help to narrow the selection of additives to those that do not destabilize the sample. In this way a preselection could be made that obviates the need for a complete sample preparation and image analysis for each potential additive. Instead, promising additives could be identified and then be prepared with a suitable model system under optimized buffer conditions. Finally analysis with the ClassTest software would evaluate the homogeneity of the sample.

\subsection{Cryo-Fixation}

Biological samples are usually handled between $0{ }^{\circ} \mathrm{C}$ and $4{ }^{\circ} \mathrm{C}$ by keeping them on ice or handling them in cold-rooms in order to minimize biological activity. Sample preparation for electron microscopy is also carried out at these temperatures. Studies with the ribosome have shown that preparation at higher temperatures leads to more heterogeneous datasets [Fischer et al., 2010].

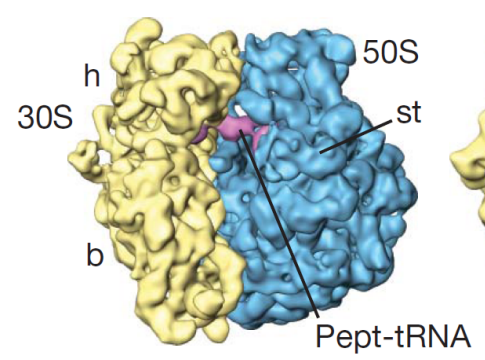

$4{ }^{\circ} \mathrm{C}$

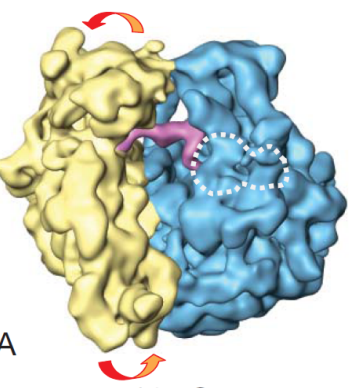

$18^{\circ} \mathrm{C}$

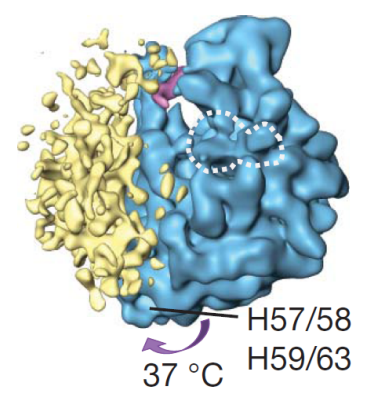

Figure 4.1: Three-dimensional reconstructions of ribosomes at different preparation temperatures. Increased temperatures facilitate movement (arrows), which negatively affects quality and detail of the reconstruction. The L12 stalk base (st) averages out at elevated temperatures (indicated by the dotted line) and the $30 \mathrm{~S}$ subunit only shows a scattered density due to strong heterogeneity. Image courtesy of Fischer et al. [2010]

Although the GraFix preparation does not completely eliminate heterogeneity, it does at least affect it. It was examined whether GraFix preparation at temperatures below $0{ }^{\circ} \mathrm{C}$ could limit heterogeneity in a desirable way by stabilizing the particles in a thermodynamically preferred conformation adopted at lowered temperatures. If the dynamics of the particles were not too strong, they could retain this preferred conformation even upon rewarming to $4{ }^{\circ} \mathrm{C}$, where the 
final preparation steps would take place. Conformational heterogeneity could be limited to fewer distinct substructures, thus reducing the number of images needed.

\subsubsection{The CryoFix protocol}

As has been stated in section 3.3 on page 46 , the set temperature of $-9.9^{\circ} \mathrm{C}$ could not be verified. Temperature measurements with an electronic thermometer inside the gradients immediately after centrifugation reached as low as $-6^{\circ} \mathrm{C}$. However, during this measurement the gradients were already warming rapidly since they had been removed from the centrifuge. It may be that the actual temperature during centrifugation was below the measured temperature. But even assuming the verified temperature of $-6^{\circ} \mathrm{C}$ results in a difference of $10^{\circ} \mathrm{C}$ compared to the usual $4{ }^{\circ} \mathrm{C}$. This is a significant change in temperature, which should favour a thermodynamically more stable conformation.

\section{Gradient Freezing}

In most cases, gradient freezing was not a problem. Apparently, the concentrations of buffer, salt and glycerol (or sucrose) in the gradients in combination with the pressure buildup that would result from the expansion upon freezing are strong enough to keep the gradients liquid. In those cases where gradients did freeze, it is assumed that freezing happened spontaneously upon opening of the bucket. The sudden pressure relaxation could lead to freezing of the super-cooled gradient from top to middle. This is supported by the observation that frozen gradients had the sample uniformly distributed in the lower, unfrozen half. Since this happened only occasionally, it was not considered critical to be further investigated. Letting gradients warm to $+4{ }^{\circ} \mathrm{C}$ before opening the buckets should prevent gradient freezing. Ensuring that the gradient surface does not come into contact with the metal of the bucket lid is also suggested (David Haselbach, personal communication).

\subsubsection{The ribosome as a model system}

With the technical requirements for the method fulfilled, a proof of principle had to be conducted. The intersubunit rotation of the $70 S$ ribosome provided a convenient indicator for heterogeneity. Furthermore, the library with structures of the various conformations [Fischer et al., 2010] 


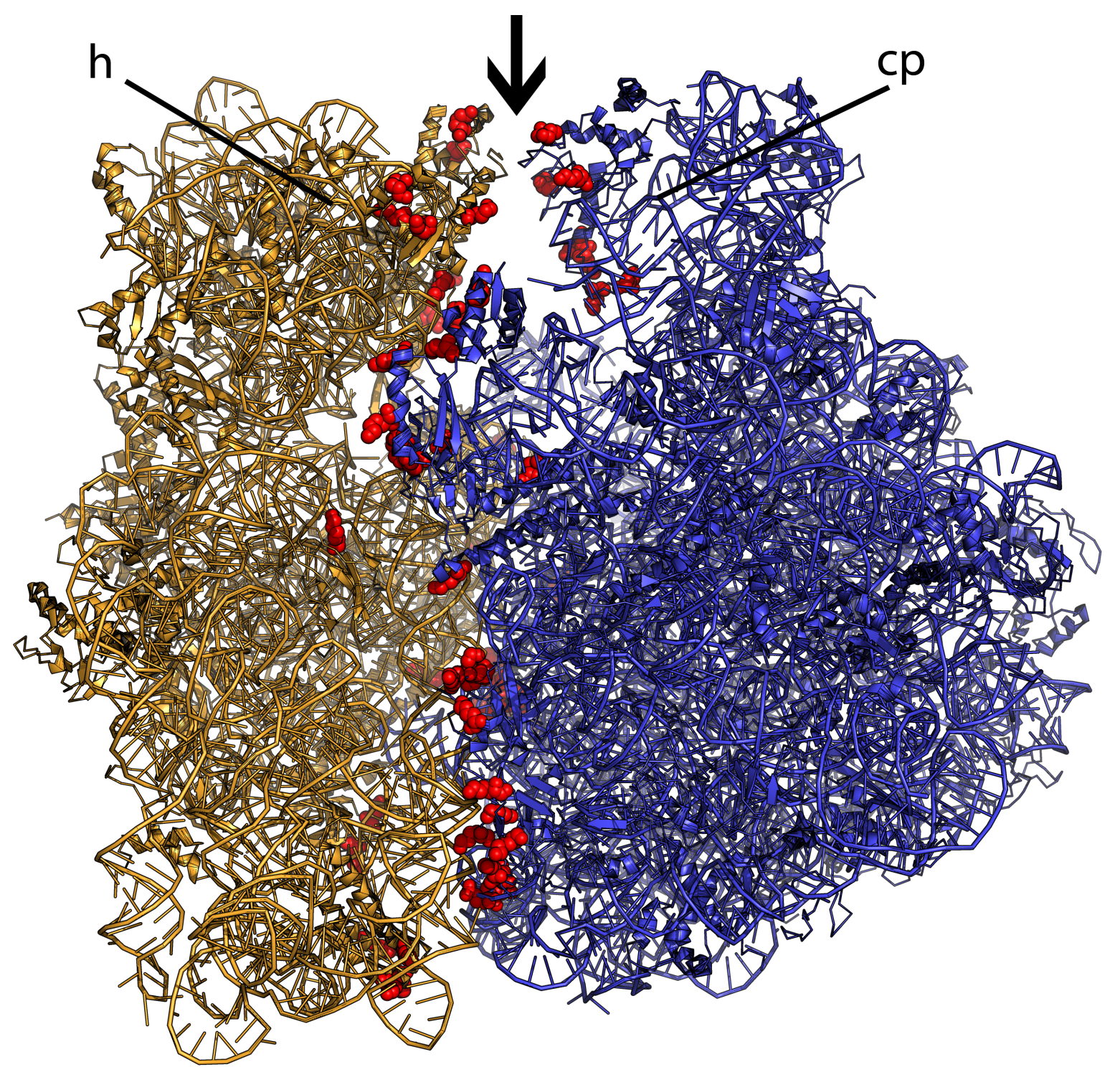

Figure 4.2: Cartoon model of the 70S ribosome with lysines near the contact area visualized as red spheres. The most significant crosslinking can only happen between the $30 \mathrm{~S}$ head domain (h) and the 50S central protuberance (cp) (see arrow). The lyines in the lower half of the image are located on the $50 \mathrm{~S}$ body and are faced by RNA on the $30 \mathrm{~S}$ side. Image generated from PDB entries 2QBD and 2QBE [Borovinskaya et al., 2007]. 
already existed in our lab and could be easily applied to the problem. However, with regard to glutaraldehyde fixation, the ribosome is a suboptimal model system. Large parts of its structure consist of RNA, which cannot be crosslinked by glutaraldehyde. This is especially true for the contact area between the subunits, where little protein structure is present. Glutaraldehyde possesses the highest crosslinking activity with lysine residues [Migneault et al., 2004, Bowes and Cater, 1968]. Therefore, the most significant crosslinking takes place between the $30 \mathrm{~S}$ head domain and the $50 \mathrm{~S}$ central protuberance, which both have lysine residues in proximity to each other (see Fig. 4.2 on the facing page). This means that even upon successful fixation, intersubunit movement will probably not be arrested, but merely constrained. Nonetheless, the advantages outweighed the limits of the system. Therefore the $70 \mathrm{~S}$ ribosome was chosen as a model system for the proof of principle. Since cryo-fixated samples cannot be prepared for cryoEM at negative temperatures, they will inevitably have to be warmed to ambient temperatures of $4{ }^{\circ} \mathrm{C}$. The premise for cryo-fixation to be an applicable method was therefore twofold: First, glutaraldehyde would have to stabilize a conformational state even upon subsequent warming. Secondly, the conformational distribution of a flexible macromolecule would have to narrow at the CryoFix temperature. The first requirement was investigated by preparing unfixated control samples that should show the full conformational range of their ambient preparation temperature. By also preparing samples at ambient temperatures of $20^{\circ} \mathrm{C}$ in the Vitrobot, the effect of fixation temperature on heterogeneity could be studied on a broader range. Since GraFix performs a mild fixation and this fixation is limited in extent on the ribosome, the stabilizing effect was not dramatic, as shown in Fig. 3.14 and 3.15 on page 52. Nonetheless, it is visible and significant, which proves that glutaraldehyde fixation can indeed stabilize the conformational distribution of a biological macromolecule. Even upon subsequent warming of the sample, this distribution is partially retained. Furthermore, cryo-fixation amplifies the effect of GraFix in the expected way.

\subsection{Conclusion and Outlook}

While in this work, the CryoFix protocol has only been tested on established systems, other projects in our lab have successfully applied it to other macromolecular complexes with promising results. For example, the preparation of snail hemoglobin BgHb [Lieb et al., 2006] benefits 


\section{Discussion}

significantly from cryo-fixation (Holger Stark, personal communication). CryoFix is a promising method that can further limit sample heterogeneity beyond the point of current preparation methods. Once the protocol has been adapted to a given sample with respect to centrifugation conditions and migration behaviour in the gradient, it entails no more work than regular gradient fixation. However, it might not be the ideal protocol for every sample and its effects may well vary. As aforementioned, the key to obtaining higher-resolution structures will lie in a synergy of preparative methods, state-of-the-art instruments, advanced computational methods and the processing power to implement them, but CryoFix is another step in the venture. 


\title{
Abbreviations
}

\author{
1D one-dimensional \\ 2D two-dimensional \\ 3D three-dimensional \\ ATP adenosine-5'-triphosphate \\ B* catalytically activated spliceosomal B-complex \\ $\mathrm{B}^{\text {act }} \quad$ activated spliceosomal B-complex \\ CCD charge coupled device \\ cryo-EM cryogenic electron microscopy \\ DTT dithiothreitol \\ $\mathrm{e}^{-} \quad$ electron(s) \\ E. coli Escherichia coli \\ EDTA ethylenediaminetetraacetic acid \\ e.g. exemplo gratia \\ EM electron microscopy \\ et al. et alii
}

Fig. $\quad$ Figure

FRC Fourier ring correlation

GDH glutamate dehydrogenase

GraFix gradient fixation

HEPES 4-(2-hydroxyethyl)-1-piperazineethanesulfonic acid

i.e. id est 
Abbreviations

MBP maltose binding protein

MPI-BPC Max-Planck institute for biophysical chemistry

MRA multi reference alignment

mRNA messenger-RNA

MSA multivariate statistical analysis

$\mathrm{NAD}(\mathrm{H}) \quad$ Nicotinamide adenine dinucleotide

NADP $(\mathrm{H})$ Nicotinamide adenine dinucleotide phosphate

OD optical density

PAGE polyacrylamide gel electrophoresis

PDB protein data bank

PEG poly ethylene glycol

Prp2 RNA-dependent ATPase, required for activation of the spliceosome before the first transesterification step in RNA splicing

RNA ribonucleic acid SNR signal-to-noise ratio

rpm rotations per minute

snRNA small nuclear ribonucleic acid

snRNP small nuclear ribonucleoproteins

TEM transmission electron microscope / microscopy

TEMED tetramethylethylenediamine

TFE trifluoroethanol

TRIS tris(hydroxymethyl)aminomethane

tRNA transfer-RNA

$[\mathrm{v} / \mathrm{v}] \quad$ volume per volume

[w/v] weight per volume 


\section{Bibliography}

M. Adrian, J. Dubochet, J. Lepault, and A. W. McDowall. Cryo-electron microscopy of viruses. Nature, 308(5954):32-36, 1984.

A. Ben-Shem, L. Jenner, G. Yusupova, and M. Yusupov. Crystal structure of the eukaryotic ribosome. Science, 330(6008):1203-1209, 2010.

E. J. Boekema, J. A. Berden, and M. G. van Heel. Structure of mitochondrial f1-atpase studied by electron microscopy and image processing. Biochim Biophys Acta, 851(3):353-360, 1986.

M. A. Borovinskaya, R. D. Pai, W. Zhang, B. S. Schuwirth, J. M. Holton, G. Hirokawa, H. Kaji, A. Kaji, and J. H D. Cate. Structural basis for aminoglycoside inhibition of bacterial ribosome recycling. Nat Struct Mol Biol, 14(8):727-732, 2007.

J. H. Bowes and C. W. Cater. The interaction of aldehydes with collagen. Biochim Biophys Acta, 168(2):341-352, 1968.

P. Brüggeller and E. Mayer. Complete vitrification on pure liquid water and dilute aqueous solutions. Nature, 288:569-571, 1980.

A. Chari, D. Haselbach, Ohmer J., E. Paknia, J.-M. Kirves, U. Steuerwald, N. Fischer, O. Ganichkin, U. Fischer, V. Pena, and H. Stark. A sample optimization workflow for structural studies of macromolecular complexes. Manuscript in preparation, 2012.

Patrizia Fabrizio, Julia Dannenberg, Prakash Dube, Berthold Kastner, Holger Stark, Henning Urlaub, and Reinhard Lührmann. The evolutionarily conserved core design of the catalytic activation step of the yeast spliceosome. Mol Cell, 36(4):593-608, 2009.

N. Fischer, A. L. Konevega, W. Wintermeyer, M. V. Rodnina, and H. Stark. Ribosome dynamics and tRNA movement by time-resolved electron cryomicroscopy. Nature, 466(7304):329-333, 2010.

H. Gao, M. Valle, M. Ehrenberg, and J. Frank. Dynamics of ef-g interaction with the ribosome 


\section{Bibliography}

explored by classification of a heterogeneous cryo-em dataset. J Struct Biol, 147(3):283-290, 2004.

M.A. Hayat. Glutaraldehyde: Role in electron microscopy. Micron and Microscopica Acta, 17(2): 115 - 135, 1986. ISSN 0739-6260.

R. Henderson. The potential and limitations of neutrons, electrons and x-rays for atomic resolution microscopy of unstained biological molecules. Q. Rev. Biophys., 28:171-193, 1995.

L. H. Horan and H. F. Noller. Intersubunit movement is required for ribosomal translocation. Proc Natl Acad Sci U S A, 104(12):4881-4885, 2007.

A. A. Infante and R. Baierlein. Pressure-induced dissociation of sedimenting ribosomes: effect on sedimentation patterns. Proc Natl Acad Sci U S A, 68(8):1780-1785, 1971.

B. Kastner, N. Fischer, M. M. Golas, B. Sander, P. Dube, D. Boehringer, K. Hartmuth, J. Deckert, F. Hauer, E. Wolf, H. Uchtenhagen, H. Urlaub, F. Herzog, J. M. Peters, D. Poerschke, R. Luhrmann, and H. Stark. Grafix: sample preparation for single-particle electron cryomicroscopy. Nat Methods, 5(1):53-55, 2008.

J. Kawahara, T. Ohmori, T. Ohkubo, S. Hattori, and M. Kawamura. The structure of glutaraldehyde in aqueous solution determined by ultraviolet absorption and light scattering. Anal Biochem, 201(1):94-98, 1992.

S. H. Kim and R. J. Lin. Spliceosome activation by prp2 atpase prior to the first transesterification reaction of pre-mrna splicing. Mol Cell Biol, 16(12):6810-6819, 1996.

J. Knäblein, T. Neuefeind, F. Schneider, A. Bergner, A. Messerschmidt, J. Löwe, B. Steipe, and R. Huber. $\mathrm{Ta} 6 \mathrm{Br}(2+) 12$, a tool for phase determination of large biological assemblies by $\mathrm{x}$-ray crystallography. J Mol Biol, 270(1):1-7, 1997.

Steven B. Larson, John S. Day, Robert Cudney, and Alexander McPherson. A novel strategy for the crystallization of proteins: X-ray diffraction validation. Acta Crystallogr D Biol Crystallogr, 63(Pt 3):310-318, 2007.

D. M. Lawson, P. J. Artymiuk, S. J. Yewdall, J. M. Smith, J. C. Livingstone, A. Treffry, A. Luzzago, S. Levi, P. Arosio, and G. Cesareni. Solving the structure of human h ferritin by genetically engineering intermolecular crystal contacts. Nature, 349(6309):541-544, 1991.

R. Leberman. Use of uranyl formate as a negative stain. J Mol Biol, 13(2):606, 1965. 
A. E. Leschziner and E. Nogales. Visualizing flexibility at molecular resolution: Analysis of heterogeneity in single-particle electron microscopy reconstructions. Annual Review of Biophysics and Biomolecular Structure, 36(1):43-62, 2007.

R. Lührmann and H. Stark. Structural mapping of spliceosomes by electron microscopy. Curr Opin Struct Biol, 19(1):96-102, 2009.

B. Lieb, K. Dimitrova, H.-S. Kang, S. Braun, W. Gebauer, A. Martin, B. Hanelt, S. A. Saenz, C. M. Adema, and J. Markl. Red blood with blue-blood ancestry: intriguing structure of a snail hemoglobin. Proc Natl Acad Sci U S A, 103(32):12011-12016, 2006.

S. J. Ludtke, P. R. Baldwin, and W. Chiu. Eman: semiautomated software for high-resolution single-particle reconstructions. J Struct Biol, 128(1):82-97, 1999.

A. McPherson. Use of polyethylene glycol in the crystallization of macromolecules. Methods Enzymol, 114:120-125, 1985.

A. McPherson and B. Cudney. Searching for silver bullets: an alternative strategy for crystallizing macromolecules. J Struct Biol, 156(3):387-406, 2006.

I. Migneault, C. Dartiguenave, M. J. Bertrand, and K. C. Waldron. Glutaraldehyde: behavior in aqueous solution, reaction with proteins, and application to enzyme crosslinking. Biotechniques, 37(5):790-6, 798-802, 2004.

P. Penczek, M. Radermacher, and J. Frank. Three-dimensional reconstruction of single particles embedded in ice. Ultramicroscopy, 40(1):33-53, 1992.

P. A. Penczek, R. A. Grassucci, and J. Frank. The ribosome at improved resolution: new techniques for merging and orientation refinement in $3 \mathrm{~d}$ cryo-electron microscopy of biological particles. Ultramicroscopy, 53(3):251-270, 1994.

P. E. Peterson and T. J. Smith. The structure of bovine glutamate dehydrogenase provides insights into the mechanism of allostery. Structure, 7(7):769-782, 1999.

Daniel A. Pomeranz Krummel, Chris Oubridge, Adelaine K W. Leung, Jade Li, and Kiyoshi Nagai. Crystal structure of human spliceosomal u1 snrnp at 5.5 a resolution. Nature, 458 (7237):475-480, 2009.

Carlos H I. Ramos and Robert L. Baldwin. Sulfate anion stabilization of native ribonuclease a both by anion binding and by the hofmeister effect. Protein Sci, 11(7):1771-1778, 2002. 


\section{Bibliography}

L Reimer. Transmission electron microscopy; physics of image formation and microanalysis. Springer series in optical sciences; Vol. 36. Springer Verlag, 4 edition, 1997. ISBN 3-540-62568-2.

B. Sander, M. M. Golas, and H. Stark. Automatic ctf correction for single particles based upon multivariate statistical analysis of individual power spectra. J Struct Biol, 142(3):392-401, 2003a.

B. Sander, M. M. Golas, and H. Stark. Corrim-based alignment for improved speed in singleparticle image processing. J Struct Biol, 143(3):219-228, 2003b.

T Martin Schmeing and V. Ramakrishnan. What recent ribosome structures have revealed about the mechanism of translation. Nature, 461(7268):1234-1242, 2009.

K. Shiraki, K. Nishikawa, and Y. Goto. Trifluoroethanol-induced stabilization of the alpha-helical structure of beta-lactoglobulin: implication for non-hierarchical protein folding. J Mol Biol, 245(2):180-194, 1995.

J. M. Sperrazza, D. W. Russell, and L. L. Spremulli. Reversible dissociation of wheat germ ribosomal subunits: cation-dependent equilibria and thermodynamic parameters. Biochemistry, 19 (6):1053-1058, 1980.

J. P. Staley and C. Guthrie. Mechanical devices of the spliceosome: motors, clocks, springs, and things. Cell, 92(3):315-326, 1998.

H. Stark, P. Dube, R. Lührmann, and B. Kastner. Arrangement of rna and proteins in the spliceosomal u1 small nuclear ribonucleoprotein particle. Nature, 409(6819):539-542, 2001.

M. van Heel. Angular reconstitution: a posteriori assignment of projection directions for 3d reconstruction. Ultramicroscopy, 21:111-124, 1987a.

M. van Heel and J. Frank. Use of multivariate statistics in analysing the images of biological macromolecules. Ultramicroscopy, 6(2):187-194, 1981.

M. van Heel, G. Harauz, E. V. Orlova, R. Schmidt, and M. Schatz. A new generation of the imagic image processing system. J Struct Biol, 116(1):17-24, 1996.

Marin van Heel. Multivariate statistical classification of noisy images (randomly oriented biological macromolecules). Ultramicroscopy, 13:165-184, 1984.

Marin van Heel. Similarity measures between images. Ultramicroscopy, 21(1):95 - 100, 1987b.

Z. Warkocki, P. Odenwälder, J. Schmitzová, F. Platzmann, H. Stark, H. Urlaub, R. Ficner, P. Fab- 
rizio, and R. Lührmann. Reconstitution of both steps of saccharomyces cerevisiae splicing with purified spliceosomal components. Nat Struct Mol Biol, 16(12):1237-1243, 2009.

Gert Weber, Simon Trowitzsch, Berthold Kastner, Reinhard Lührmann, and Markus C. Wahl. Functional organization of the Sm core in the crystal structure of human U1 snRNP. EMBO J, 29(24):4172-4184, 2010.

C. L. Will and R. Lührmann. Spliceosome structure and function. Cold Spring Harb Perspect Biol, 3(7):1, 2011.

A. Yonath, J. Harms, H. A. Hansen, A. Bashan, F. Schlünzen, I. Levin, I. Koelln, A. Tocilj, I. Agmon, M. Peretz, H. Bartels, W. S. Bennett, S. Krumbholz, D. Janell, S. Weinstein, T. Auerbach, H. Avila, M. Piolleti, S. Morlang, and F. Franceschi. Crystallographic studies on the ribosome, a large macromolecular assembly exhibiting severe nonisomorphism, extreme beam sensitivity and no internal symmetry. Acta Crystallogr A, 54(Pt 6 Pt 1):945-955, 1998.

W. Zhang, Jack A. Dunkle, and Jamie H D. Cate. Structures of the ribosome in intermediate states of ratcheting. Science, 325(5943):1014-1017, 2009. 



\section{Acknowledgements}

I would like to express my deepest gratitude to Prof. Holger Stark, whose enthusiasm for science in general and the method of three-dimensional electron microscopy in particular was always an example for me. Thank you for your supervision and your support and thank you for introducing me to the fascinating world of electron microscopy.

I wish to thank the members of my thesis committee, Prof. Reinhard Lührmann and Prof. Ralf Ficner, for their guidance and their advice. I am also grateful for the help from and discussions with my collaborators, Dr. Elmar Wolf, Zbigniew Warkocki and Ulrich Steuerwald.

Particularly, I wish to thank Dr. Niels Fischer, without whose support and guidance this work would not have been possible. Special thanks goes to Dr. Florian Hauer, who reassured me more than once in moments of doubt and whose pragmatism helped me remember that most problems are not as big as they may first seem. To them and all the other members of the cryo-EM group - especially my office colleagues, Wen-Ti Liu, Andrius Krasauskas and David Haselbach - I express my gratitude for their company and the good times and meals we shared. I also want to thank Dr. Prakash Dube, who is the good soul of the group, even though he tries to hide this (unsuccessfully) behind the facade of a grumpy old man.

Furthermore, I also wish to gratefully mention the GGNB coordination and administration, especially Dr. Steffen Burkhardt, Kirsten Pöhlker and Christina Bach, whose endeavours have created this outstanding studying environment I had the fortune to benefit from.

I am indebted to my parents, who always supported me without question and to whom this work is dedicated.

And finally, I wish to thank my wife, Andrea Thorn, for her patience, her motivation and her love.

I am truly standing on the shoulders of giants. 